\title{
Designing force probes based on reversible $6 \pi$-electrocyclizations in polyenes using quantum chemical calculations
}

\author{
Tom Bettens, ${ }^{[a]}$ Jochen Eeckhoudt, ${ }^{[a]}$ Marvin Hoffmann, ${ }^{[b]}$ Mercedes Alonso, ${ }^{[a]}$ Paul \\ Geerlings, ${ }^{[a]}$ Andreas Dreuw, ${ }^{[*, b]}$ Frank De Proft ${ }^{[*, a]}$

\begin{abstract}
[a] Eenheid Algemene Chemie (ALGC), Vrije Universiteit Brussel (VUB), Pleinlaan 2, 1050 Brussels, Belgium ${ }^{[b]}$ Interdisciplinary Center for Scientific Computing, Ruprecht-Karls University, Im Neuenheimer Feld 205A, D69120 Heidelberg, Germany
\end{abstract}

\section{Contents}

Figure S1. The $6 \pi$ electrocyclic ring-closure reactions $\left(R_{1}-R_{4}\right)$ used for the benchmarking study.

Table S1. Electronic reaction energies corresponding to the $6 \pi$ electrocyclic ring-closure reactions in Figure S1. All geometries were optimized using the B3LYP-D3BJ functional and DLPNO-CCSD(T) energies were used as reference. All energies are in $\mathrm{kcal} \mathrm{mol}^{-1}$.

Table S2. Electronic reaction energies $\left(\Delta_{r} E\right)$ and transition state energies $\left(\Delta E^{\ddagger}\right)$ of the $6 \pi$ electrocyclic ring-closures (under zero-force conditions) and ring-openings (when a force of 2 $\mathrm{nN}$ is used). All values are in $\mathrm{kcal} \mathrm{mol}^{-1}$.

Table S3. Reaction enthalpies $\left(\Delta_{r} H\right)$ and transition state enthalpies $\left(\Delta H^{\ddagger}\right)$ of the $6 \pi$ electrocyclic ring-closures (under zero-force conditions) and ring-openings (when a force of 2 $\mathrm{nN}$ is used). All values are in $\mathrm{kcal} \mathrm{mol}^{-1}$.....

Figure S2. Bond angles (in ${ }^{\circ}$ ) around $\mathrm{C}^{1}$.....

Figure S3. Bond angles (in ${ }^{\circ}$ ) around $\mathrm{C}^{2}$. .57

Figure S4. Bond angles (in ${ }^{\circ}$ ) around $\mathrm{C}^{3}$. .58

Figure S5. Dihedral angle inversion in 5'A owing to the application of an external force of 2 $\mathrm{nN}$.

Figure S6. Distribution of stress energy in $\mathbf{1 A}, \mathbf{2 A}$ and $\mathbf{5}^{\prime} \mathbf{B}\left(\underline{F}_{\text {ext }}=2 \mathrm{nN}\right)$, and the fraction of stress energy in bonds, angles and dihedral angles. The pulling positions are indicated in blue and distances are in $\AA$.

Table S4. Cartesian coordinates of each structure optimized at the wB97X-D3/Def2-TZ2P level of theory. The applied force, point group symmetry, electronic energy ( $E$ in a.u.), total enthalpy ( $H$ in a.u.), Gibbs energy ( $G$ in a.u.), number of imaginary frequencies $\left(n_{\text {imag }}\right)$ and imaginary vibrational frequency (for transitions states) are indicated. S10 
<smiles>[CH]1C[C@H]2C[C@@H]3C[C@H]4C=CC=C4C3=C2C2=CC=C[C@@H]12</smiles><smiles>[R7][CH-]C</smiles>

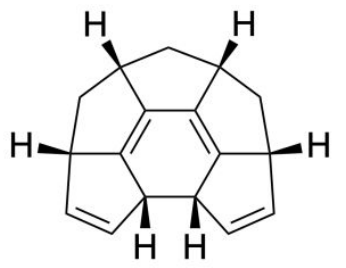<smiles>[CH]1C[C@H]2C=C[C@@H]3C[C@H]4C=CC=C4C3=C2C2=CC=C[C@@H]12</smiles><smiles>[R][CH]C</smiles>

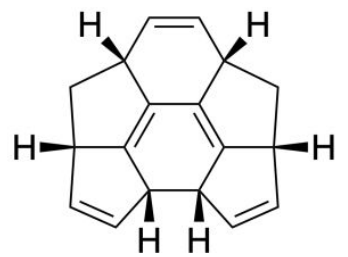<smiles></smiles><smiles>[R3][CH]C</smiles>

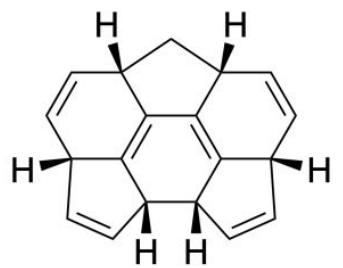<smiles></smiles>

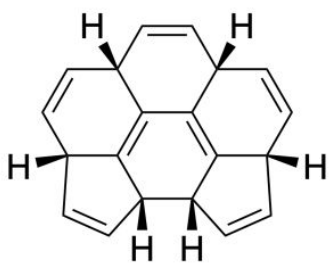

Figure S1. The $6 \pi$ electrocyclic ring-closure reactions $\left(R_{1}-R_{4}\right)$ used for the benchmarking study. 
Table S1. Electronic reaction energies corresponding to the $6 \pi$ electrocyclic ring-closure reactions in Figure S1. All geometries were optimized using the B3LYP-D3BJ functional and DLPNO-CCSD(T) energies were used as reference. All energies are in $\mathrm{kcal} \mathrm{mol}^{-1}$.

\begin{tabular}{|c|c|c|c|c|c|c|c|c|c|c|}
\hline & \multicolumn{2}{|c|}{$\Delta_{\mathrm{r}} E\left(\mathrm{R}_{1}\right)$} & \multicolumn{2}{|c|}{$\Delta_{r} E\left(R_{2}\right)$} & \multicolumn{2}{|c|}{$\Delta_{\mathrm{r}} E\left(\mathrm{R}_{3}\right)$} & \multicolumn{2}{|c|}{$\Delta_{\mathrm{r}} E\left(\mathrm{R}_{4}\right)$} & \multirow[t]{2}{*}{ MUE } & \multirow[t]{2}{*}{ RMSD } \\
\hline & $0 \mathrm{nN}$ & $2 \mathrm{nN}$ & $0 \mathrm{nN}$ & $2 \mathrm{nN}$ & $0 \mathrm{nN}$ & $2 \mathrm{nN}$ & $0 \mathrm{nN}$ & $2 \mathrm{nN}$ & & \\
\hline DLPNO-CCSD(T) & 5.3 & 102.4 & -0.6 & 84.5 & -8.7 & 72.0 & -12.1 & 51.9 & - & - \\
\hline B3LYP & 16.6 & 110.9 & 9.5 & 92.6 & 1.1 & 80.1 & -4.9 & 58.9 & 8.7 & 8.9 \\
\hline B3LYP-D3BJ & 12.4 & 107.5 & 6.4 & 90.2 & -1.7 & 77.9 & -6.6 & 57.2 & 6.1 & 6.1 \\
\hline B2PLYP & 13.0 & 108.4 & 6.4 & 90.3 & -1.8 & 77.8 & -6.7 & 57.2 & 6.3 & 6.3 \\
\hline CAM-B3LYP & 10.3 & 106.1 & 3.6 & 87.7 & -5.5 & 74.2 & -10.8 & 53.0 & 3.0 & 3.3 \\
\hline M06 & 10.4 & 107.1 & 4.5 & 88.5 & -3.7 & 75.9 & -9.0 & 54.1 & 4.1 & 4.2 \\
\hline M06-2X & 8.1 & 104.9 & 2.0 & 86.8 & -6.7 & 73.4 & -10.6 & 52.7 & 2.0 & 2.1 \\
\hline PBE & 14.9 & 109.0 & 8.1 & 90.8 & 0.6 & 79.2 & -5.3 & 58.4 & 7.6 & 7.7 \\
\hline wB97X-D3 & 5.6 & 102.0 & -0.7 & 84.0 & -9.4 & 70.5 & -13.7 & 49.9 & 0.9 & 1.1 \\
\hline wB97X-D3BJ & 3.1 & 100.7 & -2.7 & 83.0 & -11.5 & 69.3 & -15.4 & 48.6 & 2.5 & 2.5 \\
\hline
\end{tabular}


Table S2. Electronic reaction energies $\left(\Delta_{\mathrm{r}} E\right)$ and transition state energies $\left(\Delta E^{\ddagger}\right)$ of the $6 \pi$ electrocyclic ring-closures (under zero-force conditions) and ring-openings (when a force of 2 $\mathrm{nN}$ is used). All values are in $\mathrm{kcal} \mathrm{mol}^{-1}$.

\begin{tabular}{|c|c|c|c|c|c|c|c|c|c|c|c|}
\hline \multirow[t]{2}{*}{$\begin{array}{c}\text { Struc- } \\
\text { ture } \\
\end{array}$} & \multirow[t]{2}{*}{ Path } & \multicolumn{2}{|c|}{$\begin{array}{l}\text { Forward } \\
(0 \mathrm{nN})\end{array}$} & \multicolumn{2}{|c|}{$\begin{array}{l}\text { Reverse } \\
(2 \mathrm{nN})\end{array}$} & \multirow[t]{2}{*}{$\begin{array}{l}\text { Struc- } \\
\text { ture }\end{array}$} & \multirow[t]{2}{*}{ Path } & \multicolumn{2}{|c|}{$\begin{array}{l}\text { Forward } \\
(0 \mathrm{nN})\end{array}$} & \multicolumn{2}{|c|}{$\begin{array}{l}\text { Reverse } \\
(2 \mathrm{nN})\end{array}$} \\
\hline & & $\Delta E^{\ddagger}$ & $\Delta_{\mathrm{r}} E$ & $\Delta E^{\ddagger}$ & $\Delta_{\mathrm{r}} E$ & & & $\Delta E^{\ddagger}$ & $\Delta_{\mathrm{r}} E$ & $\Delta E^{\ddagger}$ & $\Delta_{\mathrm{r}} E$ \\
\hline \multirow[t]{2}{*}{1} & A & 26.1 & -9.0 & 12.6 & -70.8 & $1^{\prime}$ & $\mathrm{A}^{[\mathrm{a}]}$ & 48.5 & 41.6 & 0.2 & -102.5 \\
\hline & B & 63.4 & 50.8 & 1.3 & -104.8 & & B & 71.4 & 27.8 & 15.1 & -95.6 \\
\hline \multirow[t]{2}{*}{2} & A & 23.8 & -7.9 & 15.6 & -72.0 & $2^{\prime}$ & A & 46.6 & 37.3 & 0.3 & -98.3 \\
\hline & B & 52.8 & 47.2 & 0.4 & -100.7 & & B & 57.9 & 18.8 & 14.0 & -83.5 \\
\hline \multirow[t]{2}{*}{3} & A & 26.8 & -4.3 & 15.7 & -65.3 & $3^{\prime}$ & A & 30.9 & 12.1 & 7.8 & -71.0 \\
\hline & B & 28.9 & 6.9 & 9.2 & -68.2 & & B & 43.8 & -3.3 & 23.1 & -57.2 \\
\hline \multirow[t]{2}{*}{4} & A & 22.1 & -8.1 & 14.3 & -67.7 & $4^{\prime}$ & A & 36.0 & 16.9 & 6.0 & -74.6 \\
\hline & B & 27.3 & 8.3 & 7.2 & -72.7 & & B & 40.5 & -3.4 & 19.8 & -58.9 \\
\hline \multirow[t]{2}{*}{5} & A & 26.6 & -4.5 & 13.6 & -61.5 & $5^{\prime}$ & A & 24.8 & 3.6 & 7.7 & -61.7 \\
\hline & B & 26.2 & 2.7 & 9.2 & -64.4 & & B & 32.9 & -10.0 & 19.3 & -49.7 \\
\hline
\end{tabular}

[a] The reverse path was calculated at $1.8 \mathrm{nN}$ because the closed structure is no longer a minimum on the PES at $2 \mathrm{nN}$. 
Table S3. Reaction enthalpies $\left(\Delta_{\mathrm{r}} H\right)$ and transition state enthalpies $\left(\Delta H^{\ddagger}\right)$ of the $6 \pi$ electrocyclic ring-closures (under zero-force conditions) and ring-openings (when a force of 2 $\mathrm{nN}$ is used). All values are in $\mathrm{kcal} \mathrm{mol}^{-1}$.

\begin{tabular}{|c|c|c|c|c|c|c|c|c|c|c|c|}
\hline \multirow[t]{2}{*}{$\begin{array}{l}\text { Struc- } \\
\text { ture } \\
\end{array}$} & \multirow[t]{2}{*}{ Path } & \multicolumn{2}{|c|}{$\begin{array}{l}\text { Forward } \\
(0 \mathrm{nN})\end{array}$} & \multicolumn{2}{|c|}{$\begin{array}{l}\text { Reverse } \\
(2 \mathrm{nN})\end{array}$} & \multirow[t]{2}{*}{$\begin{array}{c}\text { Struc- } \\
\text { ture }\end{array}$} & \multirow[t]{2}{*}{ Path } & \multicolumn{2}{|c|}{$\begin{array}{l}\text { Forward } \\
(0 \mathrm{nN})\end{array}$} & \multicolumn{2}{|c|}{$\begin{array}{l}\text { Reverse } \\
(2 \mathrm{nN})\end{array}$} \\
\hline & & $\Delta H^{\ddagger}$ & $\Delta_{\mathrm{r}} H$ & $\Delta H^{\ddagger}$ & $\Delta_{\mathrm{r}} \mathrm{H}$ & & & $\Delta H^{\ddagger}$ & $\Delta_{\mathrm{r}} \mathrm{H}$ & $\Delta H^{\ddagger}$ & $\Delta_{\mathrm{r}} \mathrm{H}$ \\
\hline \multirow[t]{2}{*}{1} & $A$ & 25.4 & -7.5 & 10.9 & -72.4 & $1^{\prime}$ & $\mathrm{A}^{[\mathrm{a}]}$ & 47.8 & 42.3 & 0.0 & -103.4 \\
\hline & B & 62.5 & 51.5 & 0.3 & -105.6 & & B & 70.2 & 28.8 & 13.4 & -96.6 \\
\hline \multirow[t]{2}{*}{2} & A & 22.9 & -6.9 & 14.1 & -73.0 & $2^{\prime}$ & A & 46.0 & 38.0 & 0.0 & -99.1 \\
\hline & B & 51.9 & 47.5 & -0.5 & -101.1 & & B & 56.9 & 19.7 & 12.4 & -84.4 \\
\hline \multirow[t]{2}{*}{3} & $A$ & 26.2 & -3.2 & 14.1 & -66.4 & $3^{\prime}$ & A & 30.3 & 13.1 & 6.3 & -72.0 \\
\hline & B & 28.1 & 7.8 & 7.8 & -69.0 & & B & 42.7 & -2.5 & 19.5 & -58.2 \\
\hline \multirow[t]{2}{*}{4} & A & 21.2 & -7.1 & 12.6 & -68.9 & $4^{\prime}$ & A & 35.4 & 17.8 & 4.7 & -75.6 \\
\hline & B & 26.3 & 8.8 & 5.9 & -73.4 & & B & 39.6 & -2.6 & 18.1 & -59.9 \\
\hline \multirow[t]{2}{*}{5} & A & 25.8 & -3.7 & 12.2 & -62.3 & $5^{\prime}$ & A & 24.2 & 4.7 & 6.4 & -62.6 \\
\hline & B & 25.0 & 3.4 & 7.8 & -65.0 & & B & 31.8 & -9.2 & 17.5 & -50.6 \\
\hline
\end{tabular}

[a] The reverse path was calculated at $1.8 \mathrm{nN}$ because the closed structure is no longer a minimum on the PES at $2 \mathrm{nN}$. 

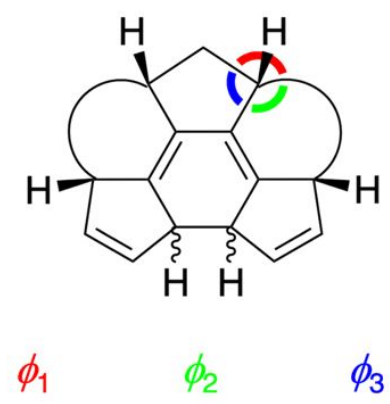

$\begin{array}{llll}1 \mathrm{~A} & 114.8 & 111.1 & 102.3\end{array}$

$\begin{array}{llll}\text { 1B } & 119.4 & 106.9 & 99.2\end{array}$

$\begin{array}{llll}2 A & 117.6 & 101.5 & 112.6\end{array}$

$\begin{array}{llll}\text { 2B } & 121.7 & 108.6 & 98.8\end{array}$

$\begin{array}{llll}3 A & 113.3 & 121.1 & 103.1\end{array}$

$\begin{array}{llll}3 B & 117.6 & 113.9 & 101.1\end{array}$

$\begin{array}{llll}\text { 4A } & 116.5 & 120.1 & 102.8\end{array}$

4B $\quad 115.1 \quad 113.1 \quad 101.2$

$\begin{array}{llll}\mathbf{5 A} & 110.8 & 118.8 & 104.0\end{array}$

$\begin{array}{llll}5 B & 116.2 & 112.3 & 101.6\end{array}$

Figure S2. Bond angles (in ${ }^{\circ}$ ) around $\mathrm{C}^{1}$.

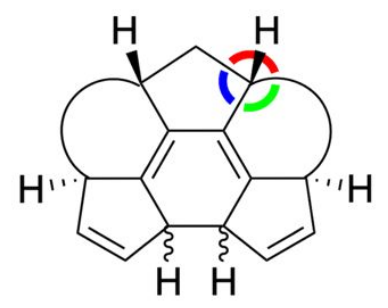

$\begin{array}{lll}\phi_{1} & \phi_{2} & \phi_{3}\end{array}$

$\begin{array}{llll}1 ' A & 120.7 & 111.8 & 101.4\end{array}$

$\begin{array}{llll}1 ' B & 132.6 & 109.6 & 101.7\end{array}$

$\begin{array}{llll}\text { 2'A } & 117.3 & 112.7 & 101.7\end{array}$

$\begin{array}{llll}\text { 2'B } & 129.8 & 107.5 & 100.8\end{array}$

$\begin{array}{llll}\text { 3'A } & 117.3 & 121.0 & 102.5\end{array}$

$\begin{array}{llll}\text { 3'B } & 118.5 & 115.1 & 102.1\end{array}$

$\begin{array}{llll}\text { 4'A } & 112.6 & 117.8 & 102.4\end{array}$

$\begin{array}{llll}\text { 4'B } & 115.7 & 115.3 & 102.2\end{array}$

$\begin{array}{llll}\text { 5'A } & 118.1 & 116.5 & 102.5\end{array}$

$\begin{array}{llll}\text { 5'B } & 117.9 & 112.7 & 101.4\end{array}$ 

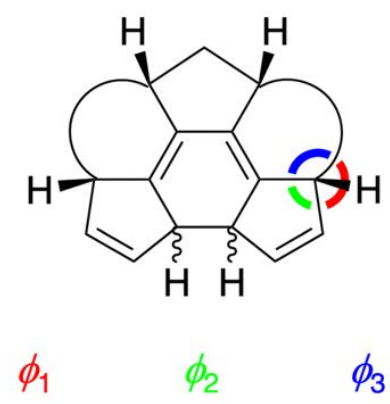

$\begin{array}{llll}\text { 1A } & 117.0 & 99.9 & 112.0\end{array}$

$\begin{array}{llll}\text { 1B } & 127.8 & 100.7 & 104.7\end{array}$

$\begin{array}{llll}2 A & 115.0 & 100.5 & 109.5\end{array}$

$\begin{array}{llll}\text { 2B } & 126.3 & 99.8 & 103.4\end{array}$

$\begin{array}{llll}\text { 3A } & 111.5 & 101.2 & 114.1\end{array}$

$\begin{array}{llll}\text { 3B } & 120.6 & 100.7 & 111.1\end{array}$

$\begin{array}{llll}\text { 4A } & 111.7 & 101.1 & 112.5\end{array}$

$\begin{array}{llll}\text { 4B } & 120.9 & 100.5 & 110.7\end{array}$

$\begin{array}{llll}\text { 5A } & 112.9 & 100.7 & 118.5\end{array}$

$\begin{array}{llll}\text { 5B } & 120.9 & 101.1 & 112.5\end{array}$

Figure S3. Bond angles (in ${ }^{\circ}$ ) around $\mathrm{C}^{2}$.

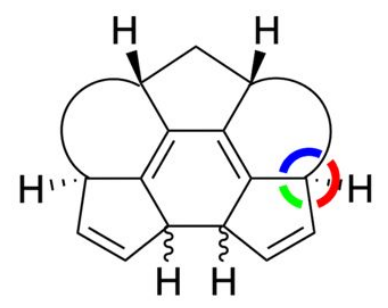

$\phi_{1} \quad \phi_{2} \quad \phi_{3}$

$\begin{array}{llll}\text { 1'A } & 132.3 & 102.1 & 111.0\end{array}$

$\begin{array}{llll}1 \text { 1'B } & 124.5 & 96.6 & 111.4\end{array}$

$\begin{array}{llll}\text { 2'A } & 131.5 & 101.6 & 108.9\end{array}$

$\begin{array}{llll}\text { 2'B } & 119.9 & 97.2 & 111.2\end{array}$

3'A $120.9 \quad 102.1 \quad 114.9$

$\begin{array}{llll}\text { 3'B } & 116.8 & 99.4 & 114.9\end{array}$

$\begin{array}{llll}\text { 4'A } & 122.3 & 101.9 & 112.7\end{array}$

$\begin{array}{llll}\text { 4'B } & 116.9 & 98.9 & 114.4\end{array}$

$\begin{array}{llll}\text { 5'A } & 119.1 & 101.8 & 115.9\end{array}$

$\begin{array}{llll}\text { 5'B } & 115.2 & 99.6 & 114.2\end{array}$ 


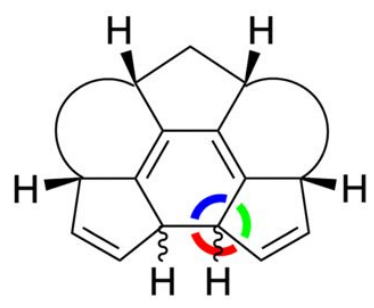

$\phi_{1}$

$\phi_{2}$

$\phi_{3}$

$\begin{array}{llll}1 \mathrm{~A} & 119.8 & 99.5 & 115.0\end{array}$

$\begin{array}{llrr}\text { 1B } & 139.4 & 100.2 & 112.8\end{array}$

$\begin{array}{llll}2 \mathrm{~A} & 119.5 & 100.4 & 114.7\end{array}$

$\begin{array}{llll}\text { 2B } & 138.6 & 99.2 & 112.5\end{array}$

$\begin{array}{llll}\text { 3A } & 120.8 & 101.9 & 115.5\end{array}$

$\begin{array}{llll}\text { 3B } & 127.8 & 100.3 & 113.9\end{array}$

$\begin{array}{llll}\text { 4A } & 120.1 & 101.6 & 115.3\end{array}$

$\begin{array}{llll}\text { 4B } & 128.5 & 100.0 & 113.5\end{array}$

$\begin{array}{llll}\mathbf{5 A} & 122.3 & 101.3 & 116.0\end{array}$

$\begin{array}{llll}5 B & 126.9 & 101.0 & 114.0\end{array}$

Figure S4. Bond angles (in ${ }^{\circ}$ ) around $\mathrm{C}^{3}$.

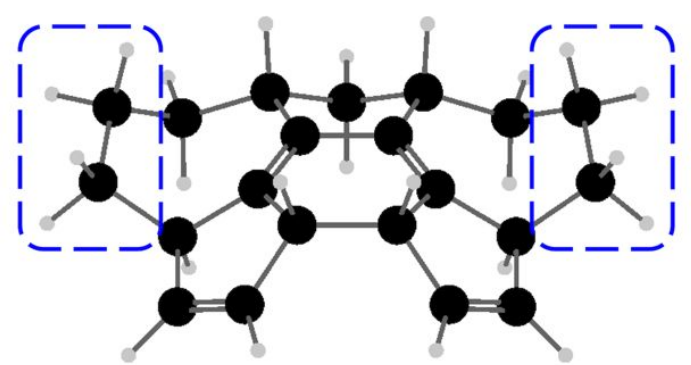

5'A

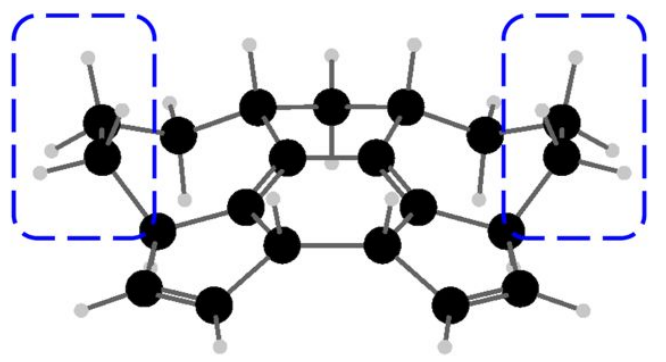

5'A

$2 \mathrm{nN}$

Figure S5. Dihedral angle inversion in 5'A owing to the application of an external force of 2 $\mathrm{nN}$. 


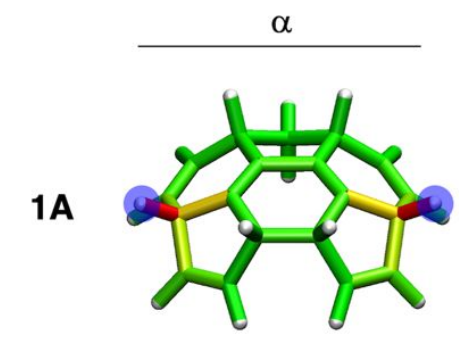

Bonds

Angles

$24.0 \%$

$25.3 \%$

Dihedrals

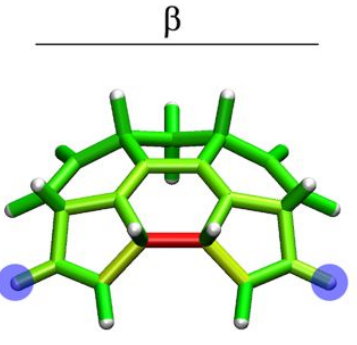

$16.6 \%$

$22.9 \%$

$60.5 \%$

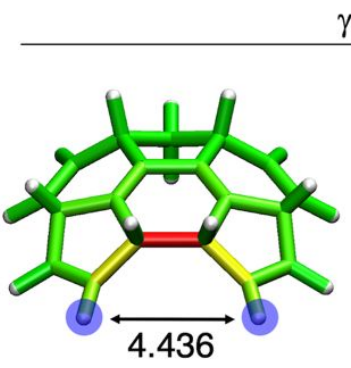

$18.9 \%$

$34.3 \%$

$46.8 \%$

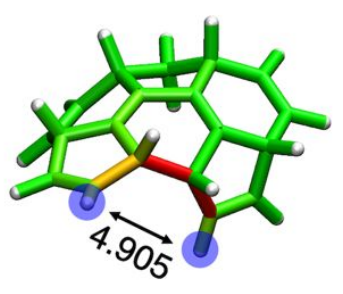

$10.6 \%$

$31.4 \%$

$58.0 \%$
2A

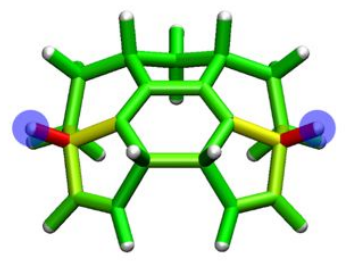

Bonds

Angles

Dihedrals
$30.3 \%$

$19.8 \%$

$49.9 \%$

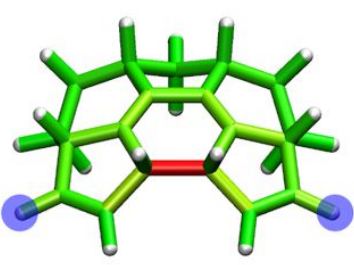

$17.9 \%$

$22.9 \%$

$59.2 \%$

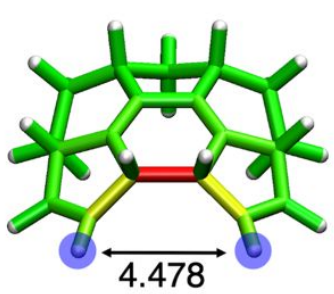

$21.4 \%$

$32.3 \%$

$46.2 \%$

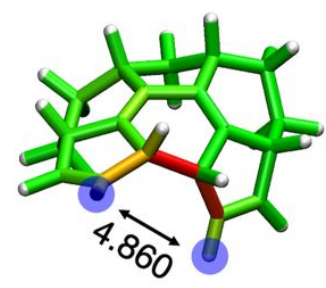

$13.7 \%$

$30.7 \%$

$55.6 \%$

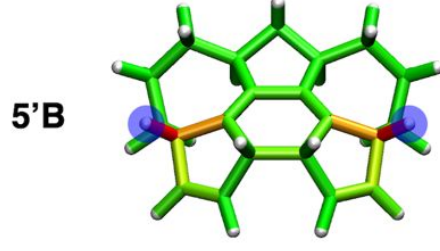

Bonds

Angles Dihedrals

$23.5 \%$
$22.9 \%$
$53.6 \%$

$23.5 \%$

$53.6 \%$

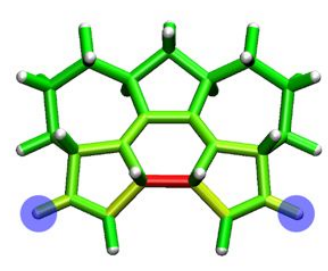

$23.5 \%$

$26.6 \%$

$49.9 \%$

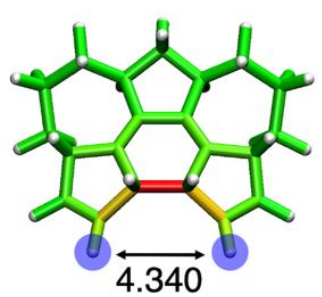

$20.4 \%$

$39.7 \%$

$39.9 \%$

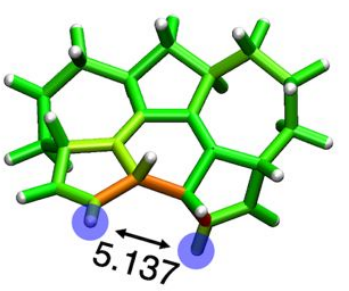

$10.4 \%$

$26.9 \%$

$62.7 \%$

\section{Stress energy}

Figure S6. Distribution of stress energy in $\mathbf{1 A}, \mathbf{2 A}$ and $\mathbf{5}^{\prime} \mathbf{B}\left(F_{\text {ext }}=2 \mathrm{nN}\right)$, and the fraction of stress energy in bonds, angles and dihedral angles. The pulling positions are indicated in blue and distances are in $\AA$. 
Table S1. Cartesian coordinates of each structure optimized at the wB97X-D3/Def2-TZ2P level of theory. The applied force, point group symmetry, electronic energy ( $E$ in a.u.), total enthalpy $\left(H\right.$ in a.u.), Gibbs energy ( $G$ in a.u.), number of imaginary frequencies $\left(n_{\text {imag }}\right)$ and imaginary vibrational frequency (for transitions states) are indicated.

$$
\begin{aligned}
& 1\left(C_{1}, 0 \mathrm{nN}\right) \\
& E=-733.58963142 \\
& H=-733.28201867 \\
& G=-733.33563324 \\
& \mathrm{n}_{\text {imag }}=0
\end{aligned}
$$

C -3.17076 -0.85437 2.85882

H -3.47064 -1.75413 3.42087

C - $2.20802-1.264691 .78915$

H - $1.23584-1.695781 .98599$

C - $-2.76731-1.059080 .59062$

H - $2.31722-1.27820-0.36877$

C $-4.36921-0.350632 .07757$

C -5.409420.334132.80953

C -6.65416 0.753792 .50162

C -7.51780 0.59455 1.35467

C -8.88984 1.23444 1.43967

H -9.490350.57500 2.09273

C -9.44309 1.063730 .06198

C - $8.586810 .32919-0.66063$

H -10.41695 $1.41692-0.24832$

H -8.73851 -0.00778 -1.67775

C -5.06532 0.747014.24057

H -5.39480 -0.06962 4.90444

C -7.22370 1.591393 .64903

H -7.87935 0.94327 4.25434 


$$
\begin{aligned}
& \text { C -8.04684 2.74565 } 3.14704 \\
& \text { H -7.98022 } 3.697603 .66397 \\
& \text { C -8.83678 2.58784 } 2.09322 \\
& \text { H -9.44444 } 3.395981 .70338 \\
& \text { C -2.70630 0.19142 } 3.84313 \\
& \text { H -1.64270 0.30416 4.01907 } \\
& \text { C -3.59694 0.94514 } 4.47301 \\
& \text { H -3.28152 1.69564 5.19099 } \\
& \text { C -5.98263 1.94672 4.46788 } \\
& \text { H -6.19834 2.13424 } 5.52163 \\
& \text { H -5.51521 2.842984.04732 } \\
& \text { C -7.39341 0.04267 0.12787 } \\
& \text { H -6.55506 -0.52507 -0.24119 } \\
& \text { C -4.09550 -0.47999 } 0.76207 \\
& \text { H -4.70578 -0.13129-0.05593 } \\
& 1\left(C_{\mathrm{s}}, 2 \mathrm{nN}\right) \\
& E=-733.57664062 \\
& H=-733.27106801 \\
& G=-733.32450798 \\
& \mathrm{n}_{\text {imag }}=0
\end{aligned}
$$

C 0.428130 .000183 .23608

H $1.35524-0.592423 .32198$

C 0.557651 .190464 .15631

H 0.588641 .143035 .27334

C 0.657612 .282963 .38004

H 0.786633 .299563 .72992

C 0.401090 .593921 .81328

C $0.10450-0.313300 .68613$

C $0.10448-0.31329-0.68612$

C $0.400980 .59396-1.81327$ 


$$
\begin{aligned}
& \text { C } 0.428200 .00022-3.23605 \\
& \text { H } 1.35541-0.59225-3.32189 \\
& \text { C } 0.557601 .19050-4.15629 \\
& \text { C } 0.657242 .28304-3.38003 \\
& \text { H } 0.588691 .14306-5.27332 \\
& \text { H } 0.786063 .29966-3.72992 \\
& \text { C -0.30609-1.71775 1.18423 } \\
& \text { H } 0.63162-2.271921 .36044 \\
& \text { C - } 0.30601-1.71777-1.18421 \\
& \text { H } 0.63174-2.27191-1.36032 \\
& \text { C - } 1.08437-1.72610-2.46612 \\
& \text { H -1.91728 -2.41554 -2.55989 } \\
& \text { C }-0.73810-0.91975-3.45600 \\
& \text { H -1.26653 -0.90016 -4.40174 } \\
& \text { C -0.73831 -0.91963 } 3.45595 \\
& \text { H -1.26682 -0.89994 } 4.40165 \\
& \text { C - } 1.08456-1.725982 .46607 \\
& \text { H -1.91755 -2.41533 } 2.55979 \\
& \text { C - } 1.02100-2.33405-0.00001 \\
& \text { H -0.99947 -3.42574 } 0.00001 \\
& \text { H -2.06746 -2.01114-0.00005 } \\
& \text { C } 0.554891 .92706-1.95771 \\
& \text { H } 0.560652 .65636-1.15857 \\
& \text { C } 0.555281 .926981 .95772 \\
& \text { H } 0.561272 .656271 .15856 \\
& 1^{\prime}\left(C_{\mathrm{s}}, 0 \mathrm{nN}\right) \\
& E=-733.58975465 \\
& H=-733.28192665 \\
& G=-733.33584887 \\
& \mathrm{n}_{\text {imag }}=0
\end{aligned}
$$




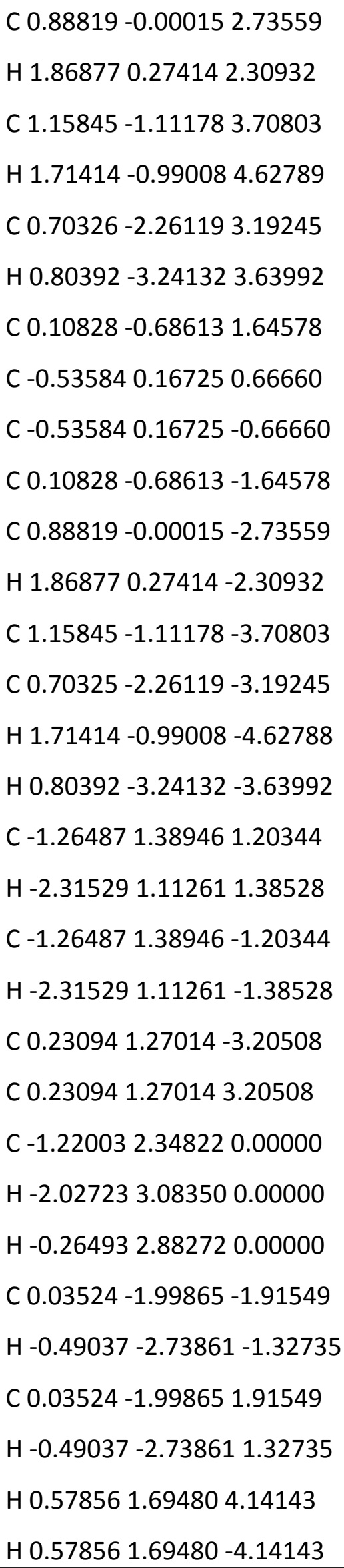




$$
\begin{aligned}
& \text { C -0.70619 } 1.89607-2.50155 \\
& \text { H -1.12554 2.82166 -2.88624 } \\
& \text { C -0.70619 } 1.896072 .50155 \\
& \text { H -1.12555 2.82166 2.88624 } \\
& 1^{\prime}\left(C_{s}, 1.8 \mathrm{nN}\right) \\
& E=-733.57165261 \\
& H=-733.26550994 \\
& G=-733.31928554 \\
& \mathrm{n}_{\text {imag }}=0
\end{aligned}
$$

C $0.81202-0.002713 .10942$

H 1.895090 .175722 .99823

C $0.66758-1.076294 .17806$

H $0.84487-0.951465 .27084$

C $0.31127-2.211313 .55692$

H 0.16423 -3.17330 4.03136

C $0.33015-0.680851 .82454$

C -0.13129 0.142950.67899

C -0.13153 0.14257 -0.67884

C $0.32831-0.68229-1.82421$

C $0.81330-0.00499-3.10834$

H $1.896840 .16991-2.99620$

C $0.66613-1.07739-4.17778$

C $0.30400-2.21125-3.55788$

H $0.84480-0.95247-5.27033$

H $0.15289-3.17215$-4.03325

C - -1.012821 .303741 .17526$

H -2.03248 0.89575 1.27773

C -1.01112 $1.30446-1.17586$

H -2.03094 0.89745 -1.28065

C $0.161601 .31621-3.37658$ 


$$
\begin{aligned}
& \text { C } 0.156171 .316313 .37798 \\
& \text { C -1.02166 2.26908 -0.00001 } \\
& \text { H -1.87935 } 2.94529-0.00048 \\
& \text { H -0.10362 2.865500.00090 } \\
& \text { C } 0.08941-1.97213-2.12408 \\
& \text { H -0.30170 -2.71298 -1.43850 } \\
& \text { C } 0.09659-1.971952 .12317 \\
& \text { H -0.29037 -2.71405 } 1.43659 \\
& \text { H } 0.375811 .800244 .32445 \\
& \text { H } 0.383821 .80029-4.32237 \\
& \text { C -0.63910 } 1.89879-2.49798 \\
& \text { H -1.08747 } 2.85963-2.73602 \\
& \text { C -0.644301.89777 } 2.49846 \\
& \text { H -1.095142.85746 2.73647 } \\
& 1^{\prime}\left(C_{s}, 2.0 \mathrm{nN}\right) \\
& E=-733.56914323 \\
& H=-733.26307761 \\
& G=-733.3165495 \\
& \mathrm{n}_{\text {imag }}=0
\end{aligned}
$$

C 0.813040 .000213 .12742

H 1.897740 .174143 .02623

C $0.65269-1.066124 .20474$

H $0.81764-0.938095 .30296$

C $0.29667-2.202133 .58465$

H 0.13852 -3.16089 4.06227

C $0.34044-0.683841 .83889$

C -0.112940.133750.68030

C -0.11294 0.13375 -0.68030

C $0.34044-0.68384-1.83889$

C $0.813040 .00021-3.12742$ 


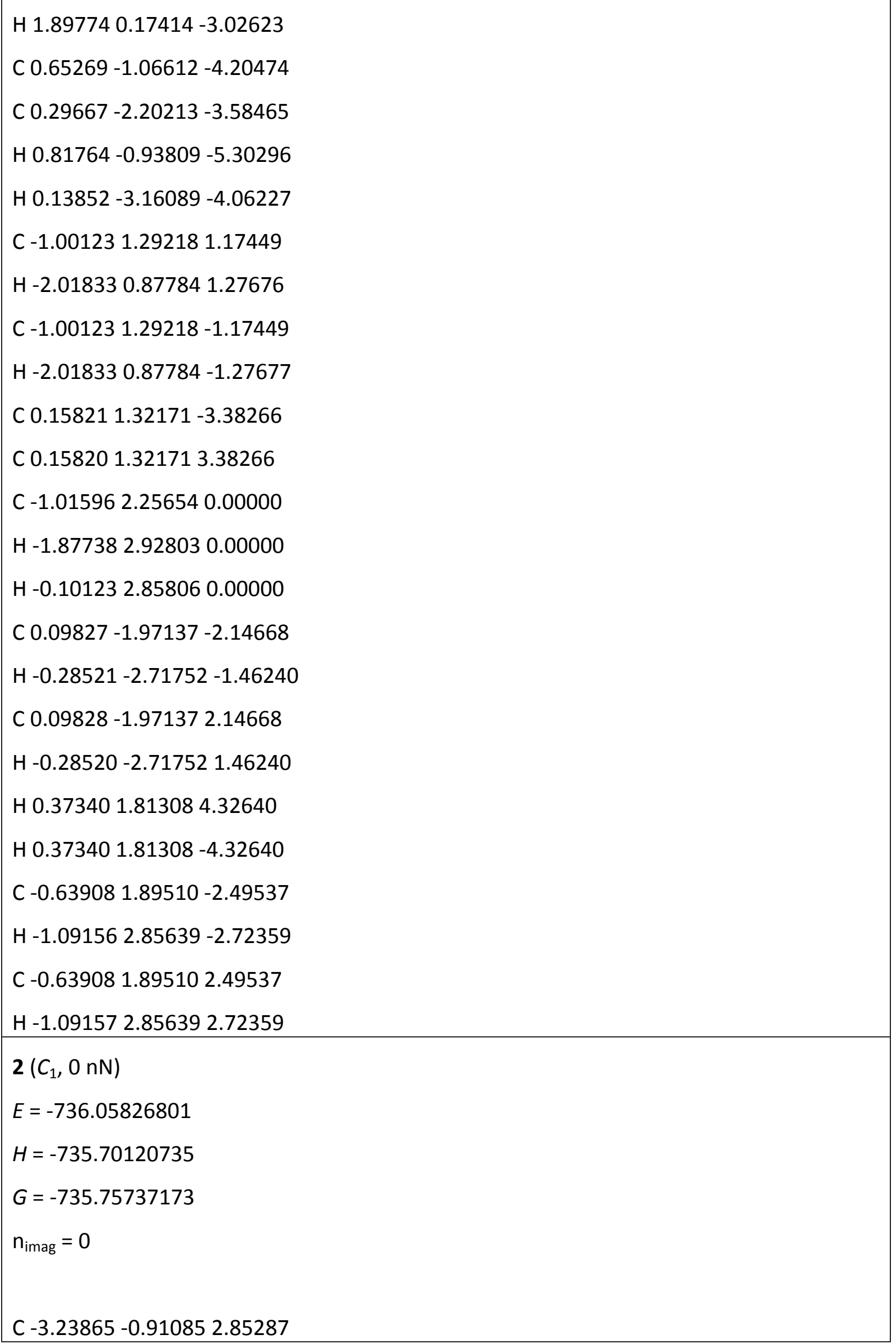




$$
\begin{aligned}
& \text { H -3.70393 -1.765373.36357 } \\
& \text { C -2.29227 -1.42456 } 1.81373 \\
& \text { H -1.39695 -1.98839 2.03875 } \\
& \text { C - } 2.73556-1.077720 .59841 \\
& \text { H -2.25777 -1.30886 -0.34472 } \\
& \text { C - } 4.31006-0.228452 .03320 \\
& \text { C -5.35760 0.44969 } 2.76220 \\
& \text { C -6.60453 } 0.859442 .45635 \\
& \text { C -7.46270 0.71051 1.30121 } \\
& \text { C -8.90574 } 1.113421 .49275 \\
& \text { H -9.35467 0.40055 2.20052 } \\
& \text { C -9.51189 } 0.878930 .14678 \\
& \text { C -8.57692 } 0.38571-0.67660 \\
& \text { H -10.55007 } 1.06710-0.09156 \\
& \text { H -8.72370 } 0.10203-1.71072 \\
& \text { C -5.03290 0.75971 4.21555 } \\
& \text { H -5.23008 -0.14151 4.81617 } \\
& \text { C -7.24311 } 1.566803 .64189 \\
& \text { H -7.940740.87275 4.13316 } \\
& \text { C -8.01765 } 2.809203 .19964 \\
& \text { H -7.28663 } 3.538492 .83865 \\
& \text { C -9.04651 2.52748 2.07907 } \\
& \text { H -8.91170 } 3.249871 .27138 \\
& \text { C -2.616230.01839 } 3.92550 \\
& \text { H -1.70336 } 0.462523 .52468 \\
& \text { C -3.56743 1.14136 4.37917 } \\
& \text { H -3.388542.03600 } 3.77398 \\
& \text { C -6.05516 1.841374.56881 } \\
& \text { H -6.32547 } 1.850675 .62635 \\
& \text { H -5.63156 2.82274 } 4.32692 \\
& \text { C - } 7.302780 .284480 .02966
\end{aligned}
$$




$$
\begin{aligned}
& \mathrm{H}-6.39548-0.08373-0.42106 \\
& \mathrm{C}-3.98240-0.327310 .72823 \\
& \mathrm{H}-4.507430 .11850-0.10239 \\
& \mathrm{H}-3.354231 .415805 .41553 \\
& \mathrm{H}-2.31445-0.589954 .78104 \\
& \mathrm{H}-8.508193 .265414 .06305 \\
& \mathrm{H}-10.066812 .659522 .44561 \\
& \mathbf{2}\left(C_{\mathrm{s}}, 2 \mathrm{nN}\right) \\
& E=-736.04437365 \\
& H=-735.68904916 \\
& G=-735.74505423 \\
& \mathrm{n}_{\text {imag }}=0
\end{aligned}
$$

C $0.42187-0.000803 .22923$

H $1.26861-0.698583 .29663$

C 0.683501 .167914 .14887

H 0.719911 .116185 .26584

C 0.832452 .258713 .37684

H 1.031883 .263413 .72843

C 0.422840 .598461 .81588

C $0.11792-0.309820 .68590$

C $0.11792-0.30982-0.68590$

C $0.422840 .59846-1.81588$

C $0.42187-0.00080-3.22923$

H $1.26860-0.69858-3.29663$

C $0.683501 .16791-4.14887$

C $0.832462 .25871-3.37684$

H 0.719911 .11618 -5.26584

H $1.031893 .26341-3.72843$

C -0.29147 -1.69092 1.21191

H 0.61044 -2.18397 1.59995 


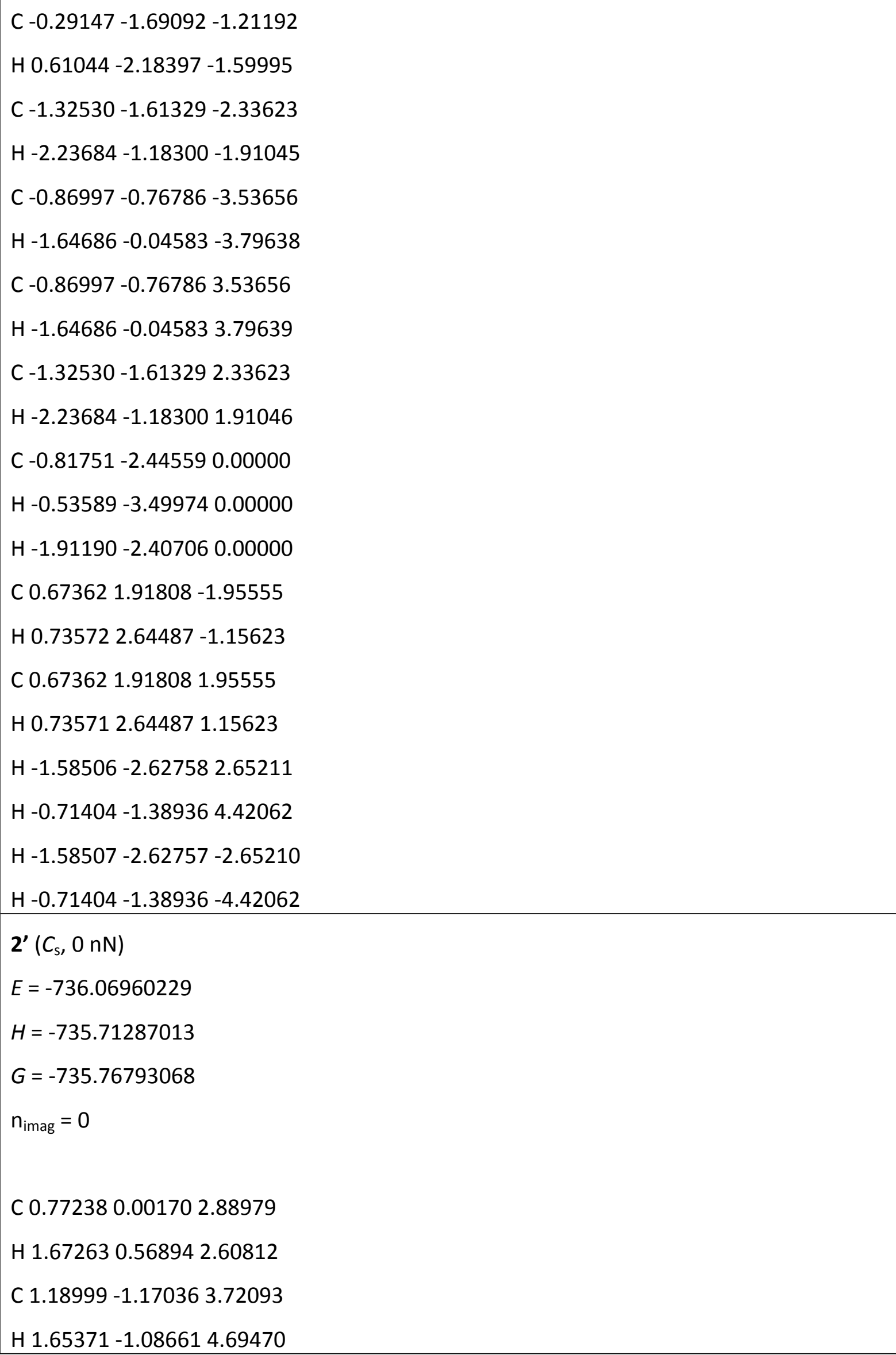




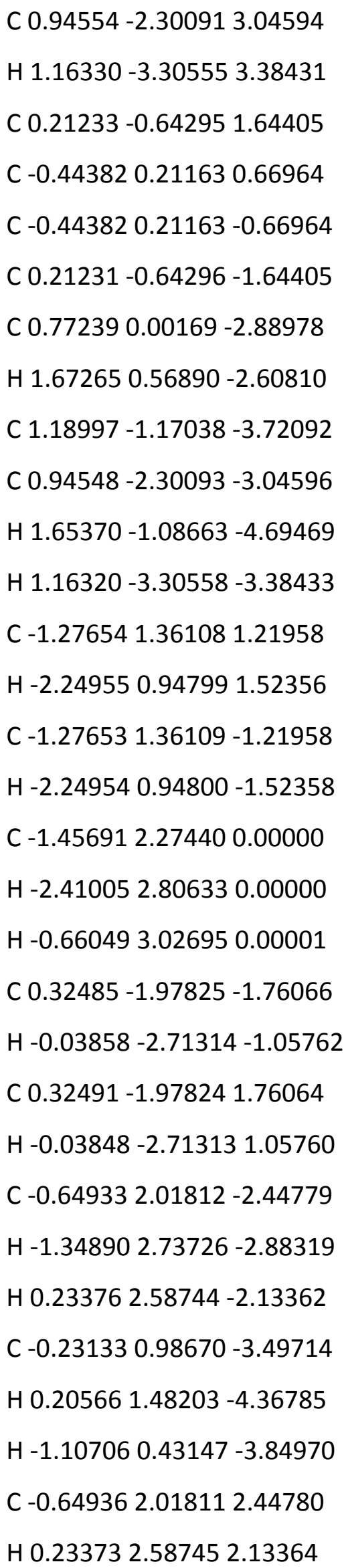




$$
\begin{aligned}
& H-1.348942 .737242 .88320 \\
& C-0.231370 .986703 .49714 \\
& H-1.107080 .431443 .84969 \\
& H 0.205611 .482024 .36787 \\
& \mathbf{2}^{\prime}\left(C_{s}, 2 \mathrm{nN}\right) \\
& E=-736.05083639 \\
& H=-735.69590673 \\
& G=-735.75048199 \\
& \mathrm{n}_{\text {imag }}=0
\end{aligned}
$$

C $0.62227-0.000213 .20243$

H 1.587390 .527773 .15296

C $0.77440-1.175094 .14188$

H 0.88681 -1.10620 5.25278

C $0.72000-2.293133 .39928$

H $0.80805-3.307313 .76848$

C $0.38049-0.631391 .81860$

C - 0.114180 .199720 .68441

C -0.11418 0.19972 -0.68441

C $0.38049-0.63139-1.81860$

C $0.62227-0.00021-3.20243$

H $1.587390 .52777-3.15296$

C $0.77440-1.17509-4.14188$

C $0.72000-2.29313-3.39928$

H 0.88681 -1.10620 -5.25278

H 0.80805 -3.30731 -3.76848

C -1.03907 1.32557 1.18764

H -2.01426 0.85148 1.38333

C -1.03907 1.32557 -1.18764

H - $2.014260 .85148-1.38333$

C -1.17419 2.25449 0.00000 


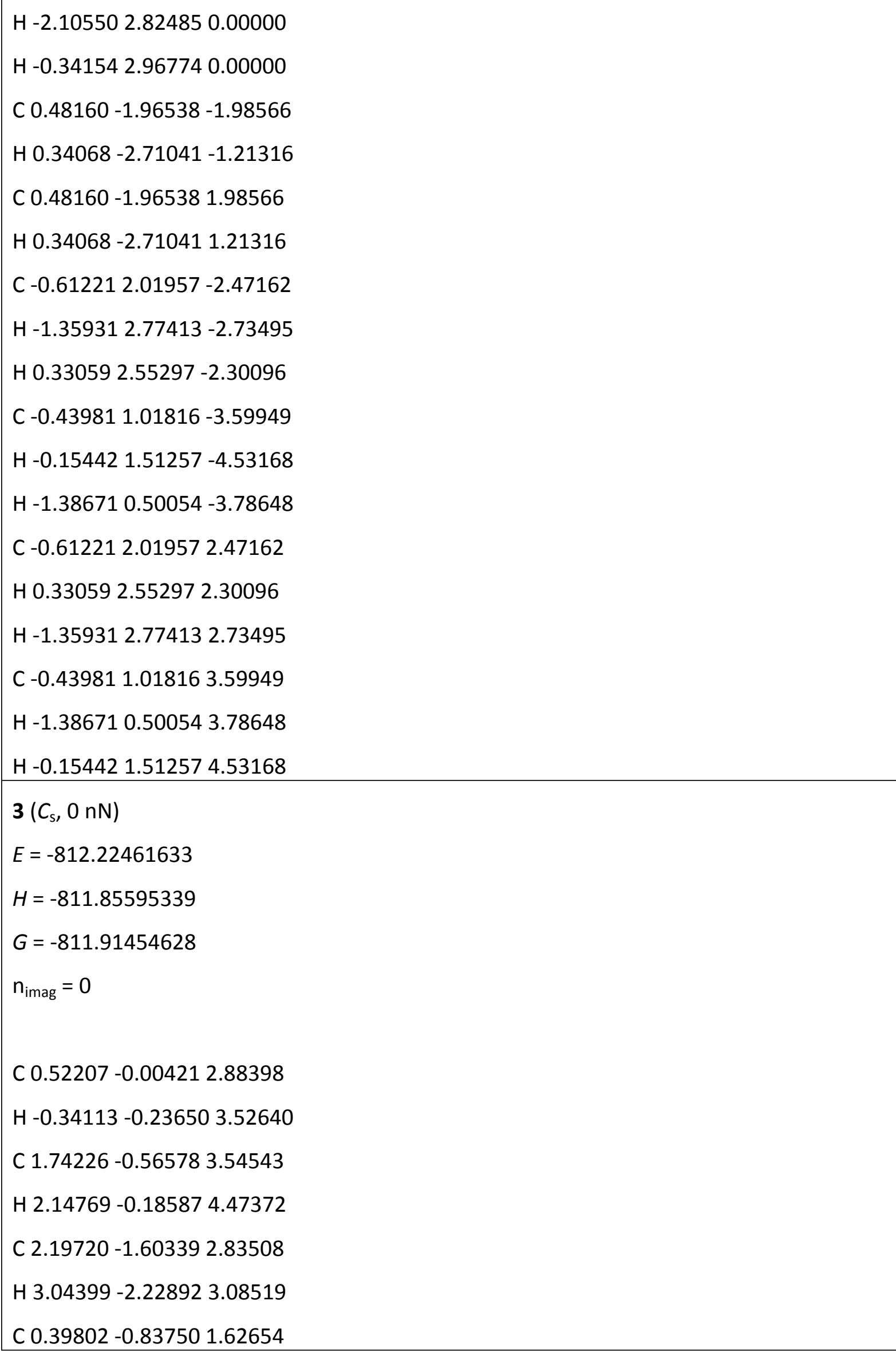




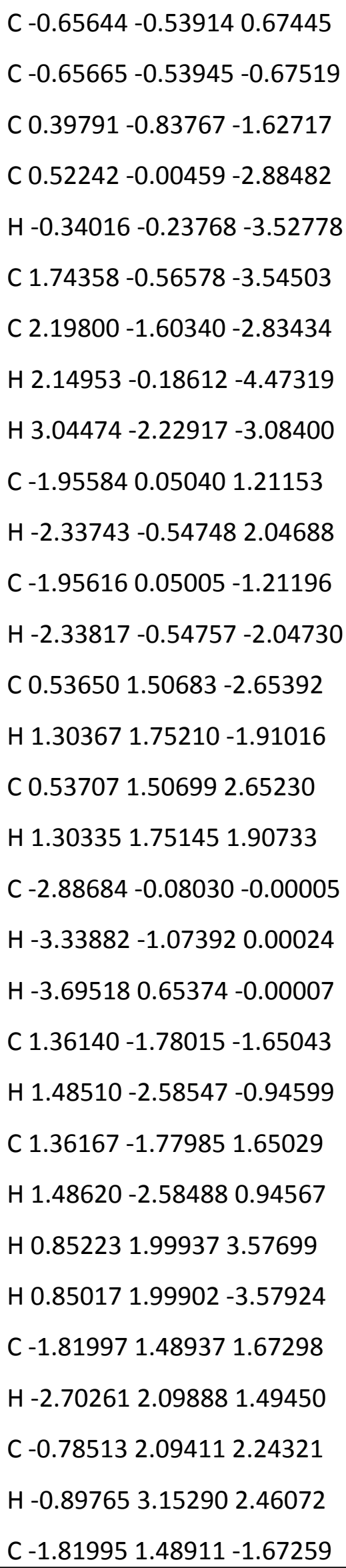




$$
\begin{aligned}
& \text { H -2.70104 2.09965 -1.49011 } \\
& \text { H -0.89668 3.15350 -2.45703 } \\
& 3\left(C_{s}, 2 \mathrm{nN}\right) \\
& E=-812.20497412 \\
& H=-811.83777697 \\
& G=-811.89563301 \\
& \mathrm{n}_{\text {imag }}=0
\end{aligned}
$$$$
\text { C - } 0.785272 .09387-2.24304
$$

C 0.318110 .000483 .24979

H -0.02865 -1.00169 3.52430

C 1.497000 .355334 .12720

H 1.460710 .420305 .24356

C 2.554530 .555583 .32473

H 3.554410 .824163 .64193

C $0.88531-0.018291 .81677$

C $0.04712-0.497510 .68650$

C $0.04712-0.49751-0.68650$

C $0.88531-0.01829-1.81677$

C $0.318110 .00048-3.24979$

H -0.02864 -1.00169 -3.52430

C $1.497000 .35533-4.12720$

С $2.554530 .55558-3.32473$

H $1.460710 .42031-5.24356$

H $3.554410 .82416-3.64193$

C -1.21978 -1.20654 1.19948

H -0.95829 -1.89560 2.00474

C -1.21978 -1.20654-1.19948

H -0.95829 -1.89560 -2.00473

C -0.84057 0.98839-3.44159

H -0.48489 1.99531 -3.19207 


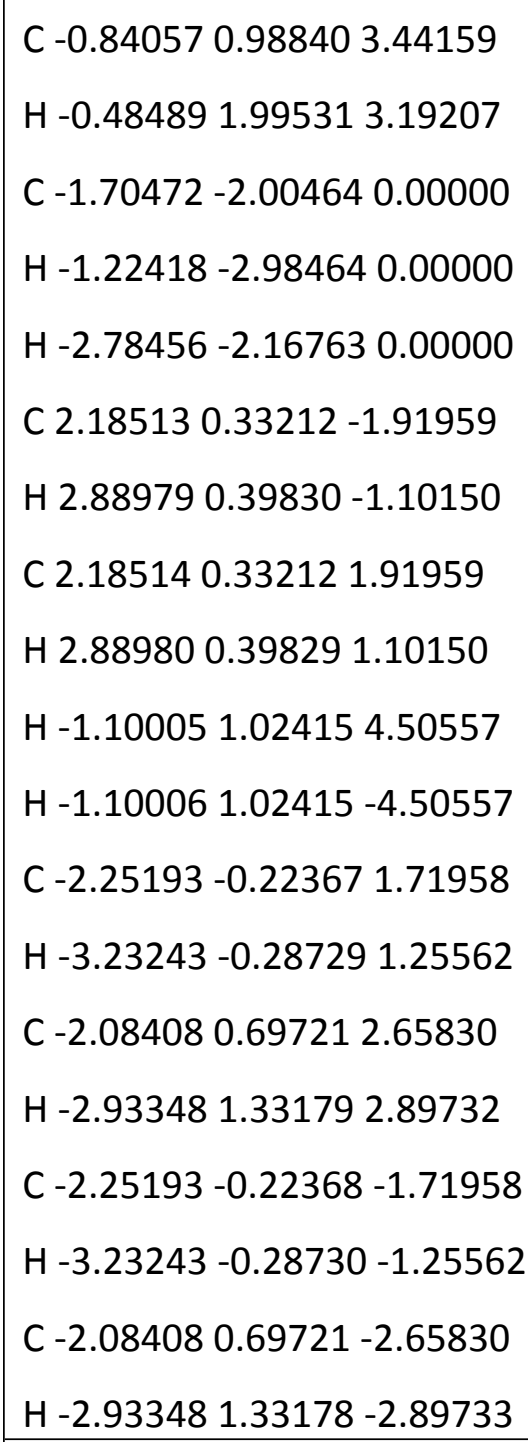

3' $\left(C_{\mathrm{s}}, 0 \mathrm{nN}\right)$

$E=-812.22411532$

$H=-811.85554142$

$G=-811.91398696$

$\mathrm{n}_{\text {imag }}=0$

C 1.058030 .000672 .63562

H 1.774050 .655482 .11967

C $1.81276-1.072993 .36172$

H $2.48132-0.875744 .18921$

C $1.53892-2.263202 .81880$

H 1.94628 -3.21688 3.12753 


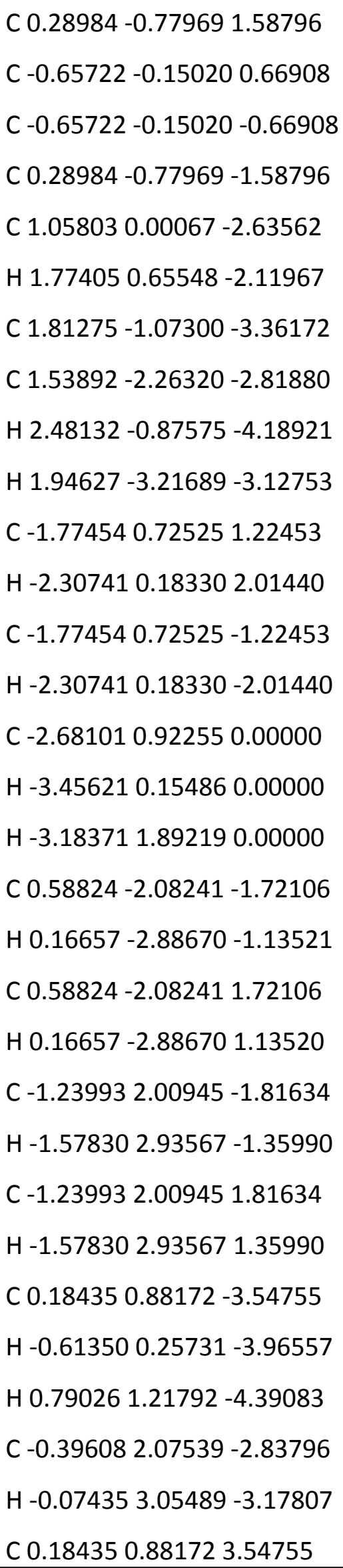




$$
\begin{aligned}
& \text { H -0.61350 0.25731 } 3.96557 \\
& \text { H } 0.790261 .217924 .39083 \\
& \text { C -0.39609 2.075392.83796 } \\
& \text { H -0.07435 } 3.054893 .17807 \\
& \text { 3' }\left(C_{s}, 2 \mathrm{nN}\right) \\
& E=-812.1930547 \\
& H=-811.82633296 \\
& G=-811.88405146 \\
& \mathrm{n}_{\text {imag }}=0
\end{aligned}
$$

C 0.827030 .000243 .19529

H 1.727910 .612313 .03330

C $1.21181-1.129204 .13253$

H 1.36024 -1.02309 5.23622

C $1.28659-2.256613 .41129$

H 1.55589 -3.23644 3.78477

C $0.56221-0.707371 .83636$

C - $0.19426-0.117470 .68221$

C -0.19426 -0.11747 -0.68221

C $0.56220-0.70737-1.83636$

C $0.827030 .00024-3.19529$

H $1.727920 .61231-3.03330$

C $1.21181-1.12920-4.13253$

C $1.28659-2.25661-3.41129$

H 1.36024 -1.02309 -5.23622

H 1.55588 -3.23645 -3.78477

C -1.432630.64637 1.19827

H -1.885190.06257 2.00536

C -1.43263 0.64637-1.19827

H -1.88519 0.06257-2.00536

C - 2.366210 .699450 .00000 


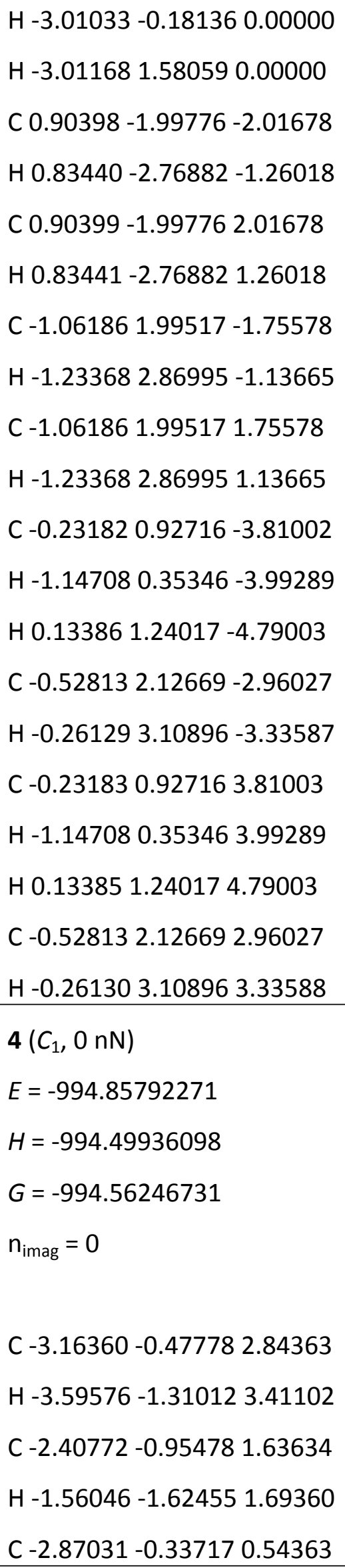




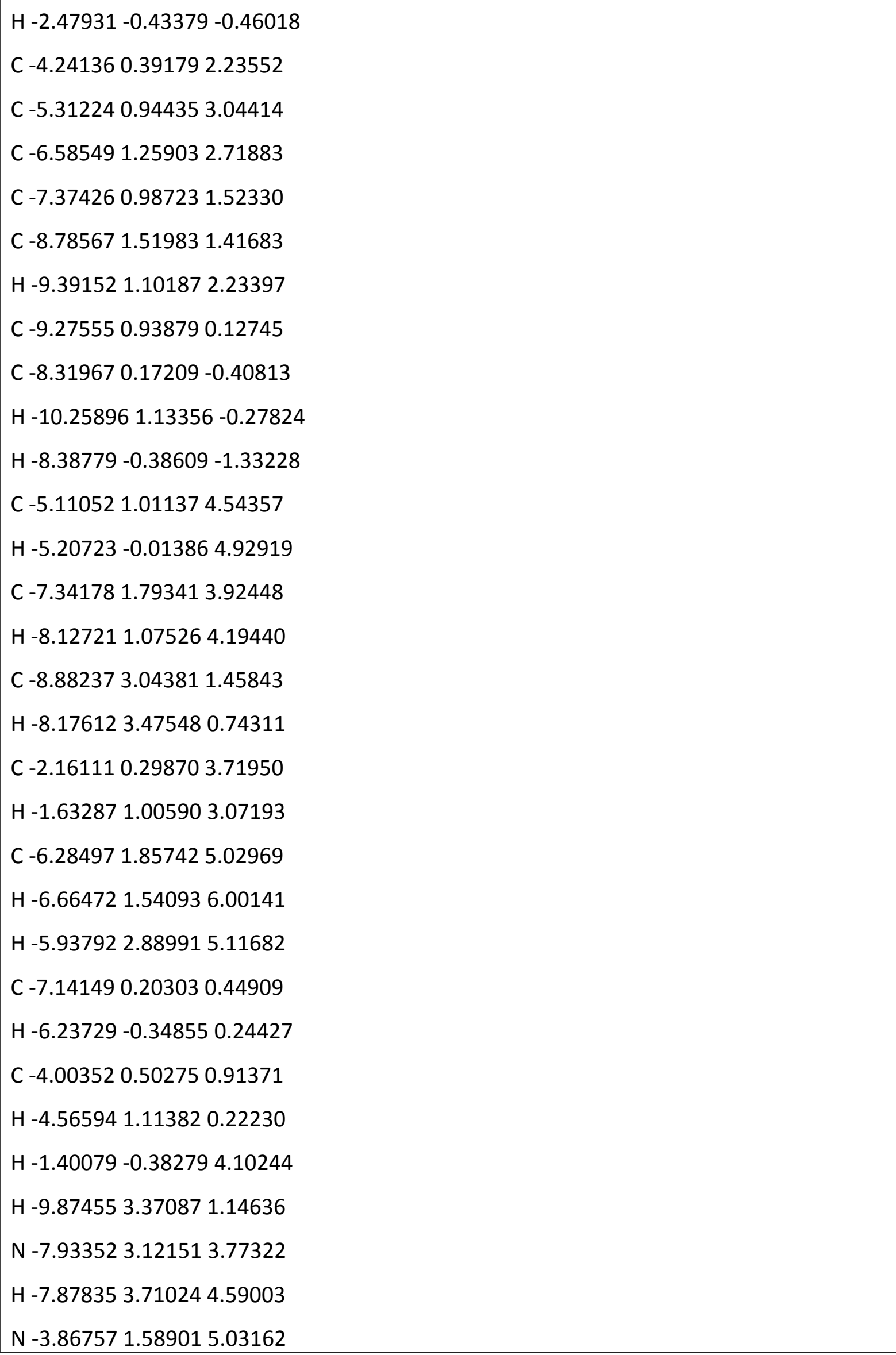




$$
\begin{aligned}
& \text { H -3.95993 } 2.167255 .85334 \\
& \text { C -8.64457 } 3.734102 .78546 \\
& \text { C - } 2.585081 .142414 .90597 \\
& \text { O -9.070824.85925 2.95994 } \\
& \text { O -1.73269 } 1.514035 .68757 \\
& 4\left(C_{s}, 2 \mathrm{nN}\right) \\
& E=-994.84432714 \\
& H=-994.48758114 \\
& G=-994.5500613 \\
& \mathrm{n}_{\text {imag }}=0 \\
& \text { C - }-0.69380-0.093883 .25201 \\
& \text { H -0.822810.96298 3.51114 } \\
& \text { C -1.61448 -0.90902 } 4.13202 \\
& \text { H -1.56032 -0.93921 } 5.24884 \\
& \text { C - } 2.47427-1.558933 .33128 \\
& \text { H -3.26203 -2.22856 } 3.65159 \\
& \text { C -1.19449 -0.35227 } 1.81900 \\
& \text { C - } 0.596260 .394060 .68662 \\
& \text { C -0.59624 0.39405 -0.68661 } \\
& \text { C -1.19443 -0.35233 -1.81898 } \\
& \text { C }-0.69381-0.09388-3.25200 \\
& \text { H -0.82292 } 0.96298-3.51111 \\
& \text { C }-1.61446-0.90908-4.13199 \\
& \text { C - } 2.47411-1.55914-3.33123 \\
& \text { H -1.56034 -0.93924 -5.24881 } \\
& \text { H -3.26179 -2.22887 -3.65151 } \\
& \text { C } 0.304901 .526051 .20333 \\
& \text { H -0.205572.08230 } 1.99099 \\
& \text { C } 0.304881 .52607-1.20331 \\
& \text { H -0.20563 2.08234 -1.99095 }
\end{aligned}
$$


C $0.76676-0.50209-3.47680$

H 0.88003 -1.56226 -3.23256

C $0.76675-0.502203 .47673$

H 0.87994 -1.56237 3.23244

C 0.500392 .429300 .00002

H -0.27970 3.190070 .00003

H 1.457492 .955740 .00001

C -2.21889-1.22566-1.92368

H -2.80844 -1.61343 -1.10387

C -2.21907-1.22546 1.92373

H -2.80871 -1.61313 1.10394

H $1.02257-0.408004 .53343$

H $1.02253-0.40781-4.53350$

N $1.590441 .05489-1.70671$

H 2.422041 .44515 -1.29375

N 1.590461 .054801 .70669

H 2.422061 .445051 .29372

C $1.880450 .21064-2.73288$

C 1.880450 .210492 .73281

O $3.034700 .00008-3.04744$

O $3.03470-0.000143 .04733$

4' $\left(C_{\mathrm{s}}, 0 \mathrm{nN}\right)$

$E=-994.86839556$

$H=-994.51008516$

$G=-994.57283025$

$\mathrm{n}_{\text {imag }}=0$

C -0.35927-1.00477 2.72546

H 0.32047 -1.74969 2.28704

C -1.43835-1.73163 3.47152

H -1.24773 -2.33867 4.34610 


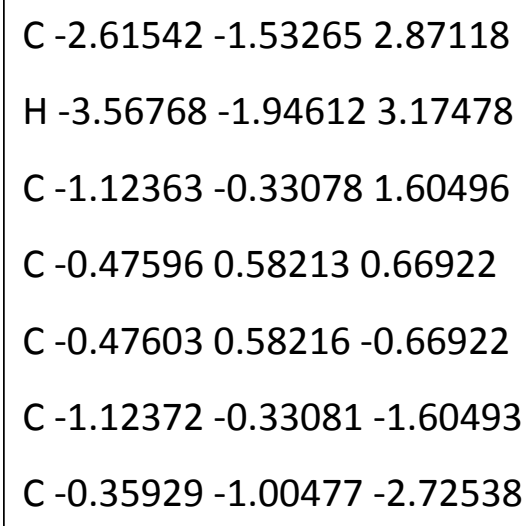
H 0.32047 -1.74965 -2.28695

C -1.43827-1.73168-3.47152

C -2.61539-1.53277-2.87125

H -1.24756 -2.33863 -4.34614

H -3.56761 -1.94622 -3.17498

C 0.422661 .676481 .22781

H -0.11145 2.25428 1.98828

C $0.422581 .67653-1.22785$

H -0.11158 2.25434-1.98828

C $0.709222 .54892-0.00002$

H 0.024113 .395560 .00003

H $1.719562 .96376-0.00005$

C - $2.42214-0.65580-1.71632$

H -3.21954 -0.29286-1.08444

C -2.42206 -0.65570 1.71627

H -3.21939 -0.29276 1.08429

H $2.527761 .36726-1.37076$

H 2.527791 .366961 .37058

C $0.47896-0.05820-3.60100$

H -0.12405 $0.81006-3.88345$

H $0.78332-0.56868-4.51277$

C $0.47905-0.058243 .60113$

H -0.123970.80994 3.88377

H $0.78352-0.568844 .51279$ 


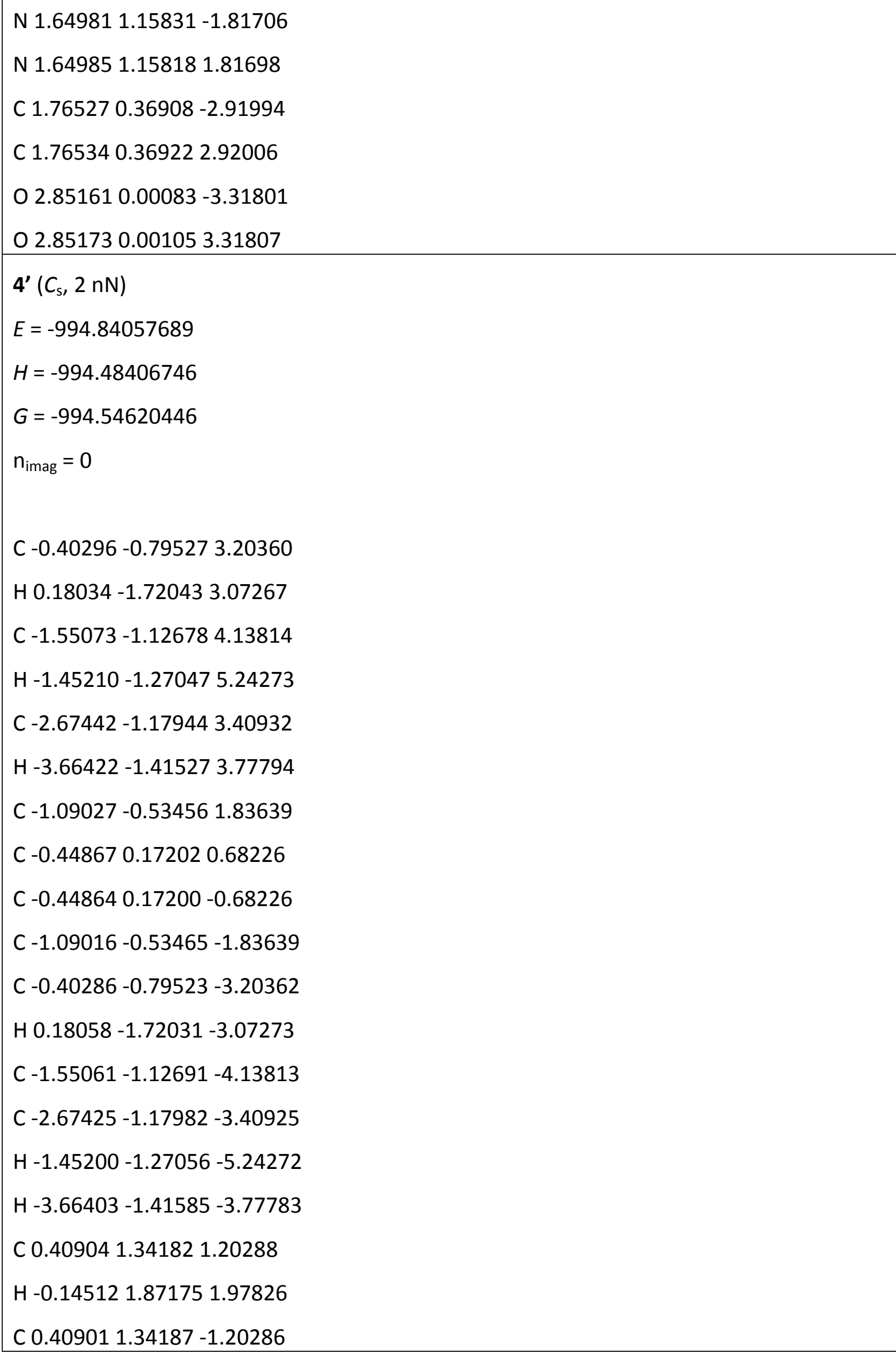




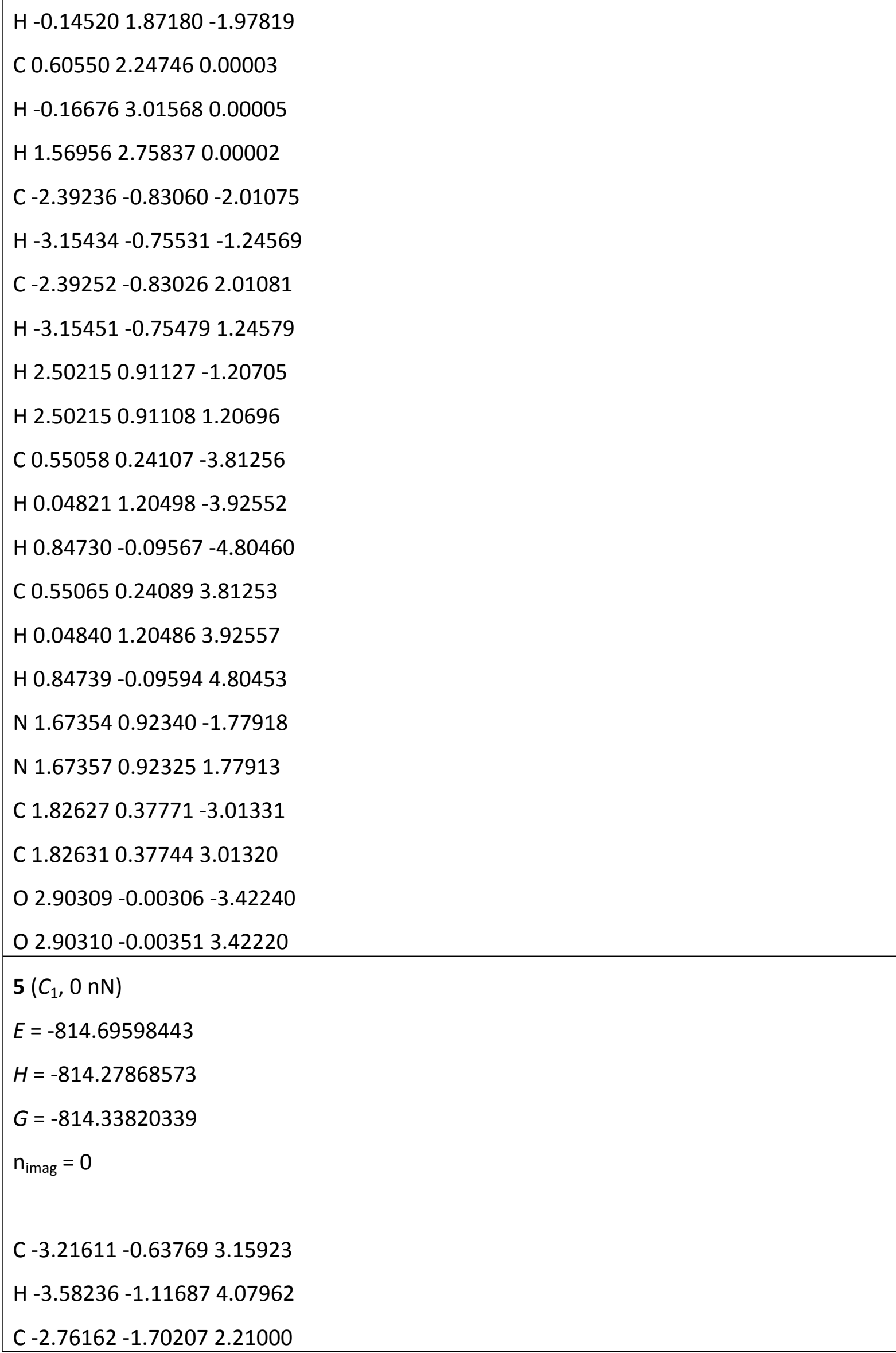




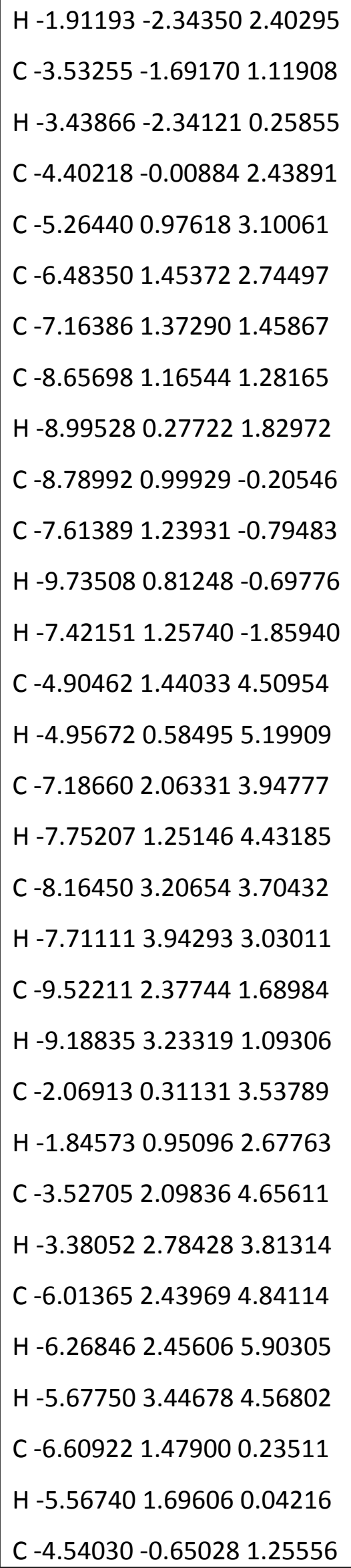




$$
\begin{aligned}
& H-5.29757-0.461910 .51282 \\
& H-3.557482 .723055 .55524 \\
& H-1.17398-0.290813 .71768 \\
& H-8.322423 .718524 .65902 \\
& H-10.551132 .168091 .38272 \\
& C-9.519552 .764703 .16170 \\
& H-10.238753 .579093 .28990 \\
& H-9.893901 .930913 .76762 \\
& C-2.337731 .157974 .77249 \\
& H-1.442821 .746134 .99696 \\
& H-2.489830 .494265 .63245 \\
& \hline 5\left(C_{s}, 2 n N\right) \\
& E=-814.67621248 \\
& H=-814.26039255 \\
& G=-814.31978417 \\
& n_{\text {imag }}=0
\end{aligned}
$$

C $0.42684-0.001843 .27528$

H 0.25032 -1.05533 3.52942

C 1.595420 .482334 .10035

H 1.591100 .531265 .21831

C 2.584430 .818213 .25783

H 3.559371 .199393 .53472

C 0.918510 .082071 .81078

C $0.08502-0.431280 .68991$

C $0.08479-0.43118-0.69011$

C $0.918240 .08207-1.81098$

C $0.42684-0.00187-3.27557$

H 0.25037 -1.05535 -3.52977

C $1.595680 .48235-4.10035$

C $2.584500 .81819-3.25758$ 


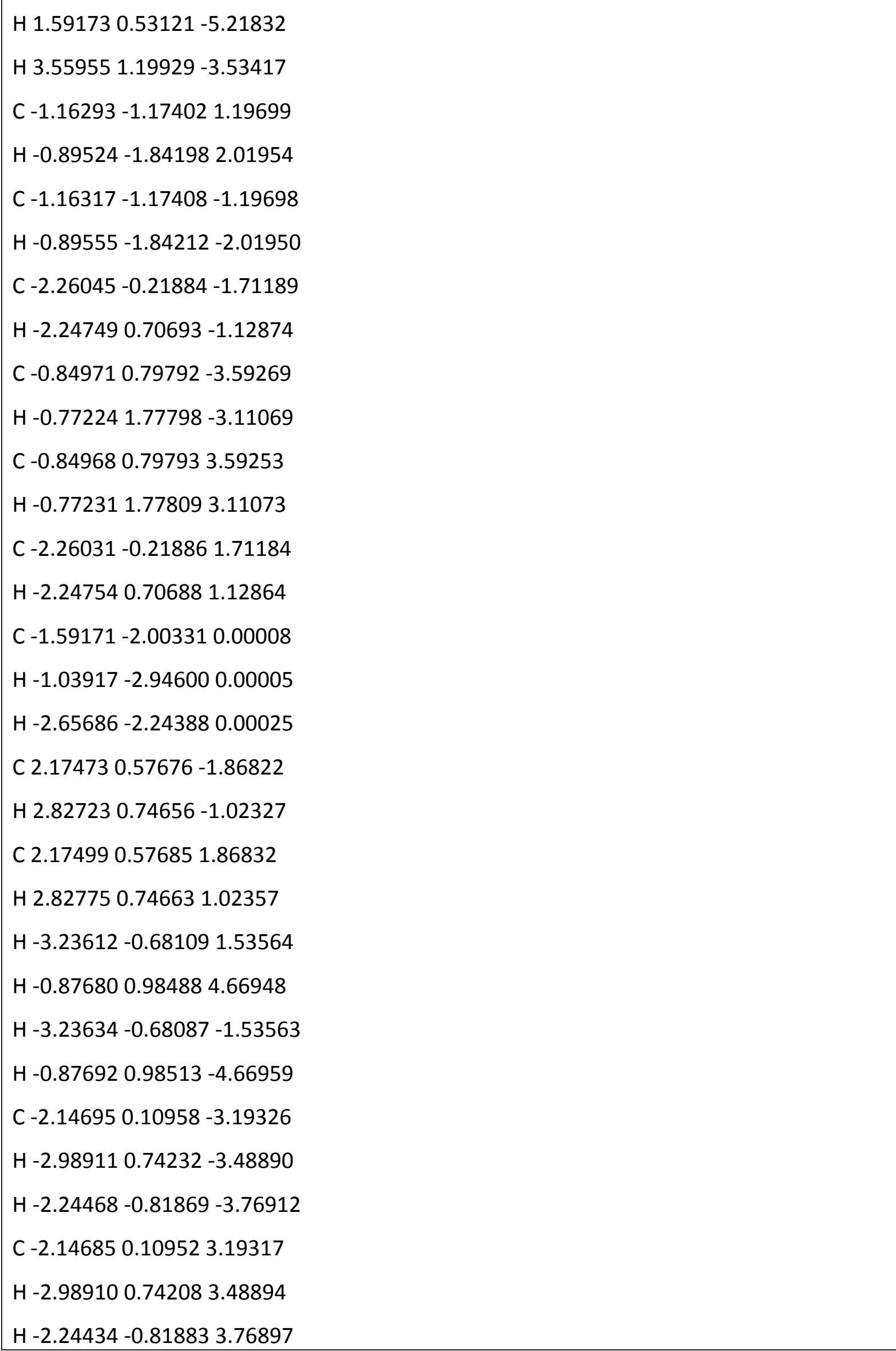




$$
\begin{aligned}
& 5^{\prime}\left(C_{1}, 0 \mathrm{nN}\right) \\
& E=-814.68580274 \\
& H=-814.2683675 \\
& G=-814.32820073 \\
& \mathrm{n}_{\text {imag }}=0
\end{aligned}
$$

C - 2.788860 .143752 .59417

H -2.302361.05229 2.20675

C -2.31657-0.96191 1.70446

H -1.29827 -1.32764 1.70200

C -3.31688-1.35053 0.90785

H -3.26622 -2.102930.13174

C - 4.269660 .261352 .25996

C -5.15827 1.21845 2.93564

C -6.44784 1.535392 .66753

C -7.41529 0.84569 1.81162

C -8.48923 1.525940 .97853

H -8.04399 2.171960.21120

C -9.204320.364380.35239

C -8.71272 -0.78389 0.82707

H -10.03100 0.48101 -0.33584

H -9.05979 -1.78007 0.58674

C -4.687342.01664 4.15841

H -4.89999 1.393205 .03986

C -6.91711 2.739423 .47183

H -7.65818 2.41878 4.21760

C -5.63710 3.20924 4.15966

H -5.81161 3.613325 .15906

H -5.185434.00795 3.55963

C -7.61539-0.48346 1.74148

H -7.05289 -1.22626 2.29084 


$$
\begin{array}{ccc}
C-4.51863 & -0.60359 & 1.24975 \\
\text { H }-5.46339 & -0.747230 .75299 \\
\text { C }-9.47808 & 2.341801 .83608 \\
\text { H }-9.71546 & 1.736642 .71606
\end{array}
$$$$
\text { H -10.41523 2.45181 } 1.28493
$$$$
\text { C -2.442770.01835 } 4.08093
$$$$
\text { H -3.22806 -0.55860 4.57645 }
$$$$
\text { H -1.52377 -0.56066 4.19557 }
$$$$
\text { C -7.52045 } 3.854732 .60797
$$$$
\text { H -6.92138 } 3.923351 .69305
$$$$
\text { H -7.37479 4.80716 } 3.12718
$$$$
\text { C -9.00099 } 3.731422 .25723
$$$$
\text { H -9.21874 } 4.445141 .45672
$$$$
\text { H -9.60295 } 4.049393 .11416
$$$$
\text { C -3.22756 2.45473 } 4.24682
$$

H -3.17068 3.31409 4.92209

H -2.90786 2.830393.26958

C - 2.266531 .381744 .75534

H -2.39028 1.26677 5.83639

H -1.24104 1.731824.60160

$5^{\prime}\left(\mathrm{C}_{1}, 2 \mathrm{nN}\right)$

$E=-814.66550984$

$H=-814.24976389$

$G=-814.30878695$

$\mathrm{n}_{\text {imag }}=0$

C -2.641110.08885 2.78355

H -2.13122 1.000042 .44114

C -1.97276-1.03316 2.03623

H -0.91112 -1.33612 2.21879

C - $2.85076-1.507361 .13846$ 


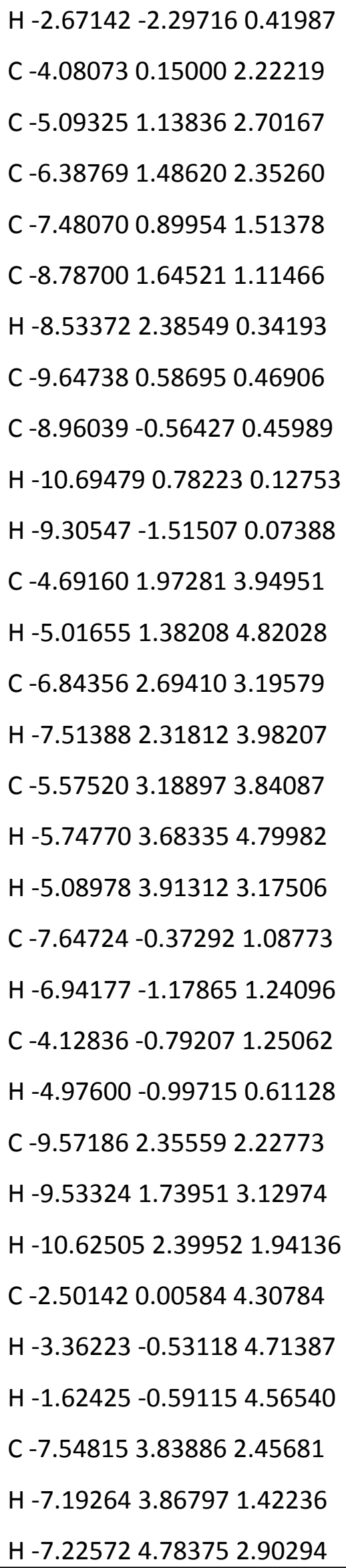


C -9.06665 3.76470 2.50379
H -9.48874 4.45907 1.77087
H -9.42411 4.09490 3.48391
C -3.25072 2.41730 4.20959
H -3.29813 3.32160 4.82369
H -2.78978 2.73462 3.27020
C -2.38414 1.393634 .93111
H -2.66571 1.35115 5.98742
H - 1.341231 .724144 .90318

$1 \mathrm{~A}\left(C_{\mathrm{s}}, 0 \mathrm{nN}\right)$

Transition state $\left(\tilde{v}=-464.14 \mathrm{~cm}^{-1}\right)$

$E=-733.54805808$

$H=-733.24154155$

$G=-733.29243893$

$\mathrm{n}_{\text {imag }}=1$

C $0.44987-0.001192 .60877$

H -0.03453 -0.34948 3.53735

C $1.88718-0.440322 .69254$

H $2.59902-0.006263 .38256$

C $2.10344-1.486081 .89225$

H 3.02972 -2.04104 1.81798

C -0.07806 -0.84296 1.46400

C - $-1.11373-0.352440 .70382$

C $-1.11392-0.35285-0.70336$

C -0.07852 -0.84395 -1.46348

C $0.44877-0.00316-2.60905$

H -0.03625 -0.35204 -3.53715

C $1.88597-0.44262-2.69291$

C $2.10235-1.48816-1.89232$

H $2.59801-0.00829-3.38250$ 


$$
\begin{aligned}
& \text { H } 3.02889-2.04252-1.81703 \\
& \text { C - } 1.925250 .821251 .22616 \\
& \text { H -2.61707 0.499592.01350 } \\
& \text { C - } 1.925800 .82030-1.22615 \\
& \text { H -2.61765 } 0.49819-2.01331 \\
& \text { C - } 2.710471 .311150 .00001 \\
& \text { H -3.703230.857930.00023 } \\
& \text { H -2.85070 } 2.39435-0.00016 \\
& \text { C } 0.88978-1.80791-1.11763 \\
& \text { H } 0.62773-2.83589-0.94064 \\
& \text { C } 0.89070-1.806411 .11786 \\
& \text { H } 0.62904-2.834480 .94112 \\
& \text { C -0.97719 } 1.83470-1.82953 \\
& \text { H -1.23624 2.88557 -1.74359 } \\
& \text { C } 0.126871 .45836-2.45888 \\
& \text { H } 0.795822 .18741-2.90312 \\
& \text { C } 0.127731 .460252 .45828 \\
& \text { H } 0.796612 .189542 .90230 \\
& \text { C -0.97649 } 1.836171 .82897 \\
& \text { H -1.23569 2.88705 } 1.74309
\end{aligned}
$$

$1 \mathrm{~A}\left(C_{\mathrm{s}}, 2 \mathrm{nN}\right)$

Transition state $\left(\tilde{v}=-355.32 \mathrm{~cm}^{-1}\right)$

$E=-733.52534143$

$H=-733.21996016$

$G=-733.27050333$

$\mathrm{n}_{\text {imag }}=1$

C $0.20569-0.297082 .96169$

H -0.43629 -1.00875 3.50816

C $1.63309-0.639343 .32469$

H $1.98092-0.552474 .38418$ 


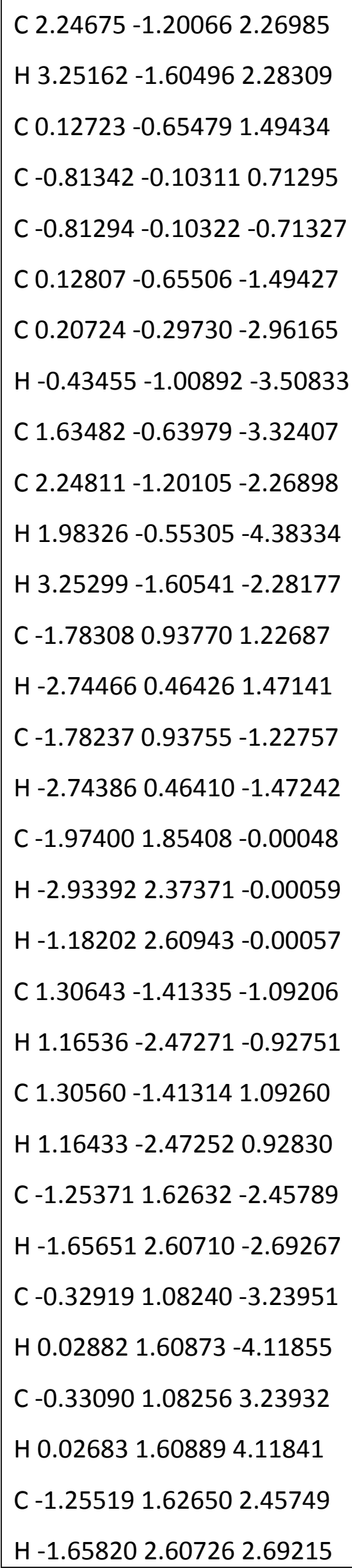


$1 \mathrm{~B}\left(C_{\mathrm{s}}, 0 \mathrm{nN}\right)$

Transition state ( $\left.\tilde{v}=-349.89 \mathrm{~cm}^{-1}\right)$

$E=-733.48858406$

$H=-733.18236058$

$G=-733.23384148$

$\mathrm{n}_{\text {imag }}=1$

C -3.19664 -0.46971 2.42274

H -3.53972 -1.51059 2.29176

C -2.27775 -0.18340 1.25289

H -1.31703 -0.66213 1.10352

C -2.872710.613590.35406

H -2.436420.88621 -0.59889

C -4.319010.45917 2.11280

C -5.351870 .461942 .94062$

C -6.618420.88554 2.57492

C -6.91503 1.32791 1.36325

C -8.34049 1.254050 .93760

H -8.63108 0.195610 .82120

C -8.23302 $1.81375-0.46601$

C -6.95199 $1.98243-0.82302$

H -9.07580 1.94000 -1.13554

H -6.63231 2.29550 -1.80920

C -5.38928 -0.23157 4.25531

H -5.64628 -1.29172 4.09347

C -7.616740.51461 3.61243

H -7.96036 -0.51664 3.42647

C -6.01969 1.858080 .36044

H -5.63930 2.842880 .59641

C -4.14029 1.228200 .90244

H -3.95715 2.27944 1.07997 
C -6.67505 0.437734 .87394
H -7.10424 -0.133685 .69767
H -6.44070 1.444325 .23085
C -3.96058 -0.216534 .78393
H -3.77021 -0.162625 .85042
C -2.93438 -0.326503 .92615
H -1.91550 -0.358874 .29642
C -8.82450 1.41364 3.37977
H -9.43697 1.737114 .21451
C -9.16236 1.760742 .12822
H -10.04445 2.366031 .94988

$1 \mathrm{~B}\left(C_{\mathrm{s}}, 2 \mathrm{nN}\right)$

Transition state $\left(\tilde{v}=-229.01 \mathrm{~cm}^{-1}\right)$

$E=-733.48431288$

$H=-733.17900167$

$G=-733.22975698$

$\mathrm{n}_{\text {imag }}=1$

C 0.546200 .000122 .91564

H $1.17497-0.867433 .17050$

C 1.312331 .239933 .35444

H 1.515251 .442304 .43729

C 1.744451 .927902 .27858

H 2.366552 .813142 .33267

C 0.489860 .178151 .43162

C $-0.19462-0.683440 .70366$

C -0.19472 -0.68337-0.70388

C $0.489690 .17833-1.43177$

C $0.546060 .00050-2.91583$

H $1.17483-0.86702-3.17073$

C $1.312361 .24036-3.35436$ 


$$
\begin{aligned}
& \text { C } 1.744381 .92816-2.27837 \\
& \text { H } 1.515491 .44293-4.43715 \\
& \text { H } 2.366412 .81344-2.33227 \\
& \text { C }-1.17530-1.672191 .23790 \\
& \text { H -0.71087 -2.65557 } 1.40167 \\
& \text { C -1.17564 -1.67198 -1.23803 } \\
& \text { H -0.71143 -2.65544 -1.40181 } \\
& \text { C } 1.032941 .47411-0.97455 \\
& \text { H } 0.207442 .17738-0.91639 \\
& \text { C } 1.033141 .473910 .97458 \\
& \text { H } 0.207582 .177110 .91649 \\
& \text { C - }-2.13482-1.793470 .00007 \\
& \text { H -2.71621 -2.71660 } 0.00009 \\
& \text { H -2.83121 -0.94976 } 0.00032 \\
& \text { C - } 1.64824-1.149642 .58498 \\
& \text { H -2.62923 -1.44356 2.94507 } \\
& \text { C }-0.86939-0.377643 .35220 \\
& \text { H -1.22234 -0.05191 4.32552 } \\
& \text { C - } 1.64851-1.14926-2.58511 \\
& \text { H -2.62963 -1.44286 -2.94513 } \\
& \text { C }-0.86961-0.37729-3.35236 \\
& \text { H - }-1.22268-0.05134-4.32556 \\
& \text { 1'A }\left(C_{s}, 0 \mathrm{nN}\right)
\end{aligned}
$$$$
\text { Transition state }\left(\tilde{v}=-491.18 \mathrm{~cm}^{-1}\right)
$$$$
E=-733.51250794
$$$$
H=-733.20575741
$$$$
G=-733.25665749
$$$$
\mathrm{n}_{\text {imag }}=1
$$

C $0.75993-0.000412 .28492$

H 1.439730 .547571 .60481 


\section{C $1.61177-1.167442 .70862$}

H 2.40574 -1.11188 3.44314

C $1.36667-2.210791 .90856$

H 1.91474 -3.14425 1.92484

C -0.24564-0.65125 1.37340

C -1.111590.152860.70787

C -1.11159 0.15286 -0.70787

C -0.24565 -0.65125 -1.37340

C $0.75993-0.00042-2.28491$

H 1.439730 .54755 -1.60480

C $1.61176-1.16746-2.70862$

C $1.36665-2.21081-1.90857$

H 2.40573 -1.11190 -3.44314

H 1.91471 -3.14427 -1.92485

C -1.44552 1.539361 .21525

H -2.53645 1.612851 .32582

C -1.44551 $1.53936-1.21525$

H -2.53644 1.61286 -1.32582

C $0.126611 .08114-3.11333$

C 0.126601 .081143 .11333

C -1.05007 2.424580.00000

H -1.53615 3.402160 .00000

H 0.032772 .583370 .00001

C $0.20130-1.95514-1.00711$

H -0.53936 -2.74270 -0.98880

C $0.20132-1.955131 .00710$

H -0.53933 -2.74269 0.98878

H 0.504281 .303744 .10559

H $0.504301 .30374-4.10558$

C -0.83786 $1.82283-2.56883$

H -1.25893 2.64731 -3.13569 
$C-0.837871 .822822 .56884$
H - 1.258952 .647303 .13570

$\mathbf{1}^{\prime} \mathrm{A}\left(C_{\mathrm{s}}, 1.8 \mathrm{nN}\right)$

Transition state ( $\tilde{v}=-195.85 \mathrm{~cm}^{-1}$ )

$E=-733.49279794$

$H=-733.18660366$

$G=-733.23691698$

$n_{\text {imag }}=1$

C $0.67818-0.000622 .62246$

H 1.529640 .608512 .26657

C $1.36896-1.238233 .18148$

H 1.69604 -1.33654 4.24322

C $1.54962-2.105012 .16761$

H $2.10181-3.033212 .25030$

C $0.02674-0.539691 .38736$

C -0.886250 .156710 .71726$

C - $0.886190 .15677-0.71730$

C $0.02683-0.53963-1.38739$

C $0.67823-0.00067-2.62258$

H $1.529720 .60842-2.26673$

C $1.36907-1.23834-3.18152$

C $1.54948-2.10516-2.16763$

H $1.69617-1.33674-4.24325$

H $2.10162-3.03338-2.25024$

C -0.19464 $1.01306-3.31121$

C -0.19476 1.012973.31113

C $0.65544-1.79392-0.94505$

H -0.07853 -2.59489-0.95530

C $0.65553-1.793910 .94500$

H -0.07834 -2.594960.95535 


$$
\begin{aligned}
& \text { H -0.00216 1.247934.35352 } \\
& \text { H -0.00197 1.24820 -4.35355 } \\
& \text { C -1.08202 1.73767 -2.62507 } \\
& \text { H -1.61414 2.53273 -3.13911 } \\
& \text { C -1.08214 } 1.737642 .62506 \\
& \text { H -1.61433 2.53260 } 3.13920 \\
& \text { C -1.50199 1.43375 1.20801 } \\
& \text { H -2.59388 } 1.295441 .23376 \\
& \text { C -1.50180 1.43383 -1.20799 } \\
& \text { H -2.59370 } 1.29560-1.23370 \\
& \text { C - } 1.232282 .387480 .00006 \\
& \text { H -1.86701 3.27559 -0.00001 } \\
& \text { H -0.18553 2.704920.00026 }
\end{aligned}
$$$$
\text { 1'B }^{\prime}\left(C_{\mathrm{s}}, 0 \mathrm{nN}\right)
$$$$
\text { Transition state }\left(\tilde{v}=-467.45 \mathrm{~cm}^{-1}\right)
$$$$
E=-733.47600091
$$$$
H=-733.17007961
$$$$
G=-733.22152461
$$$$
\mathrm{n}_{\text {imag }}=1
$$

C 0.732600 .199781 .52776

C - 0.218700 .692470 .70235

C - $0.217810 .69387-0.70207$

C $0.734230 .20201-1.52726$

C -1.656280 .852831 .15040$

H -2.09881 -0.14527 0.98182

C -1.65504 0.85443 -1.15069

H -2.09733 -0.14378 -0.98270

C - $0.920630 .48613-3.45028$

C - 0.923530 .482273 .45019

C - 2.274701 .678390 .00001 


$$
\begin{aligned}
& \text { H -3.36634 } 1.66587-0.00015 \\
& \text { H -1.92183 } 2.713370 .00031 \\
& \text { C } 1.88971-0.58288-1.19683 \\
& \text { H } 2.82824-0.16386-0.88799 \\
& \text { C } 1.88768-0.585741 .19727 \\
& \text { H } 2.82645-0.167180 .88880 \\
& \text { H -1.09514 } 0.507364 .52211 \\
& \text { H -1.09150 } 0.51229-4.52228 \\
& \text { C -1.81306 } 1.03178-2.62711 \\
& \text { H -2.72740 } 1.46422-3.02064 \\
& \text { C -1.81564 } 1.028352 .62692 \\
& \text { H -2.73048 } 1.459953 .02024 \\
& \text { C } 1.92638-1.61844-2.25624 \\
& \text { H } 2.61850-2.45053-2.24814 \\
& \text { C } 1.05749-1.34840-3.23924 \\
& \text { H } 0.94576-1.90781-4.15843 \\
& \text { C } 1.92346-1.622152 .25609 \\
& \text { H 2.61545 -2.45439 } 2.24794 \\
& \text { C } 1.05433-1.352503 .23899 \\
& \text { H } 0.94207-1.912684 .15770 \\
& \text { C } 0.428170 .00617-2.98983 \\
& \text { H } 1.132660 .68290-3.50972 \\
& \text { C } 0.425410 .002442 .99000 \\
& \text { H } 1.129630 .678643 .51069 \\
& \hline
\end{aligned}
$$$$
\mathbf{1}^{\prime} \mathrm{B}\left(C_{\mathrm{s}}, 2 \mathrm{nN}\right)
$$$$
\text { Transition state }\left(\tilde{v}=-341.5 \mathrm{~cm}^{-1}\right)
$$$$
E=-733.46441625
$$$$
H=-733.15949755
$$$$
G=-733.21027095
$$$$
\mathrm{n}_{\text {imag }}=1
$$ 
C 0.617670 .000091 .50213

C -0.390830 .346430 .71063$

C -0.39119 $0.34592-0.71052$

C $0.61710-0.00037-1.50232$

C -1.765330.72896 1.17466

H -2.37266 -0.18653 1.06717

C -1.76594 $0.72816-1.17432$

H -2.37341 -0.18724-1.06638

C -0.842920.59359 -3.46650

C -0.841560 .595283 .46660$

C -2.263191.620750.00002

H -3.34402 1.772950.00001

H -1.76360 2.59344-0.00013

C $1.78259-0.81445-1.13645$

H 2.72329 -0.31524 -0.94159

C $1.78257-0.814761 .13620$

H $2.72322-0.315350 .94133$

H -0.96840 0.72773 4.53738

H -0.96995 $0.72599-4.53726$

C -1.80595 $1.01648-2.64547$

H -2.70941 1.44844 -3.06535

C -1.804721.01806 2.64564

H -2.70817 1.449903 .06566

C $1.89761-1.67591-2.39964$

H 2.58618 -2.50934 -2.46769

C $1.20484-1.17442-3.44213$

H 1.25702 -1.46315 -4.52186

C $1.89805-1.675612 .39946$

H 2.58687 -2.50880 2.46745

C $1.20570-1.173563 .44195$

H 1.25826 -1.46208 4.52175 


$$
\begin{aligned}
& \text { C } 0.501490 .10120-2.99613 \\
& \text { H } 1.203630 .90012-3.30026 \\
& \text { C } 0.502650 .102272 .99604 \\
& \text { H } 1.204800 .901383 .29991 \\
& \hline \mathbf{2 A}\left(C_{s}, 0 \mathrm{nN}\right)
\end{aligned}
$$$$
\text { Transition state }\left(\tilde{v}=-438.61 \mathrm{~cm}^{-1}\right)
$$$$
E=-736.02036122
$$$$
H=-735.66475764
$$$$
G=-735.7169001
$$$$
\mathrm{n}_{\text {imag }}=1
$$

C 0.468000 .116062 .68653

H $0.13695-0.432883 .58366$

C 1.966980 .178872 .77648

H 2.492640 .790053 .49877

C $2.52004-0.710891 .94786$

H $3.57660-0.934561 .87534$

C $0.25415-0.781781 .48409$

C -0.87319 -0.624290 .70527$

C -0.87347 -0.62500 -0.70494

C $0.25375-0.78342-1.48362$

C $0.467750 .11317-2.68669$

H 0.13679 -0.43657 -3.58341

C $1.966770 .17574-2.77625$

C $2.51961-0.71374-1.94723$

H 2.492610 .78633 -3.49885

H 3.57608 -0.93772 -1.87436

C -2.092380.12713 1.21897

H -2.87959 -0.60211 1.44550

C -2.09281 $0.12622-1.21876$

H -2.87999 -0.60313 -1.44517 


$$
\begin{aligned}
& \text { C - } 1.829870 .97231-2.47725 \\
& \text { H -2.48080 } 1.85062-2.45785 \\
& \text { C -0.36382 } 1.38382-2.60372 \\
& \text { H -0.04876 } 1.97136-1.73477 \\
& \text { C -0.36354 } 1.386752 .60252 \\
& \text { H -0.04879 } 1.974051 .73320 \\
& \text { C - } 1.829480 .974362 .47686 \\
& \text { H -2.48044 } 1.852702 .45726 \\
& \text { C -2.51925 } 0.949540 .00001 \\
& \text { H -3.58406 } 1.194600 .00031
\end{aligned}
$$$$
\text { H -1.96273 } 1.89506-0.00012
$$$$
\text { C } 1.48319-1.38181-1.14326
$$$$
\text { H } 1.57033-2.42952-0.92294
$$$$
\text { C } 1.48388-1.379811 .14404
$$$$
\text { H } 1.57131-2.427570 .92437
$$$$
\text { H -2.10361 } 0.408083 .37226
$$$$
\text { H -0.21815 2.01214 } 3.48698
$$$$
\text { H -2.10435 } 0.40547-3.37223
$$$$
\text { H -0.21851 } 2.00828-3.48883
$$$$
2 \mathrm{~A}\left(C_{\mathrm{s}}, 2 \mathrm{nN}\right)
$$$$
\text { Transition state }\left(\tilde{v}=-343.99 \mathrm{~cm}^{-1}\right)
$$$$
E=-735.98728696
$$$$
H=-735.63272477
$$$$
G=-735.68577329
$$$$
\mathrm{n}_{\text {imag }}=1
$$

C $0.31766-0.190202 .93517$

H -0.09747 -1.05104 3.47826

C $1.77920-0.074003 .30633$

H 2.077130 .121554 .36707

C $2.55070-0.377642 .24950$ 


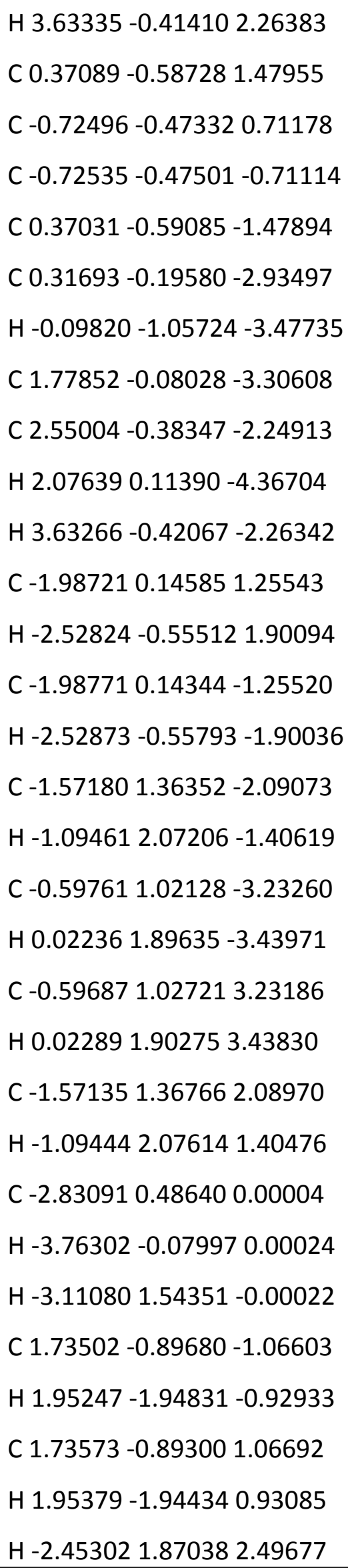




$$
\begin{aligned}
& \mathrm{H}-1.160760 .828074 .14636 \\
& \mathrm{H}-2.453521 .86571-2.49826 \\
& \mathrm{H}-1.161520 .82110-4.14693 \\
& \text { 2B }\left(C_{\mathrm{s}}, 0 \mathrm{nN}\right) \\
& \text { Transition state }\left(\tilde{v}=-389.70 \mathrm{~cm}^{-1}\right) \\
& E=-735.97409192 \\
& H=-735.61856046 \\
& G=-735.67192532 \\
& \mathrm{n}_{\text {imag }}=1
\end{aligned}
$$

C 0.670400 .000022 .67023

H $1.33740-0.836782 .40330$

C 1.571781 .141373 .07778

H 2.180901 .146403 .97405

C 1.619752 .077892 .12055

H 2.252772 .956022 .15508

C 0.076480 .548501 .41823

C - $0.64878-0.310140 .69638$

C -0.64878 -0.31014-0.69638

C $0.076480 .54850-1.41823$

C $0.670400 .00002-2.67023$

H $1.33740-0.83678-2.40330$

C $1.571781 .14137-3.07778$

C $1.619752 .07789-2.12055$

H 2.18090 1.14640 -3.97405

H $2.252762 .95602-2.15508$

C -0.96439-1.67868 1.19993

H -0.01990 -2.24765 1.21329

C -0.96439 -1.67868 -1.19993

H -0.01990 -2.24765 -1.21329

C $0.608051 .82784-1.03717$ 


$$
\begin{aligned}
& \text { H -0.09972 2.64069 -0.95021 } \\
& \text { C } 0.608051 .827841 .03717 \\
& \text { H -0.09972 2.640690.95021 } \\
& \text { C -1.78333 -2.253180.00000 } \\
& \text { H -1.84872 -3.34216 } 0.00000 \\
& \text { H -2.79919 -1.84438 } 0.00000 \\
& \text { C -1.42776-1.55364-2.66155 } \\
& \text { H -1.49442 -2.53559-3.13444 } \\
& \text { H -2.43329 -1.12620 -2.67661 } \\
& \text { C -0.46974 -0.62300 -3.51337 } \\
& \text { H - } 1.060820 .18076-3.95710 \\
& \text { H -0.04406 -1.19673 -4.33944 } \\
& \text { C -1.42776-1.55364 2.66155 } \\
& \text { H -2.43329 -1.12620 2.67661 } \\
& \text { H -1.49442 -2.53559 3.13444 } \\
& \text { C -0.46974 -0.62300 } 3.51337 \\
& \text { H -0.04406 -1.19673 4.33944 } \\
& \text { H -1.060820.18076 } 3.95710 \\
& \text { 2B }\left(C_{s}, 2 \mathrm{nN}\right)
\end{aligned}
$$$$
\text { Transition state }\left(\tilde{v}=-183.88 \mathrm{~cm}^{-1}\right)
$$$$
E=-735.96346435
$$$$
H=-735.60898994
$$$$
G=-735.66280171
$$$$
\mathrm{n}_{\text {imag }}=1
$$

C $0.57316-0.002362 .88218$

H $1.12507-0.941723 .03584$

C 1.473671 .145313 .31404

H 1.737731 .303504 .39126

C 1.893791 .845142 .23978

H 2.583852 .679102 .29102 


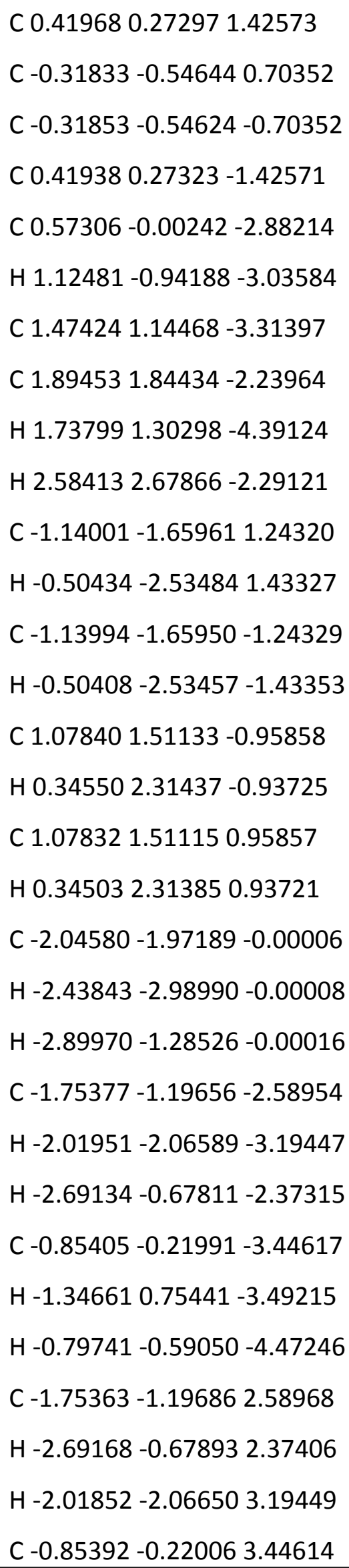




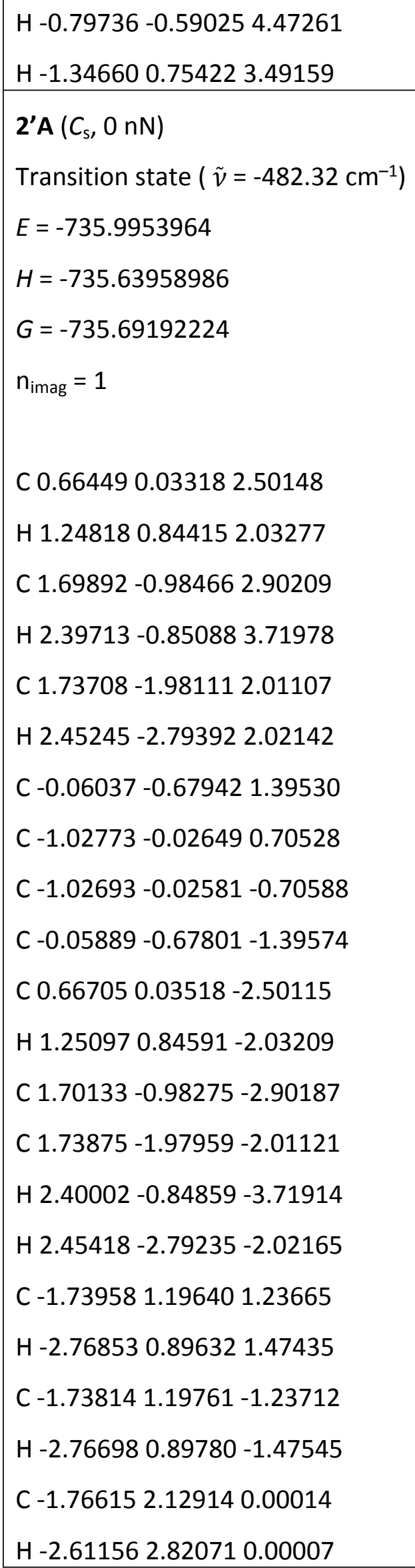




$$
\begin{aligned}
& \text { H -0.84987 2.730420.00048 } \\
& \text { C } 0.61742-1.87902-1.02758 \\
& \text { H } 0.01688-2.77433-0.95798 \\
& \text { C } 0.61636-1.880101 .02706 \\
& \text { H } 0.01593-2.775570 .95686 \\
& \text { C -1.08991 1.77550-2.51354 } \\
& \text { H -1.84353 2.29775 -3.10789 } \\
& \text { H -0.35604 2.53351 -2.21771 } \\
& \text { C }-0.349450 .74142-3.39363 \\
& \text { H } 0.133911 .26174-4.22489 \\
& \text { H -1.04733 } 0.01592-3.82154 \\
& \text { C -1.09267 1.77322 2.51385 } \\
& \text { H -0.35886 2.531372.21854 } \\
& \text { H -1.84676 2.294913.10815 } \\
& \text { C - } 0.352800 .738583 .39386 \\
& \text { H -1.050760.01270 } 3.82125 \\
& \text { H } 0.129961 .258554 .22561
\end{aligned}
$$

2'A $\left(C_{s}, 2 \mathrm{nN}\right)$

Transition state ( $\left.\tilde{v}=-201.57 \mathrm{~cm}^{-1}\right)$

$E=-735.97984405$

$H=-735.62514626$

$G=-735.67670453$

$\mathrm{n}_{\text {imag }}=1$

C 0.623710 .000942 .75362

H 1.329050 .831432 .58214

C $1.49579-1.143423 .24238$

H 1.80853 -1.24088 4.31343

C $1.84137-1.910292 .19059$

H $2.53040-2.744802 .24413$

C $0.17600-0.494501 .40831$ 
C -0.77817 0.124220.71631

C -0.77899 $0.12365-0.71581$

C $0.17448-0.49577-1.40804$

C $0.62100-0.00125-2.75389$

H $1.326260 .82926-2.58277$

C $1.49273-1.14584-3.24271$

C $1.83889-1.91235-2.19085$

H 1.80478 -1.24384 -4.31392

H 2.52772 -2.74703 -2.24442

C -1.622171.26027 1.22520

H -2.642950.875401.35986

C -1.62346 1.25932-1.22472

H -2.64431 $0.87434-1.35891$

C -1.63930 2.21409-0.00001

H -2.48741 2.90220 -0.00001

H -0.71992 2.81098 -0.00022

C $0.94370-1.66765-0.95256$

H 0.29640 -2.54011 -0.94223

C $0.94508-1.666480 .95282$

H 0.29795 -2.53897 0.94304

C -1.11870 $1.77153-2.58078$

H -1.91241 2.30811 -3.10629

H -0.31213 $2.49456-2.41387$

C $-0.564460 .63533-3.47786$

H -0.26282 $1.05065-4.44320$

H -1.34159-0.11122 -3.67088

C -1.11622 1.77358 2.58061

H -0.30968 2.49669 2.41342

H -1.90964 2.31050 3.10619

C -0.561290 .637913 .47783$

H -1.33826 -0.10856 3.67124 


$$
\begin{aligned}
& \text { H -0.25906 } 1.053664 .44285 \\
& \mathbf{2}^{\prime} \mathrm{B}\left(C_{\mathrm{s}}, 0 \mathrm{nN}\right) \\
& \text { Transition state }\left(\tilde{v}=-447.20 \mathrm{~cm}^{-1}\right) \\
& E=-735.97739248 \\
& H=-735.6222599 \\
& G=-735.6745276 \\
& \mathrm{n}_{\text {imag }}=1 \\
& \text { C } 0.40483-0.003102 .96602 \\
& \text { H } 0.531920 .931463 .53949 \\
& \text { C } 1.68602-0.763173 .19890 \\
& \text { H } 1.90110 \text {-1.31322 } 4.10616 \\
& \text { C } 2.55738-0.510602 .21505 \\
& \text { H } 3.58982-0.835482 .19596 \\
& \text { C } 0.568410 .412071 .52092 \\
& \text { C - } 0.508430 .475750 .70201 \\
& \text { C - } 0.508010 .47693-0.70159 \\
& \text { C } 0.569140 .41657-1.52068 \\
& \text { C } 0.405600 .00203-2.96599 \\
& \text { H } 0.531740 .93678-3.53957 \\
& \text { C } 1.68705-0.75721-3.19948 \\
& \text { C } 2.55850-0.50527-2.21566 \\
& \text { H } 1.90052-1.31029-4.10519 \\
& \text { H } 3.58964-0.83417-2.19437 \\
& \text { C -1.89476 0.07327 1.15754 } \\
& \text { H -1.93282 -1.021361.02786 } \\
& \text { C -1.89413 0.07519-1.15780 } \\
& \text { H -1.93245 -1.01944 -1.02866 } \\
& \text { C -2.77366 0.59424 -0.00005 } \\
& \text { H -3.78163 0.17423 -0.00047 } \\
& \text { H -2.83889 } 1.686850 .00014
\end{aligned}
$$




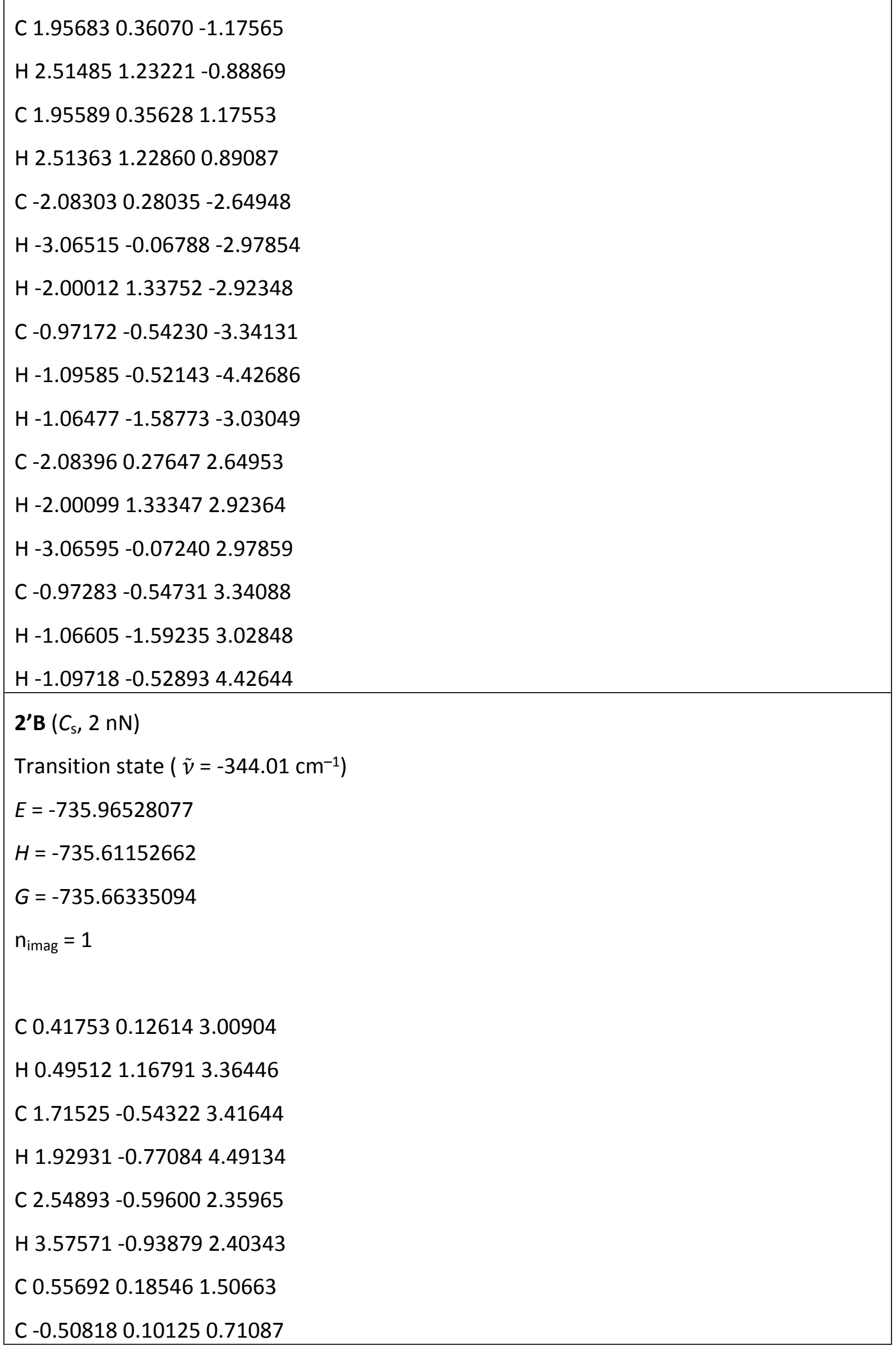




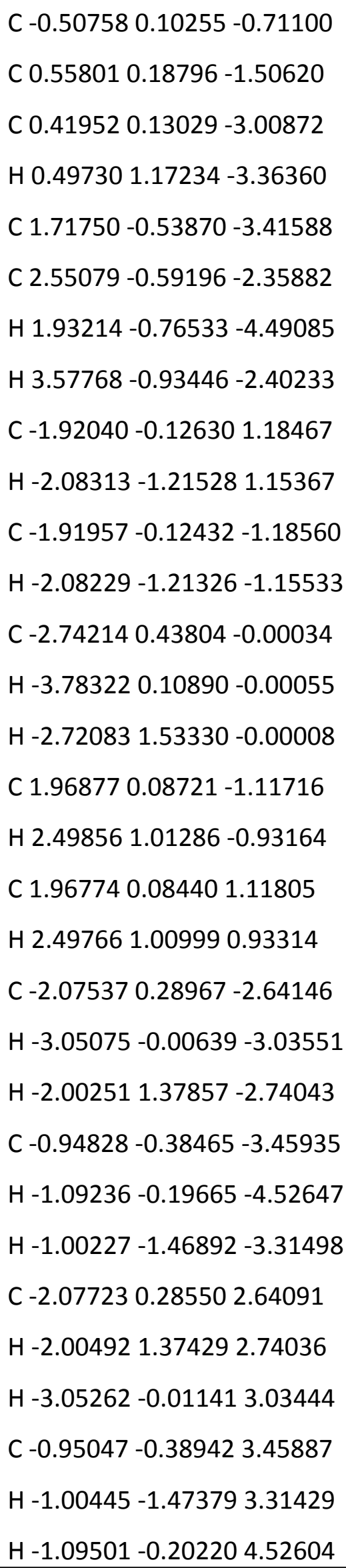


$3 \mathrm{~A}\left(C_{\mathrm{s}}, 0 \mathrm{nN}\right)$

Transition state $\left(\tilde{v}=-534.39 \mathrm{~cm}^{-1}\right)$

$E=-812.18183802$

$H=-811.81415847$

$G=-811.87009186$

$\mathrm{n}_{\text {imag }}=1$

C 0.704620 .002062 .57038

H 0.163590 .234083 .49822

C 2.153050 .387472 .71851

H $2.81272-0.086313 .43489$

C 2.485881 .362781 .87492

H 3.461681 .824691 .79622

C 0.247420 .914931 .44844

C -0.891780 .695310 .70638$

C -0.892020.69564-0.70622

C $0.247050 .91512-1.44844$

C $0.704170 .00219-2.57038$

H $0.163000 .23413-3.49814$

C $2.152540 .38787-2.71881$

C $2.485481 .36296-1.87501$

H $2.81220-0.08592-3.43519$

H $3.461321 .82470-1.79606$

C -2.143360.01052 1.22590

H -2.579210.60448 2.03756

C -2.14355 0.01079-1.22560

H -2.57956 $0.60475-2.03715$

C $0.54088-1.49133-2.26674$

H $1.07330-1.71258-1.33400$

C $0.54144-1.491422 .26661$

H $1.07427-1.712431 .33405$ 


$$
\begin{aligned}
& \text { C -3.07452 0.062260.00021 } \\
& \text { H -3.61181 1.012840.00030 } \\
& \text { H -3.81812 -0.73680 } 0.00017 \\
& \text { C } 1.314371 .76249-1.06707 \\
& \text { H } 1.127282 .81576-0.94111 \\
& \text { C } 1.314741 .762341 .06692 \\
& \text { H } 1.127612 .815610 .94133 \\
& \text { H } 1.05925 \text {-2.06026 } 3.04447 \\
& \text { H } 1.05879 \text {-2.06008 -3.04459 } \\
& \text { C -1.96283 -1.39327 } 1.74861 \\
& \text { H -2.88805 -1.96444 } 1.78508 \\
& \text { C -0.86757-2.00972 2.17363 } \\
& \text { H -0.98966 -3.041112.49369 } \\
& \text { C -1.96329 -1.39290 -1.74858 } \\
& \text { H - 2.88856 -1.96398 -1.78477 } \\
& \text { C }-0.86826-2.00932-2.17418 \\
& \text { H -0.99064 -3.04063 -2.49443 }
\end{aligned}
$$$$
3 \mathrm{~A}\left(C_{\mathrm{s}}, 2 \mathrm{nN}\right)
$$$$
\text { Transition state }\left(\tilde{v}=-353.10 \mathrm{~cm}^{-1}\right)
$$$$
E=-812.1580902
$$$$
H=-811.79164611
$$$$
G=-811.84700887
$$$$
\mathrm{n}_{\text {imag }}=1
$$

C 0.555560 .001232 .90230

H $0.10546-0.775323 .53511$

C 1.999080 .242533 .28668

H 2.268410 .473884 .34810

C 2.795730 .083452 .21941

H 3.871690 .209492 .22441

C $0.65903-0.501351 .47513$ 


$$
\begin{aligned}
& \text { C }-0.41343-0.799270 .71714 \\
& \text { C }-0.41310-0.79948-0.71694 \\
& \text { C } 0.65978-0.50194-1.47461 \\
& \text { C } 0.556820 .00012-2.90197 \\
& \text { H } 0.10694-0.77667-3.53464 \\
& \text { C } 2.000430 .24128-3.28598 \\
& \text { C } 2.796880 .08238-2.21855 \\
& \text { H } 2.270050 .47224-4.34738 \\
& \text { H } 3.872890 .20813-2.22340 \\
& \text { C }-1.83417-0.889621 .23842 \\
& \text { H }-1.89213-1.667592 .00858 \\
& \text { C }-1.83365-0.89004-1.23867 \\
& \text { H }-1.89145-1.66814-2.00873 \\
& \text { C }-0.263571 .29093-3.04011 \\
& \text { H } 0.255012 .08102-2.48334 \\
& \text { C }-0.265001 .292173 .03965
\end{aligned}
$$$$
\text { H } 0.253462 .082182 .48263
$$$$
\text { C - } 2.62983-1.34883-0.00019
$$$$
\text { H -2.68972 -2.43879 -0.00027 }
$$$$
\text { H -3.65425 -0.97131-0.00022 }
$$$$
\text { C } 2.05412-0.53639-1.04077
$$$$
\text { H } 2.43021-1.54379-0.91838
$$$$
\text { C } 2.05341-0.535681 .04156
$$$$
\text { H } 2.42947 \text {-1.54307 } 0.91934
$$$$
\text { H -0.23913 1.612004.08742 }
$$$$
\text { H -0.23744 } 1.61028-4.08804
$$$$
\text { C - } 2.353980 .390711 .84958
$$$$
\text { H -3.407370.58832 } 1.66543
$$$$
\text { C -1.70191 1.27170 2.59814 }
$$$$
\text { H -2.27351 2.13122 2.94015 }
$$$$
\text { C - } 2.353030 .39006-1.85040
$$ 


$$
\begin{aligned}
& \mathrm{H}-3.406360 .58786-1.66630 \\
& \mathrm{C}-1.700611 .27072-2.59902 \\
& \mathrm{H}-2.272002 .13026-2.94130 \\
& \text { 3B }\left(C_{\mathrm{s}}, 0 \mathrm{nN}\right) \\
& \text { Transition state }\left(\tilde{v}=-467.27 \mathrm{~cm}^{-1}\right) \\
& E=-812.17850448 \\
& H=-811.81115533 \\
& G=-811.86730758 \\
& \mathrm{n}_{\text {imag }}=1
\end{aligned}
$$

C 0.890070 .000252 .73433

H 1.26549 -1.02103 2.58769

C 2.031480 .955002 .95941

H 2.730200 .858843 .78142

C 2.037161 .923372 .04115

H 2.740822 .745022 .00040

C 0.281820 .538311 .46264

C - $0.54800-0.231370 .70312$

C $-0.54862-0.23085-0.70295$

C $0.280510 .53948-1.46262$

C $0.888020 .00191-2.73464$

H 1.26335 -1.01939 -2.58817

C $2.029280 .95673-2.96007$

C $2.035391 .92493-2.04168$

H 2.72771 $0.86064-3.78232$

H $2.739162 .74648-2.00089$

C -1.15480 -1.52715 1.19710

H -0.33793 -2.26397 1.27063

C -1.15585 -1.52628 -1.19716

H -0.33910 -2.26313 -1.27101

C - $1.82859-1.45539-2.54215$ 


$$
\begin{aligned}
& \text { C }-0.14352-0.02481-3.87176 \\
& \text { H -0.44264 } 1.00660-4.09383 \\
& \text { C -0.14087-0.02717 } 3.87197 \\
& \text { H -0.44005 1.00407 4.09449 } \\
& \text { C }-1.82648-1.457292 .54250 \\
& \text { C - } 2.02174-1.947470 .00013 \\
& \text { H -2.26805 -3.010990.00006 } \\
& \text { H -2.95682 -1.37700 } 0.00042 \\
& \text { C } 0.901941 .76321-1.10010 \\
& \text { H } 0.297342 .63553-0.92140 \\
& \text { C } 0.903101 .762121 .10008 \\
& \text { H } 0.298412 .634430 .92204 \\
& \text { H -2.76781 -1.99555 2.62297 } \\
& \text { H } 0.34048-0.398004 .78411 \\
& \text { H -2.76997 -1.99362 -2.62264 } \\
& \text { H } 0.33731-0.39530-4.78426 \\
& \text { C -1.39229 -0.83787 -3.63668 } \\
& \text { H -2.02808 -0.90933 -4.51611 } \\
& \text { C - }-1.38970-0.840283 .63712 \\
& \text { H -2.02533 -0.91200 4.51664 }
\end{aligned}
$$$$
\text { 3B }\left(C_{\mathrm{s}}, 2 \mathrm{nN}\right)
$$$$
\text { Transition state }\left(\tilde{v}=-357.56 \mathrm{~cm}^{-1}\right)
$$$$
E=-812.16063803
$$$$
H=-811.79440556
$$$$
G=-811.85015492
$$$$
\mathrm{n}_{\text {imag }}=1
$$

C $0.75347-0.000442 .95784$

H 0.97338 -1.06552 3.11006

C 1.949810 .847823 .31889

H 2.294560 .902084 .38279 

C 2.373981 .534622 .24731
H 3.197532 .238292 .24654
C 0.536920 .311351 .49021
C - $0.32398-0.376310 .71729$
C -0.32349-0.37677-0.71722
C $0.537900 .31040-1.49002$
C $0.75500-0.00199-2.95752$
H 0.97499 -1.06712 -3.10951
C $1.951520 .84601-3.31851$
C $2.375601 .53298-2.24699$
H $2.296630 .89985-4.38231$
H $3.199252 .23653-2.24618$
C -1.23572 -1.48001 1.21707
H -0.62174 -2.37978 1.37922
C -1.23511 -1.48069-1.21702
H -0.62108 -2.38049 -1.37895
C - $2.01893-1.21862-2.47508$
C -0.47847 0.34624 -3.79789
H -0.70645 $1.41131-3.66918$
C - 0.480320 .348103 .79776
H -0.70829 1.413193 .66880
C -2.02020 -1.21717 2.47469
C $-2.13522-1.73860-0.00008$
H - $2.57771-2.736750 .00000$
H -2.95195 -1.00864 -0.00017
C $1.426051 .38821-1.06649$
H $0.930982 .33706-0.91260$
C 1.424851 .389261 .06660
H 0.929792 .338090 .91229
H -2.97524 -1.73706 2.51278
H -0.22681 0.226894 .85702 


$$
\begin{aligned}
& \mathrm{H}-2.97389-1.73862-2.51333 \\
& \mathrm{H}-0.224460 .22465-4.85701 \\
& \mathrm{C}-1.71553-0.46188-3.52351 \\
& \mathrm{H}-2.46117-0.41111-4.31320 \\
& \mathrm{C}-1.71721-0.460203 .52310 \\
& \mathrm{H}-2.46313-0.409174 .31253 \\
& \text { 3'A }\left(C_{\mathrm{s}}, 0 \mathrm{nN}\right) \\
& \text { Transition state }\left(\tilde{v}=-535.26 \mathrm{~cm}^{-1}\right) \\
& E=-812.17493772 \\
& H=-811.807254 \\
& G=-811.86361694 \\
& \mathrm{n}_{\text {imag }}=1
\end{aligned}
$$

C 0.934150 .000742 .51204

H 1.298500 .957992 .11571

C $2.10690-0.922882 .69030$

H $2.90297-0.729803 .39917$

C $2.02968-1.967191 .86623$

H 2.75104 -2.77124 1.79648

C $0.13098-0.702001 .43752$

C - $0.85941-0.083270 .70782$

C $-0.85941-0.08327-0.70782$

C $0.13098-0.70200-1.43752$

C $0.934150 .00073-2.51204$

H $1.298500 .95798-2.11570$

C $2.10690-0.92289-2.69030$

C $2.02967-1.96720-1.86623$

H $2.90297-0.72981$-3.39917

H $2.75103-2.77126-1.79649$

C -1.80222 0.995811 .20153

H -2.78549 0.50122 1.27910 


$$
\begin{aligned}
& \text { C }-1.802220 .99581-1.20153 \\
& \text { H -2.78549 } 0.50123-1.27910 \\
& \text { C -1.89321 } 1.946590 .00000 \\
& \text { H -2.801772.553610.00000 } \\
& \text { H -1.02959 2.619760.00000 } \\
& \text { C } 0.79108-1.89500-1.06095 \\
& \text { H } 0.21318-2.79767-0.94847 \\
& \text { C } 0.79109-1.894991 .06095 \\
& \text { H } 0.21319-2.797660 .94847 \\
& \text { C -1.62427 } 1.68182-2.52661 \\
& \text { H -2.29847 2.52987 -2.63428 } \\
& \text { C -1.624281.68182 2.52661 } \\
& \text { H -2.29848 } 2.529872 .63429 \\
& \text { С } 0.148990 .32139-3.78329 \\
& \text { H -0.32090 -0.59296-4.16212 } \\
& \text { H } 0.848620 .64785 \text {-4.55839 } \\
& \text { C -0.88105 } 1.39946-3.59209 \\
& \text { H -1.02645 2.04517 -4.45414 } \\
& \text { C } 0.148990 .321393 .78329 \\
& \text { H -0.32090 -0.59296 4.16212 } \\
& \text { H } 0.848620 .647854 .55839 \\
& \text { C -0.88105 1.39946 } 3.59209 \\
& \text { H -1.026462.045174.45414 }
\end{aligned}
$$$$
\text { 3'A }\left(C_{\mathrm{s}}, 2 \mathrm{nN}\right)
$$$$
\text { Transition state }\left(\tilde{v}=-379.89 \mathrm{~cm}^{-1}\right)
$$$$
E=-812.14941532
$$$$
H=-811.78331431
$$$$
G=-811.83923523
$$$$
\mathrm{n}_{\text {imag }}=1
$$ 


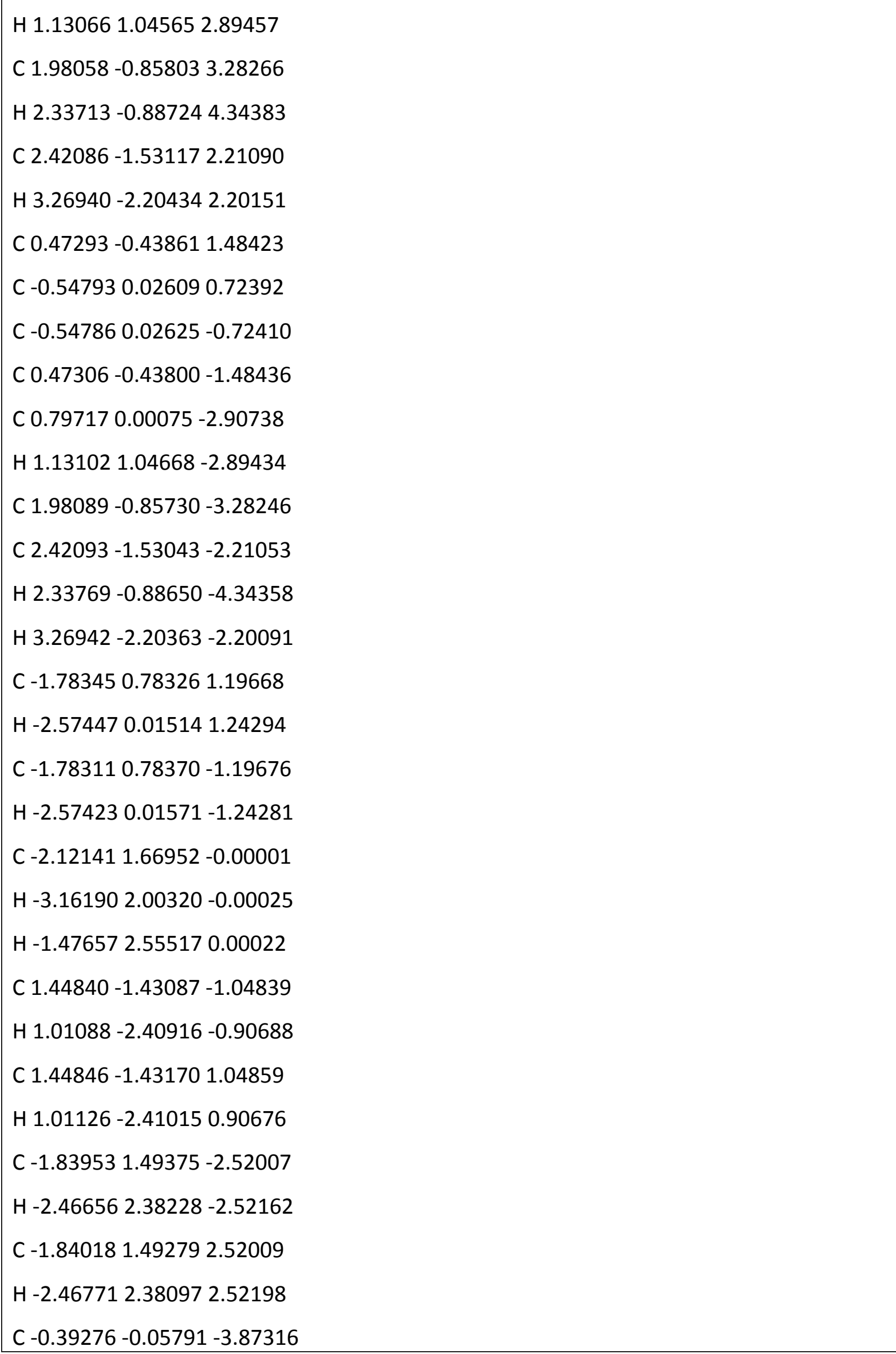




$$
\begin{aligned}
& \text { H }-0.92613-1.00546-3.74086 \\
& \text { H -0.01689 }-0.05296-4.89931 \\
& \text { C -1.31331 } 1.11338-3.67609 \\
& \text { H -1.52476 } 1.72207-4.55014 \\
& \text { C -0.39361 }-0.059443 .87272 \\
& \text { H -0.92661 -1.00716 3.73961 } \\
& \text { H -0.01857 -0.05504 } 4.89914 \\
& \text { C -1.31403 } 1.111943 .67593 \\
& \text { H }-1.525741 .720094 .55026
\end{aligned}
$$$$
\text { 3'B }\left(C_{s}, 0 \mathrm{nN}\right)
$$$$
\text { Transition state }\left(\tilde{v}=-425.44 \mathrm{~cm}^{-1}\right)
$$$$
E=-812.15429639
$$$$
H=-811.78751607
$$$$
G=-811.84314009
$$$$
\mathrm{n}_{\text {imag }}=1
$$

C $0.77978-0.055421 .52816$

C -0.072180 .659620 .70889$

C $-0.072890 .65910-0.70903$

C $0.77814-0.05664-1.52852$

C - 1.449741 .154741 .16259

H -2.101340.26761 1.10142

C -1.45077 1.15394 -1.16226

H -2.10245 0.26684 -1.10082

C -1.88387 2.036480 .00009

H -2.95475 2.249640.00026

H -1.33297 2.98337 -0.00012

C $2.01239-0.65390-1.17868$

H $2.85724-0.07314-0.86855$

C $2.01404-0.652661 .17805$

H $2.85877-0.071810 .86752$ 


$$
\begin{aligned}
& \text { C -1.60567 } 1.66724-2.55990 \\
& \text { H -1.87624 2.70829 -2.70785 } \\
& \text { C -1.60348 } 1.668912 .56012 \\
& \text { H -1.87377 2.71009 2.70792 } \\
& \text { C -1.00170 -0.57498 -3.41776 } \\
& \text { H -1.63542 -1.12173 -2.71178 } \\
& \text { H - } 1.05178-1.11208-4.36714 \\
& \text { C - }-1.474130 .84756-3.59460 \\
& \text { H -1.62135 } 1.21641-4.60466 \\
& \text { C -0.99883 -0.57279 } 3.41814 \\
& \text { H -1.63258 -1.11926 2.71208 } \\
& \text { H -1.04853 -1.10968 4.36764 } \\
& \text { C -1.471530.84963 } 3.59504 \\
& \text { H -1.61806 1.21890 4.60506 } \\
& \text { C } 2.23563-1.77980-2.09549 \\
& \text { H } 3.06506 \text {-2.46953 -2.01103 } \\
& \text { C } 1.31034-1.77849-3.05713 \\
& \text { H } 1.25463-2.45685 \text {-3.89871 } \\
& \text { C 2.23794-1.77812 2.09507 } \\
& \text { H } 3.06765 \text {-2.46752 } 2.01058 \\
& \text { C } 1.31301-1.776463 .05701 \\
& \text { H } 1.25791-2.454493 .89887 \\
& \text { C } 0.44726-0.55258-2.93373 \\
& \text { H } 0.954680 .17508-3.58971 \\
& \text { C } 0.44983-0.550642 .93364 \\
& \text { H } 0.957210 .177433 .58929
\end{aligned}
$$$$
\mathbf{3}^{\prime} \mathrm{B}\left(C_{\mathrm{s}}, 2 \mathrm{nN}\right)
$$$$
\text { Transition state }\left(\tilde{v}=-406.16 \mathrm{~cm}^{-1}\right)
$$$$
E=-812.14336226
$$$$
H=-811.77784989
$$$$
G=-811.83326076
$$ 


$$
\mathrm{n}_{\text {imag }}=1
$$

C -4.019960.84253 1.64397

C -4.78601 1.822782 .26388

C -6.13140 2.215621 .98731

C -6.90592 1.791520 .93524

C -4.50072 2.29593 3.70292

H -4.88827 1.491394 .34675

C -6.71465 2.840473 .25129

H -6.98993 1.987823 .89499

C -5.47876 3.446463 .89790

H -5.62123 3.706924 .94874

H -5.15231 4.33804 3.35152

C -6.35426 $1.12851-0.19476$

H -5.56218 $1.56084-0.77595$

C -4.19146 0.170620.37954

H -3.81087 0.53088 -0.59224

C -7.95816 3.669693 .13026

H -7.91981 4.70006 3.47145

C -3.08002 2.500104 .12158

H -2.72875 3.506334 .32830

C -9.21374 1.764902 .12335

H -8.84022 1.014982 .82851

H -10.25817 1.520381 .92077

C -9.10376 3.159272 .69498

H -9.98957 3.786152 .66606

C -2.77349 0.072283 .90073

H -3.70141 -0.18893 4.41931

H - $2.03617-0.682064 .18438$

C -2.29269 1.446834 .28739

H -1.26777 1.565874 .62316 


$$
\begin{aligned}
& \text { C -7.43749 0.35743 -0.80911 } \\
& \text { H -7.30997 -0.29891 -1.65978 } \\
& \text { C -8.59566 } 0.61565-0.19031 \\
& \text { H -9.56705 } 0.22420-0.46348 \\
& \text { C -3.75469-1.22467 0.59086 } \\
& \text { H -3.89217 -2.00393 -0.14783 } \\
& \text { C -3.09332 -1.33994 } 1.74027 \\
& \text { H -2.59274 -2.22406 2.11373 } \\
& \text { C -8.40992 1.714970.82508 } \\
& \text { H -8.69388 2.623680.26896 } \\
& \text { C - } 2.956520 .010172 .38563 \\
& \text { H -2.007340.39631 } 1.97581 \\
& \text { 4A }\left(C_{s}, 0 \mathrm{nN}\right)
\end{aligned}
$$$$
\text { Transition state }\left(\tilde{v}=-501.06 \mathrm{~cm}^{-1}\right)
$$$$
E=-994.82275228
$$$$
H=-994.46558062
$$$$
G=-994.56246731
$$$$
\mathrm{n}_{\text {imag }}=1
$$$$
\text { C - } 0.74465-0.567502 .59790
$$$$
\text { H -0.81417 -0.02103 } 3.54985
$$$$
\text { C -1.82350 -1.61499 2.63598 }
$$$$
\text { H -1.78632 -2.46335 } 3.30689
$$$$
\text { C -2.83466 -1.28515 } 1.83306
$$$$
\text { H -3.76591 -1.82606 1.73007 }
$$$$
\text { C - } 1.212760 .338141 .47357
$$$$
\text { C - } 0.344431 .094320 .70701
$$$$
\text { C - }-0.345981 .09601-0.70581
$$$$
\text { C -1.21687 0.34210 -1.47187 }
$$$$
\text { C }-0.75108-0.56174-2.59777
$$$$
\text { H -0.82175 -0.01434 -3.54918 }
$$ 
C - $1.83027-1.60888-2.63503$
C - $2.84076-1.27931-1.83123$
H - $1.79423-2.45671-3.30645$
H -3.77240 - $1.81951-1.72758$

C 0.957971 .708191 .19975

H 0.747962 .773901 .35555

C $0.955431 .71074-1.19966$

H $0.745182 .77657-1.35445$

C $0.68526-1.06431-2.44983$

H 0.87853 -1.32837-1.40576

C $0.69186-1.070402 .44788$

H $0.88518-1.334511 .40365$

C $1.894331 .57080-0.00058$

H $2.708722 .29792-0.00075$

H $2.331710 .56780-0.00112$

C -2.51947 -0.04231-1.10182

H -3.28417 $0.69436-0.93154$

C -2.51529-0.04699 1.10423

H -3.280220.689510.93548

H 0.87318 -1.95499 3.05641

H 0.86570 -1.94826 -3.05939

N $1.512961 .25492-2.45816$

H $2.183071 .88431-2.87272$

N 1.518051 .249912 .45673

H 2.188841 .878712 .87110

C $1.66754-0.01941-2.93231$

C $1.67362-0.025012 .93019$

O $2.49358-0.26258-3.78733$

O $2.49985-0.268343 .78502$

$4 \mathrm{~A}\left(C_{\mathrm{s}}, 2 \mathrm{nN}\right)$

Transition state $\left(\tilde{v}=-394.00 \mathrm{~cm}^{-1}\right)$ 


$$
\begin{aligned}
& E=-994.79310467 \\
& H=-994.43725153 \\
& G=-994.49709114 \\
& n_{\text {imag }}=1
\end{aligned}
$$

C -0.75983 -0.42426 2.95361

H -0.950630.46684 3.56622

C -1.77021 -1.48745 3.30939

H - 1.85758 -1.83533 4.36858

C -2.52047 -1.78864 2.24071

H -3.33662 -2.50004 2.22983

C -1.07299 -0.10315 1.50251

C - 0.289800 .674580 .72187

C - $0.288720 .67472-0.72188$

C -1.07074-0.10289-1.50348

C - $0.75614-0.42376-2.95430$

H -0.94625 $0.46746-3.56700$

C $-1.76638-1.48678-3.31110$

C - $2.51724-1.78817-2.24288$

H -1.85262 -1.83463 -4.37049

H -3.33307 -2.49984 -2.23262

C 0.832041 .584601 .19567

H 0.382562 .584971 .27750

C $0.833541 .58494-1.19454$

H $0.384122 .58534-1.27632$

C $0.69145-0.86931-3.17628$

H 0.99098 -1.54597 -2.37082

C $0.68739-0.869853 .17705$

H 0.98685 -1.54664 2.37172

C 1.783771 .600480 .00097

H 2.458782 .459260 .00126 


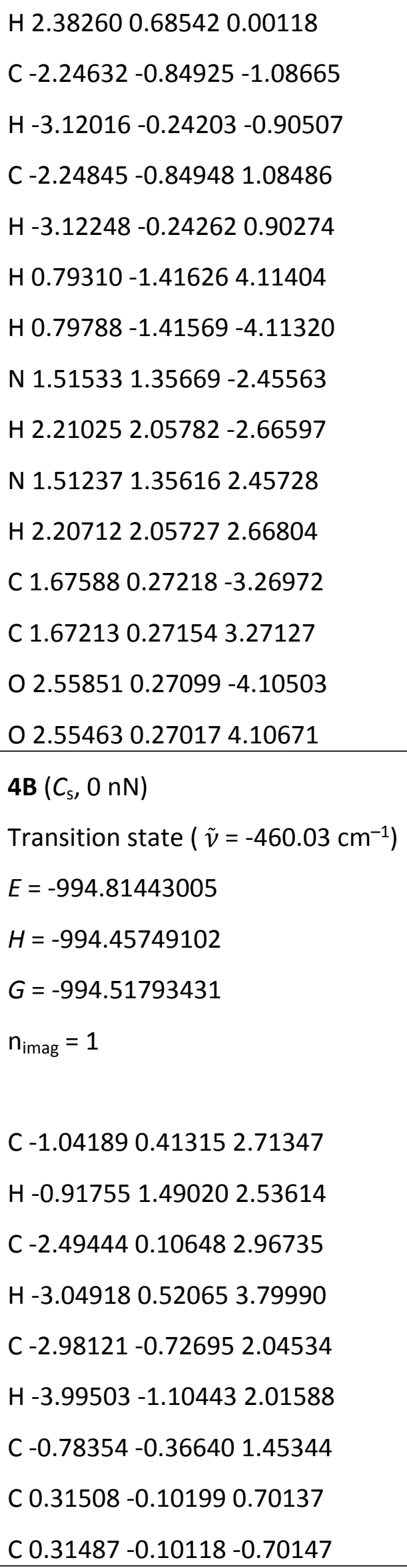




$$
\begin{array}{ll}
C-0.78396 & -0.36461-1.45352 \\
\text { C }-1.04233 & 0.41553-2.71336 \\
\text { H }-0.91790 & 1.49260-2.53595 \\
\text { C }-2.49500 & 0.10936-2.96706 \\
\text { C }-2.98172 & -0.72438-2.04526
\end{array}
$$$$
\text { H -3.04976 0.52393 -3.79942 }
$$$$
\text { H -3.99560 -1.10167 -2.01591 }
$$$$
\text { C } 1.434780 .777701 .19198
$$$$
\text { H } 1.047571 .804441 .29209
$$$$
\text { C } 1.434570 .77895-1.19173
$$$$
\text { H } 1.047321 .80572 \text {-1.29148 }
$$$$
\text { C - } 0.08444-0.00771-3.83401
$$$$
\text { H -0.23996 -1.06782 -4.05475 }
$$$$
\text { C -0.08405 -0.01090 } 3.83385
$$$$
\text { H -0.23964 -1.07120 4.05380 }
$$$$
\text { C } 2.403640 .761430 .00008
$$$$
\text { H } 3.104061 .598130 .00021
$$$$
\text { H } 2.96432-0.17828-0.00010
$$$$
\text { C -1.92217 -1.13290 -1.08937 }
$$$$
\text { H -1.81182 -2.19185 -0.92792 }
$$$$
\text { C -1.92170 -1.13472 } 1.08921
$$$$
\text { H -1.81130 -2.19366 } 0.92730
$$$$
\text { H -0.311890.52659 4.75603 }
$$$$
\text { H -0.31226 } 0.53069-4.75570
$$$$
\text { N } 2.024310 .359882 .44894
$$$$
\text { H } 3.023540 .457532 .54204
$$$$
\text { N } 2.023990 .36239-2.44898
$$$$
\text { H } 3.023210 .46032-2.54207
$$$$
\text { C } 1.430970 .13861-3.66260
$$$$
\text { C } 1.431310 .135513 .66247
$$

O $2.12743-0.00515-4.64760$ 
O $2.12781-0.008544 .64740$

4B $\left(C_{\mathrm{s}}, 2 \mathrm{nN}\right)$

Transition state $\left(\tilde{v}=-345.25 \mathrm{~cm}^{-1}\right)$

$E=-994.79744385$

$H=-994.44157138$

$G=-994.50161079$

$\mathrm{n}_{\text {imag }}=1$

C -1.025940.16782 2.93282

H-1.03927 1.25930 3.05809

C -2.37076 -0.41402 3.30738

H -2.71336 -0.39049 4.37256

C -2.94366 -0.98971 2.23894

H -3.90118 -1.49539 2.24643

C -0.88607 -0.21256 1.47826

C 0.117870 .243940 .71440

C $0.117810 .24428-0.71435$

C $-0.88622-0.21183-1.47828$

C -1.02605 0.16885 -2.93280

H -1.03919 1.26036 -3.05808

C -2.37101-0.41265 -3.30740

C -2.94397-0.98842-2.23902

H -2.71359-0.38893 -4.37259

H -3.90155 -1.49394-2.24658

C 1.219601 .143281 .21786

H 0.790512 .134721 .42817

C $1.219581 .14373-1.21770$

H $0.790552 .13523-1.42783$

C $0.12200-0.40197-3.77013$

H 0.18057 -1.48337 -3.61066

C $0.12218-0.403073 .77008$ 


$$
\begin{aligned}
& \text { H } 0.18082 \text {-1.48448 } 3.61045 \\
& \text { C } 2.149141 .247470 .00009 \\
& \text { H } 2.757732 .153510 .00013 \\
& \text { H } 2.813020 .378070 .00009 \\
& \text { C -1.99016 -1.06598 -1.05188 } \\
& \text { H -1.71089-2.10283 -0.92007 } \\
& \text { C -1.98986-1.06687 1.05178 } \\
& \text { H -1.71047 -2.10369 } 0.91987 \\
& \text { H -0.07723 -0.25964 } 4.83325 \\
& \text { H -0.07742 -0.25822 -4.83327 } \\
& \text { N } 1.946230 .669402 .38436 \\
& \text { H } 2.904450 .978212 .45214 \\
& \text { N } 1.946140 .67022-2.38435 \\
& \text { H } 2.904340 .97911-2.45212 \\
& \text { C } 1.535150 .12689-3.56921 \\
& \text { C } 1.535290 .125923 .56918 \\
& \text { O } 2.336940 .00189-4.47449 \\
& \text { O } 2.337100 .000994 .47446 \\
& \text { 4'A }\left(C_{s}, 0 \mathrm{nN}\right)
\end{aligned}
$$$$
\text { Transition state }\left(\tilde{v}=-522.85 \mathrm{~cm}^{-1}\right)
$$$$
E=-994.81102561
$$$$
H=-994.45369719
$$$$
G=-994.51433869
$$$$
\mathrm{n}_{\text {imag }}=1
$$$$
\text { C }-0.70143-0.765272 .63164
$$$$
\text { H -0.01765 -1.57629 2.34959 }
$$$$
\text { C -2.05767-1.36458 } 2.87935
$$$$
\text { H -2.24983 -2.06918 } 3.67895
$$$$
\text { C -2.94216-0.94779 } 1.97379
$$$$
\text { H -3.98132 -1.24545 } 1.92424
$$ 


$$
\begin{aligned}
& \text { C }-0.957670 .124371 .43815 \\
& \text { C } 0.041030 .711300 .70652 \\
& \text { C } 0.040770 .71200-0.70633 \\
& \text { C }-0.958280 .12573-1.43797 \\
& \text { C }-0.70251-0.76342-2.63174 \\
& \text { H -0.01896 -1.57461 -2.34979 } \\
& \text { C -2.05905 -1.36213 -2.87945 } \\
& \text { C - } 2.94336-0.94508-1.97385 \\
& \text { H -2.25154 -2.06663 -3.67904 } \\
& \text { H -3.98264 -1.24233 -1.92417 } \\
& \text { C } 1.408001 .113801 .21869 \\
& \text { H } 1.354552 .189601 .44411 \\
& \text { C } 1.407751 .11459-1.21850 \\
& \text { H } 1.354472 .19044 \text {-1.44368 } \\
& \text { C } 2.312450 .903880 .00003 \\
& \text { H } 3.190291 .553940 .00000 \\
& \text { H 2.64545 -0.13782 -0.00006 } \\
& \text { C - } 2.322730 .04660-1.06384 \\
& \text { H -2.86461 } 0.96705-0.92483 \\
& \text { C -2.32210 0.04455 } 1.06399 \\
& \text { H -2.864380.964720.92498 } \\
& \text { H } 2.965650 .47962-2.43293 \\
& \text { H } 2.966390 .478272 .43270 \\
& \text { C - } 0.05617-0.00063-3.79080 \\
& \text { H -0.52309 0.98288 -3.90132 } \\
& \text { H -0.18087 -0.52863 -4.73561 } \\
& \text { C -0.05510 -0.00274 } 3.79076 \\
& \text { H -0.522120.98067 } 3.90129 \\
& \text { H -0.17954 -0.53100 4.73549 } \\
& \text { N } 1.959540 .41509-2.37254 \\
& \text { N } 1.960260 .413792 .37242
\end{aligned}
$$




$$
\begin{aligned}
& \text { C } 1.442310 .16308-3.62017 \\
& \text { C } 1.443270 .161363 .62005 \\
& \text { O } 2.19625-0.01275-4.55556 \\
& \text { O } 2.19724-0.014374 .55543 \\
& \hline \text { 4'A }\left(C_{s}, 2 \mathrm{nN}\right)
\end{aligned}
$$$$
\text { Transition state }\left(\tilde{v}=-357.42 \mathrm{~cm}^{-1}\right)
$$$$
E=-994.792673
$$$$
H=-994.43654815
$$$$
G=-994.49591936
$$$$
\mathrm{n}_{\text {imag }}=1
$$

C - 2.921400 .205512 .42908

H -2.52370 1.142502 .01662

C -2.56305 -0.89479 1.45780

H -1.50986 -1.26857 1.39426

C -3.65394 -1.28404 0.78174

H -3.66703 -2.035410.00218

C -4.43815 0.23740 2.37917

C -5.211931.04083 3.13525

C -6.55126 1.432802 .78145

C-7.17877 1.037531 .65653

C -8.32873 1.781721 .00244

H -7.95760 2.724130 .57766

C -8.70700 $0.89016-0.15713$

C -7.79655 -0.08355 -0.30363

H -9.63989 $1.09231-0.74178$

H -7.81256 -0.83801-1.08015

C -4.78455 1.829054 .36057

H -5.04964 1.21380 5.23476

C -7.02461 2.48592 3.76726

H -7.54501 1.946704 .57435 


$$
\begin{aligned}
& \text { C -5.71743 } 3.039374 .33355 \\
& \text { H -5.83943 } 3.507765 .31273 \\
& \text { H -5.31493 } 3.776983 .63217 \\
& \text { C -6.85952 -0.167210.89568 } \\
& \text { H -7.12576 -1.06575 } 1.43576 \\
& \text { C -4.93127 -0.72800 1.40051 } \\
& \text { H -5.43371 -1.56032 } 1.87457 \\
& \text { H -7.75141 } 4.448603 .73512 \\
& \text { H -3.24470 } 3.124954 .93496 \\
& \text { C -9.438842.14973 } 1.99449 \\
& \text { H -9.58790 1.341332.71706 } \\
& \text { H -10.38542 2.31968 } 1.48298 \\
& \text { C -2.376850.08415 } 3.85759 \\
& \text { H -3.00697 -0.58621 } 4.45041 \\
& \text { H -1.36444 -0.31775 } 3.86188 \\
& \text { N -7.87319 } 3.557203 .27877 \\
& \text { N -3.39820 2.244874.46632 } \\
& \text { C -9.11363 } 3.447792 .70722 \\
& \text { C -2.28518 } 1.447214 .51500 \\
& \text { O -9.875564.391762.69972 } \\
& \text { O -1.24780 1.86322 4.98671 }
\end{aligned}
$$$$
\text { 4'B }^{\prime}\left(C_{\mathrm{s}}, 0 \mathrm{nN}\right)
$$$$
\text { Transition state }\left(\tilde{v}=-435.02 \mathrm{~cm}^{-1}\right)
$$$$
E=-994.80381555
$$$$
H=-994.44705017
$$$$
G=-994.50677855
$$$$
\mathrm{n}_{\text {imag }}=1
$$$$
\text { C -0.90601 } 0.138232 .93272
$$$$
\text { H -0.85121 -0.74274 } 3.59473
$$$$
\text { C }-0.89423-0.442461 .52688
$$ 


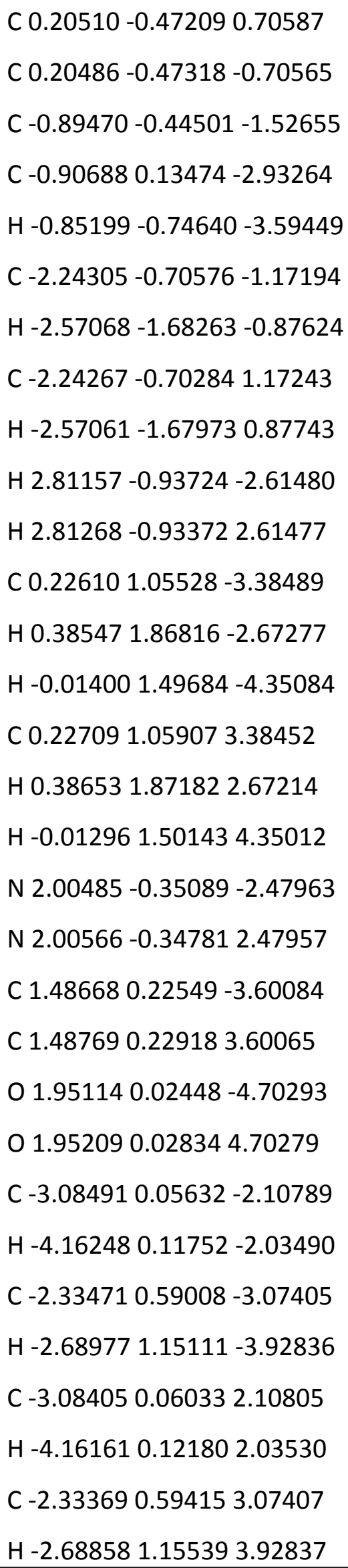




$$
\begin{aligned}
& \text { C } 2.48199-0.41417-0.00001 \\
& \text { H } 3.449710 .08955-0.00012 \\
& \text { H } 2.62789-1.499310 .00014 \\
& \text { C } 1.580780 .013341 .15270 \\
& \text { H } 1.558841 .110721 .10524 \\
& \text { C } 1.580390 .01175-1.15294 \\
& \text { H } 1.558411 .10915-1.10601
\end{aligned}
$$$$
\text { 4'B }\left(C_{\mathrm{s}}, 2 \mathrm{nN}\right)
$$$$
\text { Transition state }\left(\tilde{v}=-394.35 \mathrm{~cm}^{-1}\right)
$$$$
E=-994.78803036
$$$$
H=-994.4326943
$$$$
G=-994.4919411
$$$$
\mathrm{n}_{\text {imag }}=1
$$

C -2.848890.16476 3.23449

H -2.082720.89306 3.54599

C -3.895340.973362.47902

C -4.98283 1.540233 .03975

C -6.080972.166332.35526

C -6.23226 2.30512 1.02249

C -7.49508 2.812910.33896

H -7.36509 3.903880 .25330

C -5.37895 1.393144.50508

H -5.92540 0.44332 4.59875

C -7.17866 2.42042 3.38318

H -7.78870 1.507013 .43805

C -6.402462.50676 4.69203

H -7.03044 2.37060 5.57419

H -5.89055 3.473244 .75588

C -5.28270 $1.90661-0.01105$

H -4.47728 2.59607-0.21337 


$$
\begin{aligned}
& \text { C -3.567240.92975 } 1.05801 \\
& \text { H -3.09726 } 1.810320 .64734 \\
& \text { H -8.17185 4.22229 } 3.89353 \\
& \text { H -4.38637 } 2.060156 .25537 \\
& \text { C -8.831102.59059 } 1.05174 \\
& \text { H -8.90101 } 1.564891 .42455 \\
& \text { H -9.66194 } 2.756480 .36770 \\
& \text { C -3.29196-0.56758 4.50325 } \\
& \text { H -4.23368 -1.09634 4.33147 } \\
& \text { H -2.54467 -1.30101 4.80225 } \\
& \text { N -8.05135 } 3.547583 .15563 \\
& \text { N -4.31811 } 1.416385 .48389 \\
& \text { C -8.99399 } 3.603252 .17140 \\
& \text { C -3.406630.41607 } 5.65429 \\
& \text { O -9.853084.459362.15790 } \\
& \text { O -2.683870.37000 6.62722 } \\
& \text { C -6.15074 } 1.74190-1.24179 \\
& \text { H -5.76075 } 1.36862-2.18025 \\
& \text { C -7.36632 2.28267 -1.06907 } \\
& \text { H -8.16589 2.50911 -1.81766 } \\
& \text { C -2.63545 }-0.259860 .94825 \\
& \text { H -2.26567 -0.62056-0.00321 } \\
& \text { C - }-2.19821-0.661762 .15126 \\
& \text { H -1.36924 -1.36363 2.41794 } \\
& \text { 5A }\left(C_{\mathrm{s}}, 0 \mathrm{nN}\right)
\end{aligned}
$$$$
\text { Transition state }\left(\tilde{v}=-505.61 \mathrm{~cm}^{-1}\right)
$$$$
E=-814.65364518
$$$$
H=-814.23756774
$$$$
G=-814.2945462
$$$$
\mathrm{n}_{\text {imag }}=1
$$ 


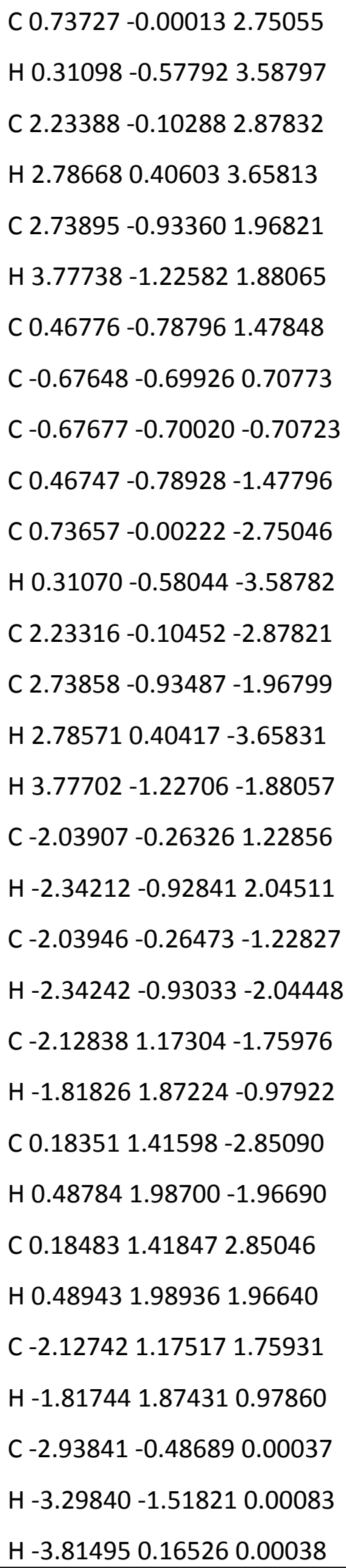




$$
\begin{aligned}
& \text { C } 1.65986-1.45219-1.10740 \\
& \text { H } 1.64211-2.50780-0.90644 \\
& \text { C } 1.65992-1.451421 .10815 \\
& \text { H } 1.64201-2.507000 .90735 \\
& \text { H -3.18370 } 1.385591 .95646 \\
& \text { H } 0.655481 .903423 .71139 \\
& \text { H -3.18472 } 1.38323-1.95690 \\
& \text { H } 0.654011 .90067-3.71203 \\
& \text { C -1.33125 } 1.48139-3.02051 \\
& \text { H -1.60457 } 2.48637-3.35513 \\
& \text { H -1.63297 } 0.80120-3.82678 \\
& \text { C -1.32999 } 1.484313 .01977 \\
& \text { H -1.60279 } 2.489623 .35387 \\
& \text { H -1.63218 } 0.804643 .82625
\end{aligned}
$$$$
5 \mathrm{~A}\left(C_{\mathrm{s}}, 2 \mathrm{nN}\right)
$$$$
\text { Transition state }\left(\tilde{v}=-372.54 \mathrm{~cm}^{-1}\right)
$$$$
E=-814.63401706
$$$$
H=-814.21917778
$$$$
G=-814.27543649
$$$$
\mathrm{n}_{\text {imag }}=1
$$

C 0.630210 .000332 .95872

H $0.32619-0.879623 .54638$

C 2.052780 .366843 .31198

H 2.315620 .606434 .37321

C 2.843870 .270042 .23435

H 3.909180 .466182 .22326

C $0.72831-0.414001 .49548$

C -0.33357 -0.72748 0.71972

C -0.33384-0.72753 -0.71983

C $0.72775-0.41374-1.49581$ 


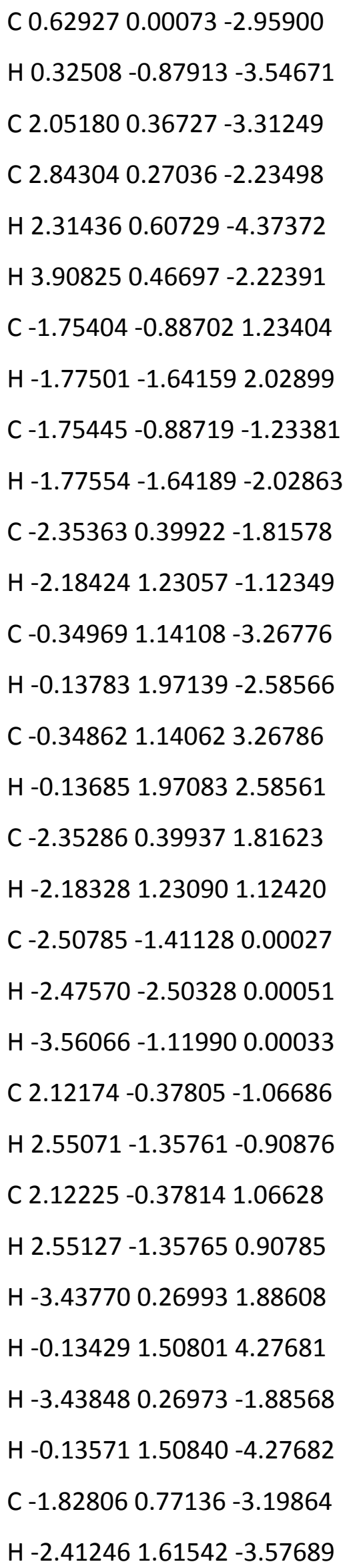




$$
\begin{aligned}
& \text { H -2.01960 -0.06141 -3.88695 } \\
& \text { C -1.82706 0.77108 } 3.19913 \\
& \text { H -2.41119 } 1.615273 .57747 \\
& \text { H - } 2.01869-0.061713 .88742 \\
& \text { 5B }\left(C_{s}, 0 \mathrm{nN}\right) \\
& \text { Transition state }\left(\tilde{v}=-411.11 \mathrm{~cm}^{-1}\right) \\
& E=-814.65423429 \\
& H=-814.23886927 \\
& G=-814.29597855 \\
& n_{\text {imag }}=1 \\
& \text { C -3.43278 -0.58755 } 2.97454 \\
& \text { H -3.97075 -1.173413.73399 } \\
& \text { C -3.08362 -1.47094 } 1.81018 \\
& \text { H -2.42348 -2.32553 } 1.89962 \\
& \text { C -3.64439 -1.03020 0.68460 } \\
& \text { H -3.54860 -1.49075 -0.29028 } \\
& \text { C - } 4.356040 .432722 .33660 \\
& \text { C -5.13930 1.28858 } 3.07412 \\
& \text { C -6.43741 1.70594 2.71137 } \\
& \text { C -7.09605 } 1.314371 .56996 \\
& \text { C -8.59223 } 1.071751 .52880 \\
& \text { H -8.90020 0.40989 2.35124 } \\
& \text { C -8.761930.35949 } 0.21648 \\
& \text { C -7.60038 0.25034 -0.42726 } \\
& \text { H -9.72586 0.02888 -0.15148 } \\
& \text { H -7.45453 -0.22102 -1.39064 } \\
& \text { C -4.91483 } 1.560934 .55002 \\
& \text { H -5.17159 0.65397 5.11917 } \\
& \text { C -7.14304 2.27732 } 3.92674 \\
& \text { H - 7.72826 1.47634 4.40460 }
\end{aligned}
$$




$$
\begin{aligned}
& \text { C -8.10483 } 3.426683 .63413 \\
& \text { H -7.60752 } 4.156742 .98550 \\
& \text { C -9.395982.37742 1.62961 } \\
& \text { H -8.97503 } 3.094940 .91779 \\
& \text { C -2.212820.06645 } 3.64159
\end{aligned}
$$$$
\text { H -1.774930.77788 2.93399 }
$$$$
\text { C -3.484761.941314.92705 }
$$$$
\text { H -3.12439 2.714964.23960 }
$$$$
\text { C -5.975982.62164 } 4.85650
$$$$
\text { H -6.271312.61732 } 5.90752
$$$$
\text { H -5.58957 } 3.622064 .62578
$$$$
\text { C -6.525120.922560.33497 }
$$$$
\text { H -5.95398 } 1.65377-0.21399
$$$$
\text { C - } 4.382240 .227810 .93556
$$$$
\text { H -4.11764 } 1.055550 .29761
$$$$
\text { H -3.49921 2.39446 5.92402 }
$$$$
\text { H -1.45508 -0.70380 } 3.81836
$$$$
\text { H -8.32132 } 3.943924 .57489
$$$$
\text { H -10.42336 2.18290 } 1.30496
$$$$
\text { C -9.44070 2.98979 } 3.02814
$$$$
\text { H -10.11228 } 3.852813 .00050
$$$$
\text { H -9.90605 2.26775 } 3.71101
$$$$
\text { C - } 2.510660 .761724 .96853
$$$$
\text { H -1.56264 } 1.104665 .39319
$$$$
\text { H -2.90425 } 0.017415 .67231
$$$$
\text { 5B }\left(C_{\mathrm{s}}, 2 \mathrm{nN}\right)
$$$$
\text { Transition state }\left(\tilde{v}=-368.71 \mathrm{~cm}^{-1}\right)
$$$$
E=-814.63844888
$$$$
H=-814.22372987
$$$$
G=-814.28034605
$$

$\mathrm{n}_{\text {imag }}=1$ 
C 0.832580 .000692 .94602

H 1.13735 -1.05321 3.02926

C 2.001220 .891103 .29303

H 2.358810 .946624 .35265

C 2.387471 .597852 .22153

H 3.189982 .325392 .21111

C 0.544870 .351901 .49223

C $-0.35275-0.299710 .71924$

C -0.35250 -0.30018 -0.71920

C $0.545610 .35077-1.49209$

C $0.83380-0.00119-2.94564$

H 1.13844 -1.05518 -3.02864

C $2.002710 .88878-3.29265$

C $2.389161 .59549-2.22119$

H 2.360540 .94385 -4.35222

H $3.192142 .32251-2.21071$

C -1.32066 -1.35241 1.22939

H -0.74962 -2.25365 1.49697

C -1.32018 -1.35331 -1.22931

H -0.74905 -2.25464 -1.49646

C -2.16468 -0.94190 -2.43533

H -2.62519 0.02920 -2.21871

C -0.36225 0.20172 -3.88432

H -0.79705 $1.19014-3.70047$

C -0.364010.20404 3.88420

H -0.79877 1.19245 3.69994

C -2.16584-0.94011 2.43476

H -2.62655 0.030882 .21788

C -2.18567-1.64802 0.00002

H -2.59191 -2.661340.00017 


$$
\begin{aligned}
& \text { H -3.03832 -0.95824 0.00003 } \\
& \text { C } 1.422531 .43365-1.06092 \\
& \text { H } 0.913932 .37563-0.91060 \\
& \text { C } 1.421261 .435191 .06112 \\
& \text { H } 0.912222 .376970 .91079 \\
& \text { H -2.98900 -1.65668 2.52879 } \\
& \text { H } 0.009480 .214814 .91356 \\
& \text { H -2.98783 -1.65843 -2.52955 } \\
& \text { H } 0.011840 .21202-4.91349 \\
& \text { C - } 1.43605-0.87570-3.77358 \\
& \text { H -2.17548 -0.71191 -4.56308 } \\
& \text { H -0.98098 }-1.85167-3.98272 \\
& \text { C - } 1.43787-0.873323 .77325 \\
& \text { H - } 2.17758-0.709334 .56248 \\
& \text { H - } 0.98301-1.849303 .98250
\end{aligned}
$$$$
\text { 5'A }\left(C_{s}, 0 \mathrm{nN}\right)
$$$$
\text { Transition state }\left(\tilde{v}=-528.61 \mathrm{~cm}^{-1}\right)
$$$$
E=-814.64629106
$$$$
H=-814.2298495
$$$$
G=-814.2874444
$$$$
\mathrm{n}_{\text {imag }}=1
$$

\section{C -3.17649 0.41492 2.24374}

H -3.14058 1.437201 .84942

C -3.01980 -0.55252 1.10360

H -2.12052 -0.61004 0.50264

C -4.09974 -1.322230.97186

H -4.22717 -2.11228 0.24288

C - 4.586200 .119652 .72681

C -5.32740 1.006713 .48256

C -6.64267 1.390003 .13439 


$$
\begin{aligned}
& \text { C -7.28619 0.90720 } 2.01189 \\
& \text { C -7.94799 } 1.807350 .98302 \\
& \text { H -7.280612.647620.75912 } \\
& \text { C -8.02657 } 0.91196-0.22204 \\
& \text { C -7.56438 -0.30766 } 0.05181 \\
& \text { H -8.43735 } 1.23876 \text {-1.16947 } \\
& \text { H -7.53502 -1.14161 -0.63774 } \\
& \text { C -4.76767 1.99486 } 4.49404 \\
& \text { H -4.98749 } 1.563065 .48295 \\
& \text { C-7.017232.65039 } 3.89848 \\
& \text { H -7.52770 2.30345 } 4.81047 \\
& \text { C -5.65973 } 3.224314 .30978 \\
& \text { H -5.71132 } 3.856195 .19988
\end{aligned}
$$$$
\text { H -5.26482 } 3.834383 .48804
$$$$
\text { C -7.10851-0.38019 1.45564 }
$$$$
\text { H -7.37345 -1.25020 } 2.03242
$$$$
\text { C }-5.11250-0.963171 .98612
$$$$
\text { H -5.62417 -1.75970 2.49949 }
$$$$
\text { C -9.305472.38162 } 1.40986
$$$$
\text { H -10.05199 } 1.583151 .38795
$$$$
\text { H -9.61188 } 3.113520 .65576
$$$$
\text { C -2.08580 0.28345 } 3.31549
$$$$
\text { H -1.92099 -0.77631 } 3.52786
$$$$
\text { H -1.151320.66420 } 2.89122
$$$$
\text { C -7.96360 } 3.648703 .23140
$$$$
\text { H -8.152994.445873.95647 }
$$$$
\text { H -7.46862 } 4.132742 .38296
$$$$
\text { C -9.29989 } 3.034002 .80156
$$$$
\text { H -10.08094 } 3.797842 .82865
$$$$
\text { H -9.58455 2.28744 } 3.54948
$$$$
\text { C -3.26564 2.27840 } 4.47501
$$ 


$$
\begin{aligned}
& \text { H -2.99450 2.82721 } 3.56701 \\
& \text { H -3.05578 2.95931 } 5.30525 \\
& \text { C -2.40398 } 1.020044 .62593 \\
& \text { H -2.92068 0.33562 5.30591 } \\
& \text { H -1.46163 1.27542 5.11681 } \\
& \text { 5'A }\left(C_{s}, 2 \mathrm{nN}\right) \\
& \text { Transition state }\left(\tilde{v}=-367.20 \mathrm{~cm}^{-1}\right) \\
& E=-814.62270188 \\
& H=-814.20755461 \\
& G=-814.26427983 \\
& \mathrm{n}_{\text {imag }}=1 \\
& \text { C -2.98927 -0.08224 2.82066 } \\
& \text { H -2.467550.61442 2.15129 } \\
& \text { C - } 2.80194-1.472212 .26253 \\
& \text { H -1.79778 -1.964912.32196 } \\
& \text { C -3.96307 -1.94687 } 1.79018 \\
& \text { H -4.09872 -2.91828 1.33051 } \\
& \text { C -4.49939 0.13038 2.74190 } \\
& \text { C -5.15711 1.23805 } 3.15746 \\
& \text { C -6.47827 } 1.616302 .71166 \\
& \text { C -7.21450 0.91311 } 1.81905 \\
& \text { C -8.36810 } 1.477120 .99231 \\
& \text { H -8.00108 2.23358 0.28663 } \\
& \text { C -8.83284 } 0.291020 .18300 \\
& \text { C -8.01741 -0.75515 } 0.37602 \\
& \text { H -9.76674 0.36869 -0.43056 } \\
& \text { H -8.12573 -1.72535 -0.09333 } \\
& \text { C -4.56768 2.37395 } 3.98131 \\
& \text { H -4.78590 } 2.150145 .03666 \\
& \text { C -6.79627 3.00942 } 3.23616
\end{aligned}
$$




$$
\begin{aligned}
& \text { H -7.34510 } 2.875884 .18090 \\
& \text { C -5.41485 } 3.576983 .56485 \\
& \text { H -5.44655 4.35906 4.32708 } \\
& \text { H -4.98562 } 4.016622 .65584 \\
& \text { C -7.04976-0.50684 } 1.52282 \\
& \text { H -7.35756 -1.14170 2.34202 } \\
& \text { C }-5.13348-1.061862 .18901 \\
& \text { H -5.70886 -1.60463 2.92602 } \\
& \text { C -9.43660 2.13627 } 1.87263 \\
& \text { H -9.54921 } 1.539092 .78179 \\
& \text { H -10.40420 } 2.103661 .36330 \\
& \text { C -2.449720.13812 } 4.23875 \\
& \text { H -3.17603 -0.26495 4.95010 } \\
& \text { H -1.53219 -0.44142 4.37694 } \\
& \text { C -7.61630 3.925912.33462 } \\
& \text { H -7.15530 } 3.937451 .34171 \\
& \text { H -7.52381 } 4.949082 .71308 \\
& \text { C -9.10555 } 3.595072 .22412 \\
& \text { H -9.53585 } 4.251951 .46189 \\
& \text { H -9.60308 } 3.856493 .16299 \\
& \text { C -3.06971 2.62574 } 3.84655 \\
& \text { H -2.85110 } 3.617844 .25473 \\
& \text { H -2.82396 } 2.685332 .78143 \\
& \text { C -2.15859 } 1.614934 .54520 \\
& \text { H -2.21128 } 1.770365 .62716 \\
& \text { H -1.12746 } 1.842194 .25770
\end{aligned}
$$$$
\mathbf{5}^{\prime} \mathrm{B}\left(C_{\mathrm{s}}, 0 \mathrm{nN}\right)
$$$$
\text { Transition state }\left(\tilde{v}=-441.42 \mathrm{~cm}^{-1}\right)
$$$$
E=-814.63339555
$$$$
H=-814.21767731
$$$$
G=-814.27456349
$$ 


$$
\begin{aligned}
& \mathrm{n}_{\text {imag }}=1 \\
& \text { C } 0.71851-0.001322 .91158 \\
& \text { H } 0.382620 .782783 .61268 \\
& \text { C } 2.21727-0.001253 .05712 \\
& \text { H } 2.72407-0.450583 .90168 \\
& \text { C } 2.779890 .737952 .09958 \\
& \text { H } 3.829770 .990012 .02647 \\
& \text { C } 0.533870 .594321 .52169 \\
& \text { C - } 0.547350 .347730 .70703 \\
& \text { C -0.54733 0.34804 -0.70690 } \\
& \text { C } 0.534000 .59513-1.52130 \\
& \text { C } 0.718480 .00050-2.91152 \\
& \text { H } 0.382450 .78507-3.61207 \\
& \text { C } 2.217210 .00054-3.05710 \\
& \text { C } 2.779900 .73949-2.09940 \\
& \text { H } 2.72396-0.44844-3.90187 \\
& \text { H } 3.829720 .99186 \text {-2.02641 } \\
& \text { C -1.82606 -0.34290 } 1.16658 \\
& \text { H -1.65156 -1.42833 } 1.10438 \\
& \text { C }-1.82625-0.34222-1.16662 \\
& \text { H -1.65217 -1.42772 -1.10463 } \\
& \text { C -2.76987-0.043810.00009 } \\
& \text { H -3.66297 -0.673370.00030 } \\
& \text { H -3.07751 1.007810.00006 } \\
& \text { C } 1.752031 .22802-1.16635 \\
& \text { H } 1.782982 .26006-0.87751 \\
& \text { C } 1.752011 .227041 .16678 \\
& \text { H } 1.783192 .259030 .87799 \\
& \text { C - } 0.05528-1.26277-3.26357 \\
& \text { H } 0.03245 \text {-1.98080 -2.44394 }
\end{aligned}
$$




$$
\begin{aligned}
& \text { H } 0.40628-1.73899-4.13322 \\
& \text { C - } 0.05511-1.264953 .26320 \\
& \text { H } 0.03292-1.983202 .44375 \\
& \text { H } 0.40631 \text { - } 1.741084 .13297 \\
& \text { C - } 2.24548-0.04569-2.59728 \\
& \text { H -2.03227 } 1.00172-2.83103 \\
& \text { H -3.32603 -0.17222 -2.70898 } \\
& \text { C -1.52996 -0.97939-3.58926 } \\
& \text { H -1.59731 -0.55265 -4.59487 } \\
& \text { H -2.06404 -1.93391 -3.62652 } \\
& \text { C -2.24534-0.04730 } 2.59729 \\
& \text { H -3.32591 -0.17379 2.70893 } \\
& \text { H -2.031910.99998 } 2.83129 \\
& \text { C - } 1.52990-0.981663 .58879 \\
& \text { H -2.06393 -1.93626 3.62551 } \\
& \text { H - } 1.59738-0.555444 .59459
\end{aligned}
$$$$
\mathbf{5}^{\prime} \mathrm{B}\left(C_{\mathrm{s}}, 2 \mathrm{nN}\right)
$$$$
\text { Transition state }\left(\tilde{v}=-390.88 \mathrm{~cm}^{-1}\right)
$$$$
E=-814.61725136
$$$$
H=-814.2028644
$$$$
G=-814.25927275
$$$$
\mathrm{n}_{\text {imag }}=1
$$

C 0.657420 .000313 .04105

H 0.475690 .987293 .49760

C $2.08769-0.347213 .37645$

H $2.36870-0.547434 .44106$

C $2.86487-0.203252 .29328$

H $3.94160-0.318742 .27559$

C 0.693630 .227491 .52951

C -0.391520 .222940 .72163$ 


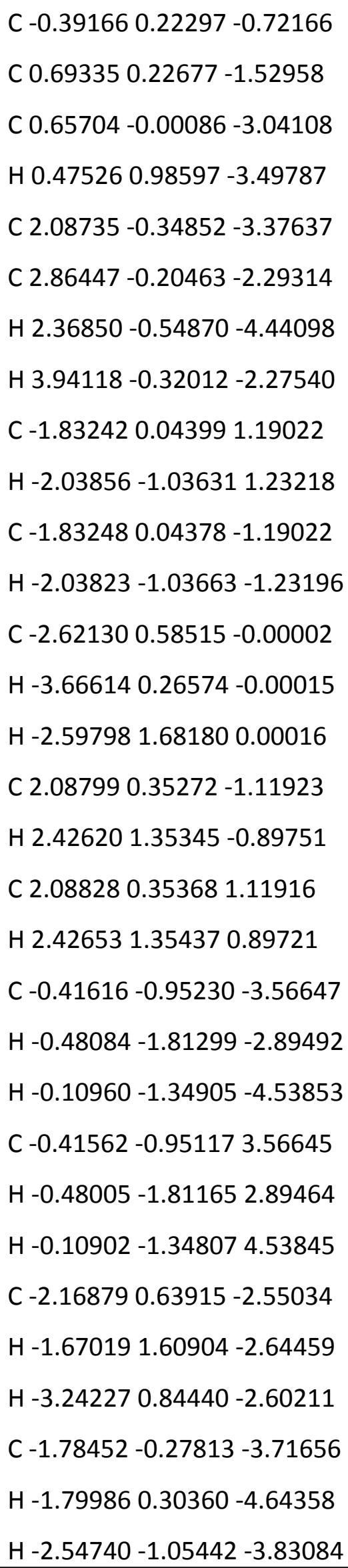




$$
\begin{aligned}
& \text { C -2.16842 0.63980 2.55029 } \\
& \text { H -3.24187 } 0.845072 .60212 \\
& \text { H -1.66982 } 1.609742 .64445 \\
& \text { C -1.78392 }-0.277083 .71667 \\
& \text { H - } 2.54689-1.053203 .83149 \\
& \text { H - } 1.798740 .305054 .64345
\end{aligned}
$$$$
1 \mathrm{~A}\left(C_{\mathrm{s}}, 0 \mathrm{nN}\right)
$$$$
E=-733.60401842
$$$$
H=-733.29401333
$$$$
G=-733.34483812
$$$$
\mathrm{n}_{\text {imag }}=0
$$

C $0.49450-0.005322 .78522$

H $0.80964-0.711073 .57019$

C 1.592711 .030682 .62014

H 1.766111 .819053 .34277

C 2.328690 .809481 .53753

H 3.195741 .393591 .25439

C $0.52595-0.634641 .42033$

C -0.57086 -0.89314 0.73030

C $-0.57083-0.89324-0.73032$

C $0.52594-0.63453-1.42032$

C $0.49445-0.00513-2.78517$

H $0.81028-0.71061-3.57010$

C $1.591951 .03155-2.61961$

C $2.327970 .81041-1.53700$

H $1.764921 .82028-3.34196$

H 3.194641 .39495 -1.25357

C -1.97106 -0.67599 1.23322

H -2.45289 -1.63584 1.46221

C $-1.97107-0.67650-1.23329$ 


$$
\begin{aligned}
& \text { H -2.45277 -1.63653 -1.46182 } \\
& \text { C -1.97615 0.18580 -2.46490 } \\
& \text { H -2.93702 } 0.56821 \text {-2.79769 } \\
& \text { C -0.87845 0.50171 -3.14167 } \\
& \text { H -0.95509 1.13687 -4.01956 } \\
& \text { C -0.878180.50239 } 3.14133 \\
& \text { H -0.95463 } 1.137824 .01904 \\
& \text { C -1.975920.186862.46445 } \\
& \text { H -2.936670.569832.79696 } \\
& \text { C } 1.88133-0.42940-0.78178 \\
& \text { H } 2.54673 \text {-1.24662 -1.08821 } \\
& \text { C } 1.88140-0.429770 .78180 \\
& \text { H } 2.54649 \text {-1.24741 } 1.08779 \\
& \text { C -2.67116 -0.05236 -0.00016 } \\
& \text { H -3.75128 -0.20920 -0.00012 } \\
& \text { H -2.48527 } 1.02572-0.00040
\end{aligned}
$$$$
1 \mathrm{~A}\left(C_{\mathrm{s}}, 2 \mathrm{nN}\right)
$$$$
E=-733.57752848
$$$$
H=-733.26938959
$$$$
G=-733.32008473
$$$$
\mathrm{n}_{\text {imag }}=1
$$

C $0.49162-0.000432 .87760$

H $0.94927-0.782683 .50454$

C 1.316311 .264543 .02145

H 1.405681 .729894 .03353

C 1.950531 .558061 .87537

H 2.632932 .390661 .74895

C $0.66058-0.316451 .42146$

C -0.29090 -0.90407 0.72289

C -0.29091 -0.90406 -0.72289 


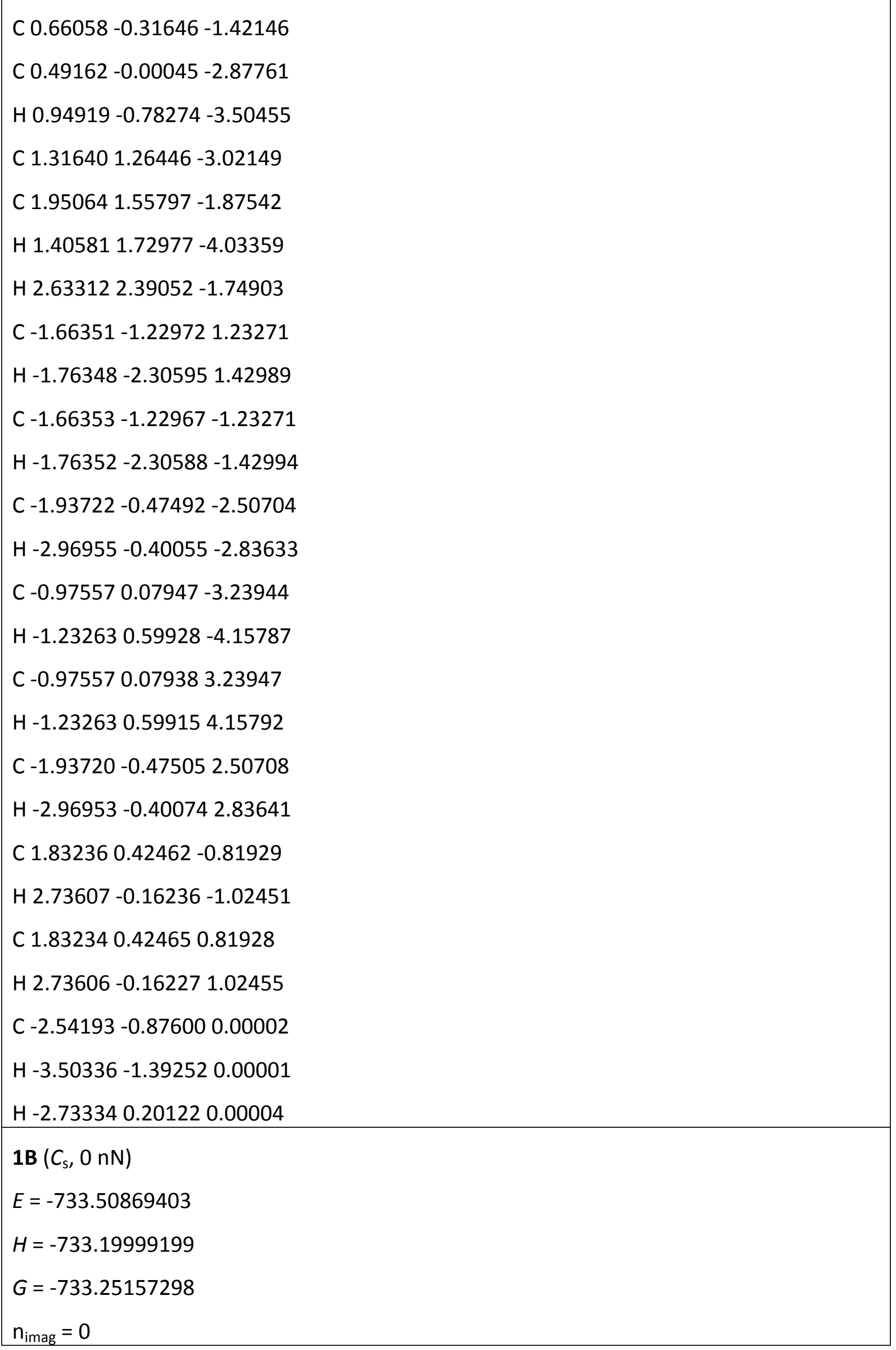


C $0.55926-0.000282 .86054$

H 1.029410 .899453 .28078

C 1.53023 -1.15555 3.09494

H 1.88631 -1.43644 4.07854

C $1.98650-1.682531 .95352$

H 2.73419 -2.46323 1.89165

C 0.551550 .055471 .37886

C -0.20710 0.894720.71333

C -0.20710 0.89472 -0.71333

C $0.551550 .05547-1.37886$

C $0.55926-0.00028-2.86054$

H $1.029410 .89945-3.28078$

C $1.53023-1.15555-3.09494$

C 1.98650 -1.68253 -1.95352

H 1.88631 -1.43644 -4.07854

H 2.73419 -2.46323 -1.89165

C -1.42766 1.54841 1.26456

H -1.29606 2.610261.50913

C -1.42766 $1.54841-1.26456$

H -1.29606 2.61026-1.50913

C - $1.794880 .79718-2.54250$

H -2.81751 $0.87951-2.89926$

C -0.91981 $0.08086-3.26230$

H -1.24863 -0.39707-4.17982

C -0.919810.08086 3.26230

H -1.24863 -0.39707 4.17982

C -1.794870.79718 2.54250

H -2.81751 0.879512 .89926

C $1.11085-1.21476-0.79561$

H 0.26486 -1.89398 -0.95672 
C $1.11085-1.214760 .79561$
H $0.26486-1.893980 .95672$
C -2.36752 1.433510 .00000
H -2.83169 0.443170 .00000
H -3.16355 2.179740 .00000

$1 \mathrm{~B}\left(C_{\mathrm{s}}, 2 \mathrm{nN}\right)$

$E=-733.49785593$

$H=-733.19095926$

$G=-733.24256379$

$\mathrm{n}_{\text {imag }}=0$

C 0.536660 .000212 .88986

H 1.060370 .899643 .24653

C $1.42749-1.186763 .24176$

H 1.66217 -1.41729 4.31163

C $1.91916-1.764742 .12733$

H $2.62869-2.583362 .12683$

C $0.54173-0.037161 .40023$

C -0.189720 .808380 .71041$

C $-0.189720 .80838-0.71041$

C $0.54173-0.03716-1.40023$

C $0.536660 .00021-2.88986$

H $1.060370 .89964-3.24653$

C $1.42749-1.18676-3.24176$

C $1.91916-1.76474-2.12733$

H 1.66217 -1.41729 -4.31163

H 2.62869 -2.58336 -2.12683

C -1.33484 1.59457 1.25303

H -1.074992.63998 1.46811

C -1.33484 1.59457-1.25303

H -1.07499 2.63998 -1.46811 


$$
\begin{aligned}
& \text { C - } 1.760750 .92816-2.55451 \\
& \text { H }-2.776461 .08885-2.90340 \\
& \text { C -0.92607 } 0.19123-3.29768 \\
& \text { H -1.27233 }-0.23232-4.23531 \\
& \text { C -0.92607 } 0.191233 .29768 \\
& \text { H -1.27233 }-0.232324 .23531 \\
& \text { C - } 1.760750 .928162 .55451 \\
& \text { H }-2.776461 .088852 .90340 \\
& \text { C } 1.13193-1.30845-0.85708 \\
& \text { H } 0.29750-2.00898-0.94231 \\
& \text { C } 1.13193-1.308450 .85708 \\
& \text { H } 0.29750-2.008980 .94231 \\
& \text { C -2.28780 } 1.566620 .00000 \\
& \text { H }-2.842450 .623770 .00000 \\
& \text { H }-3.009882 .384570 .00000
\end{aligned}
$$$$
\text { 1'A }\left(C_{\mathrm{s}}, 0 \mathrm{nN}\right)
$$$$
E=-733.52344464
$$$$
H=-733.21456462
$$$$
G=-733.26559549
$$$$
\mathrm{n}_{\text {imag }}=0
$$

C 0.723100 .001932 .29774

H 1.374470 .623191 .65316

C $1.64072-1.153282 .62774$

H 2.40451 -1.12861 3.39574

C $1.51583-2.111641 .70809$

H 2.14878 -2.98876 1.64990

C $-0.24155-0.626271 .33826$

C -1.154530.122540.73142

C -1.15443 0.12265 -0.73146

C - $0.24162-0.62638-1.33830$ 


$$
\begin{aligned}
& \text { C } 0.723230 .00147-2.29779 \\
& \text { H } 1.375100 .62218 \text {-1.65318 } \\
& \text { C } 1.64005-1.15426-2.62822 \\
& \text { C } 1.51494-2.11258-1.70857 \\
& \text { H } 2.40359 \text {-1.12999 -3.39649 } \\
& \text { H } 2.14740 \text {-2.99007 -1.65062 } \\
& \text { C -1.48553 1.51094 } 1.21938 \\
& \text { H -2.57723 } 1.609621 .29183 \\
& \text { C -1.48507 } 1.51119-1.21930 \\
& \text { H -2.57673 } 1.61001-1.29215 \\
& \text { C } 0.038291 .02694-3.15718 \\
& \text { C } 0.037601 .026773 .15742 \\
& \text { C -1.04350 2.378690.00021 } \\
& \text { H -1.486913.375930.00023 } \\
& \text { H } 0.044952 .490020 .00044 \\
& \text { C } 0.30519-1.91040-0.81245 \\
& \text { H -0.39802 -2.71267-1.04845 } \\
& \text { C } 0.30557-1.910180 .81248 \\
& \text { H -0.39720 -2.71268 } 1.04899 \\
& \text { H } 0.373731 .221094 .17027 \\
& \text { H } 0.374621 .22141 \text {-4.16994 } \\
& \text { C -0.91860 1.77132 -2.59904 } \\
& \text { H -1.37707 2.56644 -3.17858 } \\
& \text { C -0.91958 } 1.770862 .59938 \\
& \text { H -1.378512.56561 } 3.17905 \\
& \text { 1'A }\left(C_{\mathrm{s}}, 1.8 \mathrm{nN}\right) \\
& E=-733.49825821 \\
& H=-733.19079963 \\
& G=-733.24216319 \\
& \mathrm{n}_{\text {imag }}=0
\end{aligned}
$$




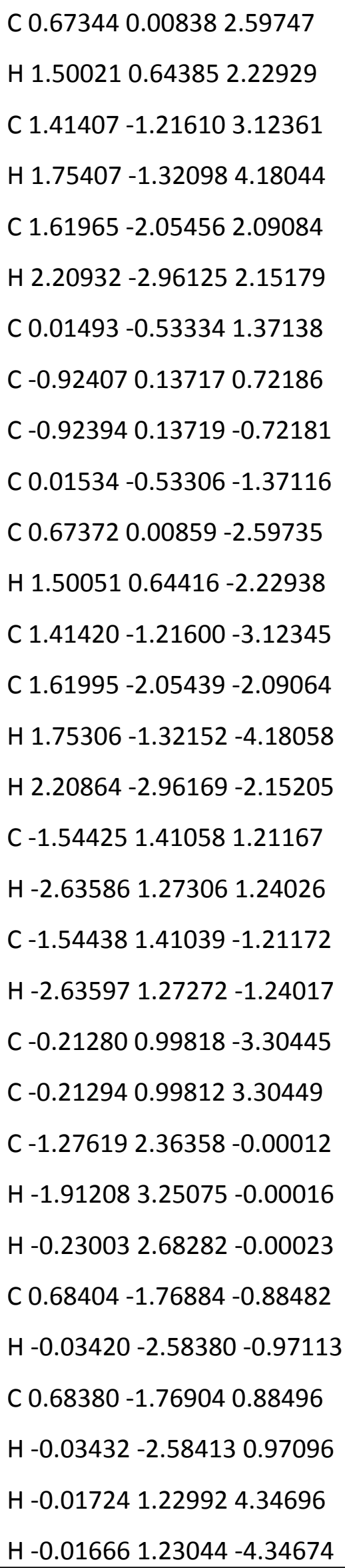




$$
\begin{aligned}
& C-1.117771 .71198-2.62873 \\
& H-1.659132 .49444-3.15238 \\
& C-1.117551 .712252 .62864 \\
& H-1.659212 .494423 .15243 \\
& \hline 1^{\prime} B\left(C_{s}, 0 n N\right) \\
& E=-733.5455086 \\
& H=-733.23604554 \\
& G=-733.28691687 \\
& \mathrm{n}_{\text {imag }}=0
\end{aligned}
$$

C $0.57946-0.000562 .86354$

H 1.316350 .536883 .48108

C $1.09671-1.437332 .72850$

H $0.96324-2.198173 .48802$

C $1.81460-1.593451 .61332$

H 2.34094 -2.50372 1.35063

C 0.710870 .365671 .42332

C -0.367460 .600360 .71421$

C -0.36746 0.60036 -0.71421

C $0.710870 .36567-1.42332$

C $0.57945-0.00056-2.86354$

H $1.316360 .53686-3.48108$

C 1.09669 -1.43734 -2.72849

C $1.81457-1.59346-1.61331$

H $0.96321-2.19818-3.48801$

H 2.34089 -2.50374 -1.35061

C -1.783550.61185 1.17626

H -2.12746 -0.42718 1.03508

C -1.78354 0.61186-1.17626

H - 2.12746 -0.42717 -1.03510

C $-0.789450 .37410-3.40743$ 


$$
\begin{aligned}
& \text { C -0.78945 } 0.374083 .40743 \\
& \text { C -2.49439 } 1.363730 .00000 \\
& \text { H -3.57789 } 1.237960 .00000 \\
& \text { H -2.25589 } 2.430300 .00000 \\
& \text { C } 1.91067-0.30480-0.78752 \\
& \text { H } 2.839610 .19188-1.09512 \\
& \text { C } 1.91067-0.304800 .78752 \\
& \text { H } 2.839610 .191901 .09511 \\
& \text { H -0.88603 } 0.401524 .48875 \\
& \text { H -0.88603 } 0.40154-4.48875 \\
& \text { C - }-1.824750 .78663-2.66763 \\
& \text { H -2.73733 } 1.11229-3.15639 \\
& \text { C - } 1.824760 .786602 .66763 \\
& \text { H - } 2.737341 .112263 .15639
\end{aligned}
$$$$
\text { 1'B }\left(C_{\mathrm{s}}, 2 \mathrm{nN}\right)
$$$$
E=-733.52036887
$$$$
H=-733.21270968
$$$$
G=-733.26377764
$$$$
\mathrm{n}_{\text {imag }}=0
$$

C $0.62550-0.000642 .87730$

H 1.498840 .584493 .21719

C $0.98926-1.468243 .13342$

H $0.97897-1.875964 .17475$

C $1.46772-2.027242 .00059$

H 1.87714 -3.02924 1.94442

C $0.59404-0.033891 .39633$

C -0.446740.36925 0.71366

C - $0.446660 .36937-0.71375$

C $0.59407-0.03393-1.39636$

C $0.62556-0.00082-2.87732$ 


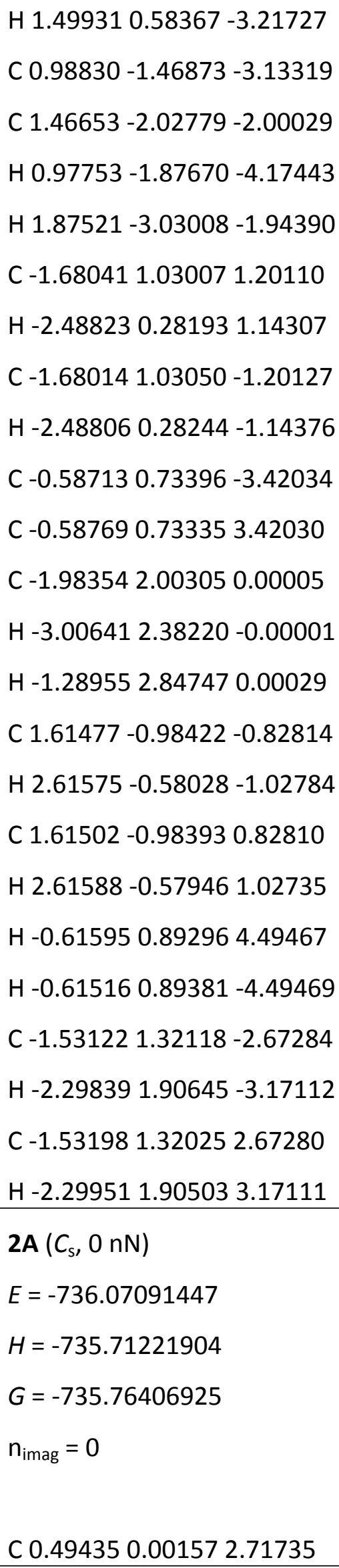




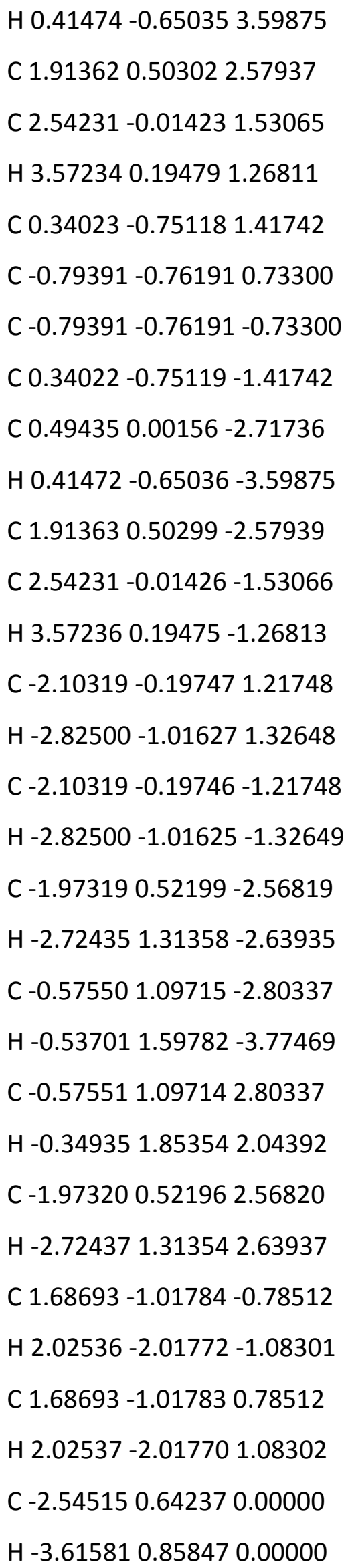




$$
\begin{aligned}
& \text { H -2.00633 1.597370.00001 } \\
& \text { H -2.19588 -0.18664 } 3.37206 \\
& \text { H -0.53704 } 1.597803 .77470 \\
& \text { H -2.19588 -0.18661 -3.37205 } \\
& \text { H -0.34932 } 1.85354-2.04391 \\
& \text { H } 2.355071 .196833 .28559 \\
& \text { H 2.35508 1.19679 -3.28562 } \\
& 2 \mathrm{~A}\left(C_{\mathrm{s}}, 2 \mathrm{nN}\right) \\
& E=-736.04299569 \\
& H=-735.68609579 \\
& G=-735.73828111 \\
& \mathrm{n}_{\text {imag }}=0 \\
& \text { C } 0.571170 .012072 .85185 \\
& \text { H } 0.96345-0.955303 .19194 \\
& \text { C } 1.645951 .070403 .08467 \\
& \text { H } 2.076191 .275674 .05776 \\
& \text { C } 2.077771 .630911 .94797 \\
& \text { H } 2.875442 .361441 .89072 \\
& \text { C } 0.483220 .064471 .37789 \\
& \text { C -0.29589 }-0.746490 .70944 \\
& \text { C }-0.29559-0.74683-0.70947 \\
& \text { C } 0.483680 .06401-1.37794 \\
& \text { C } 0.571610 .01179-2.85197 \\
& \text { H } 0.96414-0.95539 \text {-3.19232 } \\
& \text { C } 1.645901 .07067-3.08470 \\
& \text { C } 2.078391 .63031-1.94783 \\
& \text { H } 2.07531 \text { 1.27697 -4.05793 } \\
& \text { H } 2.875632 .36132 \text {-1.89063 } \\
& \text { C -1.37093 -1.60254 1.25943 } \\
& \text { H -1.02102 -2.62651 } 1.43355
\end{aligned}
$$




$$
\begin{aligned}
& \text { C - } 1.37072-1.60283-1.25944 \\
& \text { H -1.02105 -2.62688 -1.43364 } \\
& \text { C } 1.124571 .29545-0.80308 \\
& \text { H } 0.362422 .06575-0.97192 \\
& \text { C } 1.124271 .295780 .80299 \\
& \text { H } 0.362042 .066130 .97125 \\
& \text { C -2.32724 -1.63051-0.00006 } \\
& \text { H -3.00607 -2.48514-0.00001 } \\
& \text { H -2.93505 -0.71906 -0.00032 } \\
& \text { C -1.83402 -1.00241 -2.63253 } \\
& \text { H -1.96046 -1.82265 -3.34205 } \\
& \text { H - 2.82590 -0.55897 -2.51174 } \\
& \text { C - } 0.913040 .09386-3.30531 \\
& \text { H -1.28671 } 1.08624 \text {-3.03878 } \\
& \text { H - } 1.010220 .00094-4.39018 \\
& \text { C -1.83406 -1.00259 2.63284 } \\
& \text { H -2.82652 -0.56024 } 2.51299 \\
& \text { H -1.95898 -1.82316 3.34228 } \\
& \text { C - } 0.913440 .094213 .30531 \\
& \text { H -1.01042 0.001334.39021 } \\
& \text { H -1.28737 } 1.086473 .03873 \\
& \text { 2B }\left(C_{s}, 0 \mathrm{nN}\right) \\
& E=-735.98309582 \\
& H=-735.62558632 \\
& G=-735.68053754 \\
& \mathrm{n}_{\text {imag }}=0
\end{aligned}
$$$$
\text { C } 0.682590 .006612 .49013
$$$$
\text { H } 1.268620 .837232 .05781
$$$$
\text { C } 1.73719-1.043522 .76392
$$$$
\text { H } 2.43842-0.995413 .58904
$$ 


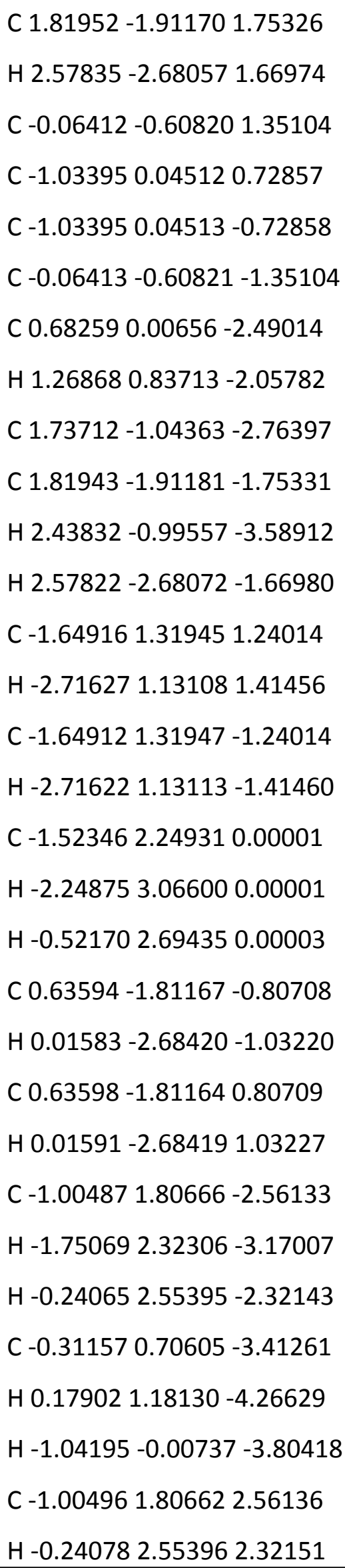




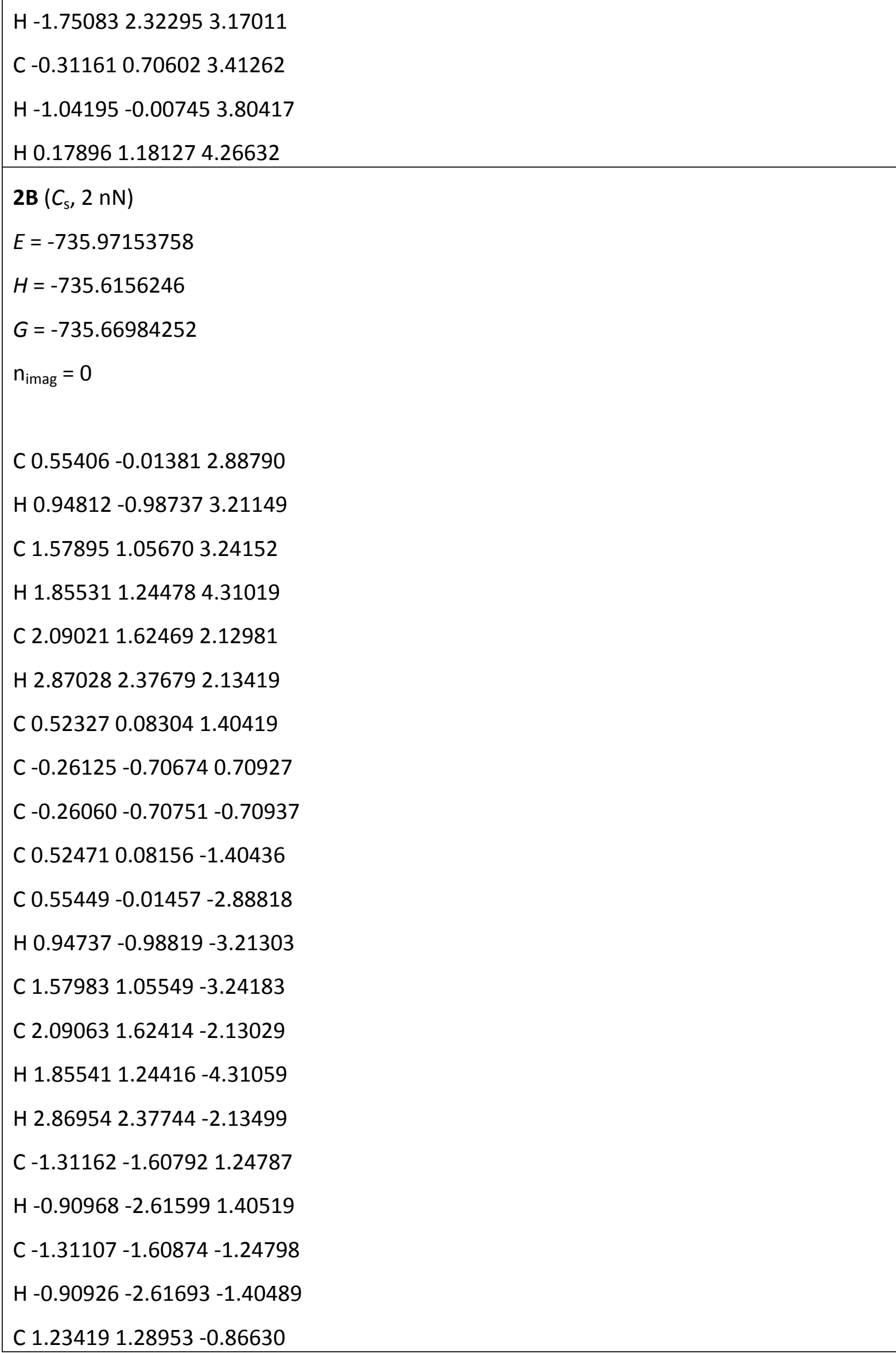




$$
\begin{aligned}
& \text { H } 0.497622 .09235-0.94993 \\
& \text { C } 1.233871 .290110 .86571 \\
& \text { H } 0.498022 .093680 .94847 \\
& \text { C - } 2.26622-1.66917-0.00022 \\
& \text { H -2.91515 -2.54676 -0.00006 } \\
& \text { H -2.90524 -0.77901-0.00070 } \\
& \text { C -1.81264 -1.06554-2.62417 } \\
& \text { H -1.90474 -1.90582 -3.31555 } \\
& \text { H - 2.82250 -0.66328 - 2.50813 } \\
& \text { C - } 0.935430 .04476-3.30878 \\
& \text { H -1.31803 } 1.03108 \text {-3.03188 } \\
& \text { H -1.04876 -0.04562 -4.39257 } \\
& \text { C -1.81351 -1.06490 2.62405 } \\
& \text { H -2.82285 -0.661392.50780 } \\
& \text { H -1.90703 -1.90570 3.31459 } \\
& \text { C - } 0.935450 .043753 .31041 \\
& \text { H -1.04722 -0.04977 } 4.39413 \\
& \text { H -1.31877 1.03073 3.03701 }
\end{aligned}
$$$$
\text { 2'A }\left(C_{\mathrm{s}}, 0 \mathrm{nN}\right)
$$$$
E=-736.01023307
$$$$
H=-735.65233185
$$$$
G=-735.70480799
$$$$
\mathrm{n}_{\text {imag }}=0
$$

C $0.62347-0.000622 .72654$

H 1.305610 .847762 .54769

C 1.54217 -1.12715 3.17503

H 1.87272 -1.23386 4.23954

C $1.90573-1.854762 .10126$

H $2.63011-2.660272 .12917$

C $0.16279-0.487571 .38769$ 


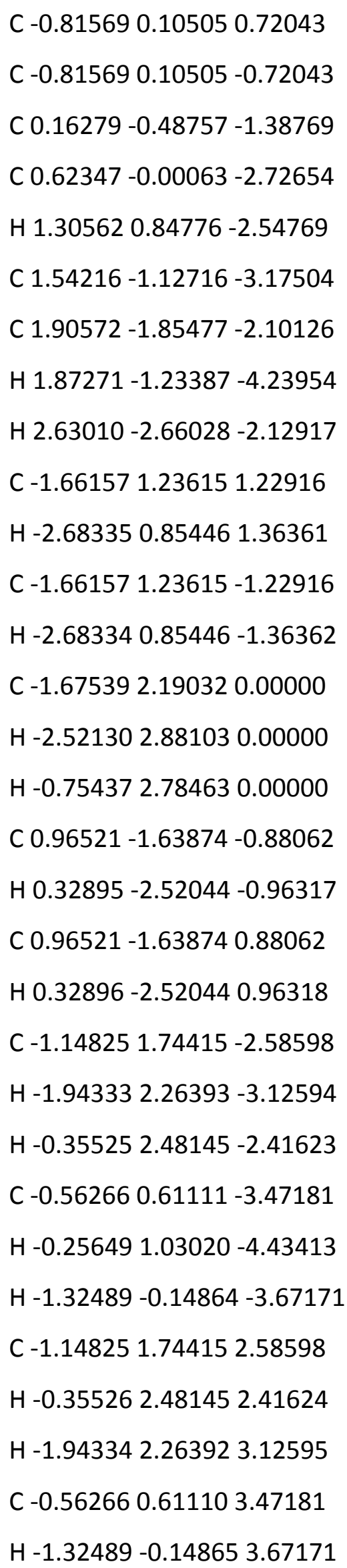




$$
\begin{aligned}
& \text { H -0.25650 1.030194.43414 } \\
& \text { 2'A }\left(C_{s}, 2 \mathrm{nN}\right) \\
& E=735.98709275 \\
& H=735.63101058 \\
& G=735.68353196 \\
& \mathrm{n}_{\text {imag }}=0
\end{aligned}
$$

C $0.62347-0.000622 .72654$

H 1.305610 .847762 .54769

C $1.54217-1.127153 .17503$

H 1.87272 -1.23386 4.23954

C $1.90573-1.854762 .10126$

H 2.63011 -2.66027 2.12917

C $0.16279-0.487571 .38769$

C - 0.815690 .105050 .72043

C -0.81569 0.10505 -0.72043

C $0.16279-0.48757-1.38769$

C $0.62347-0.00063-2.72654$

H $1.305620 .84776-2.54769$

C $1.54216-1.12716-3.17504$

C $1.90572-1.85477-2.10126$

H $1.87271-1.23387-4.23954$

H 2.63010 -2.66028 -2.12917

C -1.66157 1.23615 1.22916

H -2.683350.854461.36361

C -1.66157 1.23615 -1.22916

H -2.68334 $0.85446-1.36362$

C -1.67539 2.190320.00000

H - 2.521302 .881030 .00000

H -0.75437 2.78463 0.00000 


$$
\begin{aligned}
& \text { C } 0.96521-1.63874-0.88062 \\
& \text { H } 0.32895-2.52044-0.96317 \\
& \text { C } 0.96521-1.638740 .88062 \\
& \text { H } 0.32896-2.520440 .96318 \\
& \text { C -1.14825 } 1.74415-2.58598 \\
& \text { H -1.94333 2.26393 -3.12594 } \\
& \text { H -0.35525 } 2.48145-2.41623 \\
& \text { C - } 0.562660 .61111-3.47181 \\
& \text { H -0.25649 } 1.03020-4.43413 \\
& \text { H -1.32489 -0.14864 -3.67171 } \\
& \text { C -1.14825 } 1.744152 .58598 \\
& \text { H -0.35526 2.48145 2.41624 } \\
& \text { H -1.94334 2.26392 } 3.12595 \\
& \text { C - } 0.562660 .611103 .47181 \\
& \text { H -1.32489 -0.14865 } 3.67171 \\
& \text { H -0.25650 1.03019 4.43414 }
\end{aligned}
$$$$
\text { 2'B }\left(C_{\mathrm{s}}, 0 \mathrm{nN}\right)
$$$$
E=-736.03961073
$$$$
H=-735.68153844
$$$$
G=-735.73377479
$$$$
\mathrm{n}_{\text {imag }}=0
$$

C $0.57256-0.000732 .86833$

H 1.073500 .758373 .48765

C $1.49294-1.214562 .76950$

H 1.59046 -1.95195 3.55819

C $2.20415-1.219081 .63994$

H 2.95461 -1.96058 1.39161

C 0.607030 .378781 .42326

C - 0.494300 .465110 .71474

C - $0.494300 .46511-0.71474$ 
C $0.607030 .37878-1.42326$

C $0.57256-0.00073-2.86833$

H $1.073510 .75836-3.48765$

C $1.49293-1.21458-2.76950$

C $2.20413-1.21909-1.63993$

H 1.59044 -1.95197 -3.55818

H 2.95459 -1.96061 -1.39159

C -1.90625 0.42557 1.19176

H -2.23012 -0.62509 1.13384

C -1.90625 0.42557-1.19176

H -2.23012 -0.62509-1.13385

C -2.63876 1.124760.00000

H -3.717600.960860.00000

H -2.44632 2.202240.00000

C $1.931760 .01950-0.78660$

H 2.689710 .75734 -1.08133

C 1.931760 .019500 .78660

H 2.689710 .757361 .08133

C -1.90021 $0.77836-2.67575$

H -2.88178 $0.64914-3.13818$

H -1.60118 $1.82135-2.82147$

C -0.88106 -0.17501-3.36566

H -0.92396 -0.03748 -4.44850

H -1.20207-1.20375-3.16980

C -1.90022 0.778352 .67575

H -1.601201.821342.82148

H -2.881790.64911 3.13818

C -0.88106 -0.17502 3.36566

H -1.20206 -1.20376 3.16980

H -0.92396 -0.03750 4.44850

2'B $\left(C_{s}, 2 \mathrm{nN}\right)$ 


$$
\begin{aligned}
& E=-736.01766596 \\
& H=-735.66131692 \\
& G=-735.71350557 \\
& \mathrm{n}_{\text {imag }}=0
\end{aligned}
$$

C $0.58258-0.000122 .89858$

H 1.222680 .817573 .26962

C $1.32936-1.310593 .12572$

H 1.43391 -1.71387 4.16413

C $1.90442-1.735711 .98181$

H 2.54226 -2.60896 1.90678

C 0.565160 .014561 .40822

C - 0.511170 .302480 .71578

C - $0.511170 .30248-0.71578$

C $0.565160 .01456-1.40822$

C $0.58258-0.00012-2.89858$

H $1.222680 .81758-3.26962$

C $1.32936-1.31059-3.12572$

C $1.90442-1.73571-1.98181$

H $1.43391-1.71387-4.16413$

H 2.54226 -2.60895 -1.90678

C -1.81506 0.82694 1.21334

H - 2.52589 -0.01176 1.24991

C - $-1.815060 .82694-1.21334$

H -2.52589 -0.01176 -1.24991

C - 2.250781 .712400 .00000

H -3.31621 1.949240 .00000

H -1.68773 2.651960.00000

C $1.76905-0.68328-0.82566$

H $2.64337-0.04817$-1.01873

C $1.76905-0.683290 .82566$ 


$$
\begin{aligned}
& \text { H 2.64337 -0.04817 1.01874 } \\
& \text { C -1.60789 } 1.31444-2.64760 \\
& \text { H -2.55616 } 1.53058 \text {-3.14572 } \\
& \text { H -1.02408 2.24161 -2.64487 } \\
& \text { C - } 0.840650 .22024-3.44391 \\
& \text { H -0.80686 0.49124 -4.50210 } \\
& \text { H -1.40113 -0.71837 -3.37446 } \\
& \text { C - } 1.607881 .314442 .64760 \\
& \text { H -1.02408 2.24161 2.64487 } \\
& \text { H -2.55615 } 1.530593 .14572 \\
& \text { C - } 0.840650 .220243 .44391 \\
& \text { H -1.40114 -0.71836 3.37446 } \\
& \text { H -0.80686 0.49124 4.50210 } \\
& 3 \mathrm{~A}\left(C_{1}, 0 \mathrm{nN}\right) \\
& E=-812.23149433 \\
& H=-811.86097641 \\
& G=-811.9175878 \\
& \mathrm{n}_{\text {imag }}=0 \\
& \text { C -0.01176 -0.03239 2.91869 } \\
& \text { H -0.57339 -0.65247 } 3.63074 \\
& \text { C } 1.46855-0.115313 .22447 \\
& \text { H } 1.894890 .326734 .11767 \\
& \text { C } 2.15023-0.780092 .30231 \\
& \text { H } 3.21579-0.971462 .33616 \\
& \text { C -0.05696 -0.61097 } 1.52232 \\
& \text { C -0.93855 -0.39093 } 0.55519 \\
& \text { C -0.56514-0.54271 -0.87372 } \\
& \text { C } 0.67618-0.85696-1.22885 \\
& \text { C } 1.42830-0.47298-2.48593 \\
& \text { H } 1.38120 \text {-1.26433 -3.24659 }
\end{aligned}
$$




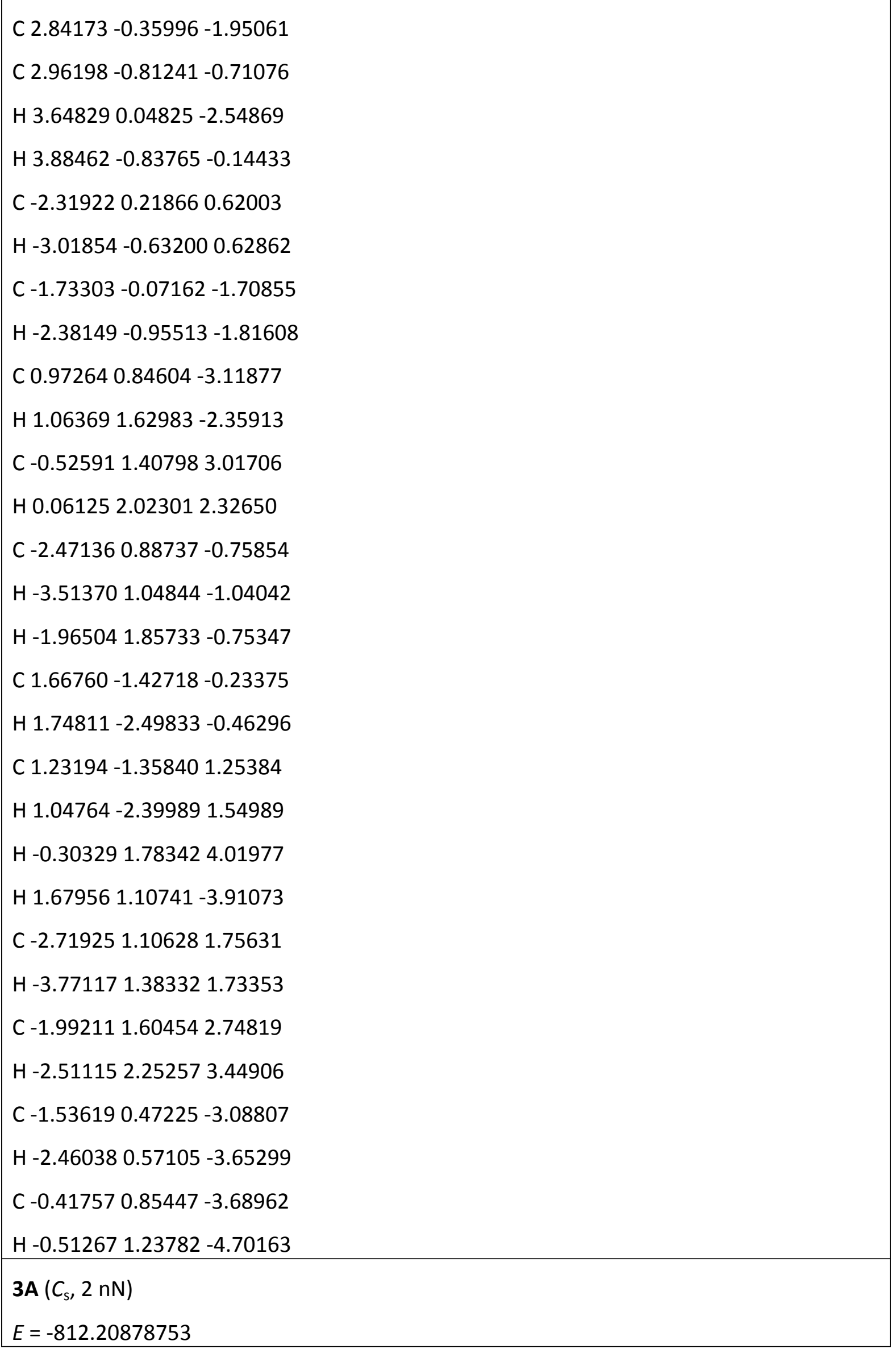




$$
\begin{aligned}
& H=-811.83978934 \\
& G=-811.89569879 \\
& \mathrm{n}_{\text {imag }}=0
\end{aligned}
$$

C $0.74066-0.000192 .91542$

H $0.72863-0.930683 .50039$

C 2.086820 .680623 .04733

H 2.408601 .002894 .06849

C 2.723830 .744611 .87102

H 3.701871 .185411 .71783

C $0.65957-0.304871 .43729$

C - $0.41960-0.627790 .73757$

C $-0.41962-0.62778-0.73758$

C $0.65959-0.30500-1.43732$

C $0.74066-0.00041-2.91546$

H $0.72825-0.93091$-3.50039

C $2.087030 .67994-3.04753$

C $2.724110 .74394-1.87127$

H $2.408861 .00194-4.06876$

H 3.702281 .18447 -1.71819

C -1.81444 -0.96329 1.21264

H -1.85328 -2.06140 1.29444

C -1.81451 -0.96305 -1.21266

H -1.85344 -2.06114 -1.29471

C -0.37717 0.91032 -3.43749

H -0.34796 $1.84386-2.86475$

C - 0.377410 .910183 .43756

H -0.34856 1.843742 .86484

C -2.68397 -0.58761 0.00005

H -3.65293 -1.09105 0.00002

H -2.86118 0.492400 .00017 

C $2.03329-0.13884-0.80994$
H 2.52729 -1.10021 -0.99959
C $2.03326-0.138610 .80991$
H 2.52744 -1.09983 0.99985
H -0.13741 1.186484 .46894
H -0.13717 1.18657-4.46889
C -2.34768 -0.41836 2.50012
H -3.37992 -0.70774 2.68779
C -1.77158 0.35040 3.41520
H -2.390130.63404 4.26266
C - $2.34773-0.41777-2.50000$
H -3.38007 -0.70685 -2.68762
C -1.77152 0.35097 -3.41502
H -2.39007 $0.63491-4.26238$

3B $\left(C_{\mathrm{s}}, 0 \mathrm{nN}\right)$

$E=-812.21354178$

$H=-811.84352719$

$G=-811.89983438$

$\mathrm{n}_{\text {imag }}=0$

C $0.83535-0.001982 .79236$

H 1.01033 -1.08444 2.87078

C 2.174430 .706362 .84537

H 2.843620 .633163 .69487

C 2.455601 .360901 .71941

H 3.375121 .904621 .53788

C 0.403070 .325561 .39967

C -0.49588-0.35808 0.72236

C -0.49588 $-0.35808-0.72236$

C $0.403070 .32556-1.39967$

C $0.83535-0.00198-2.79236$ 


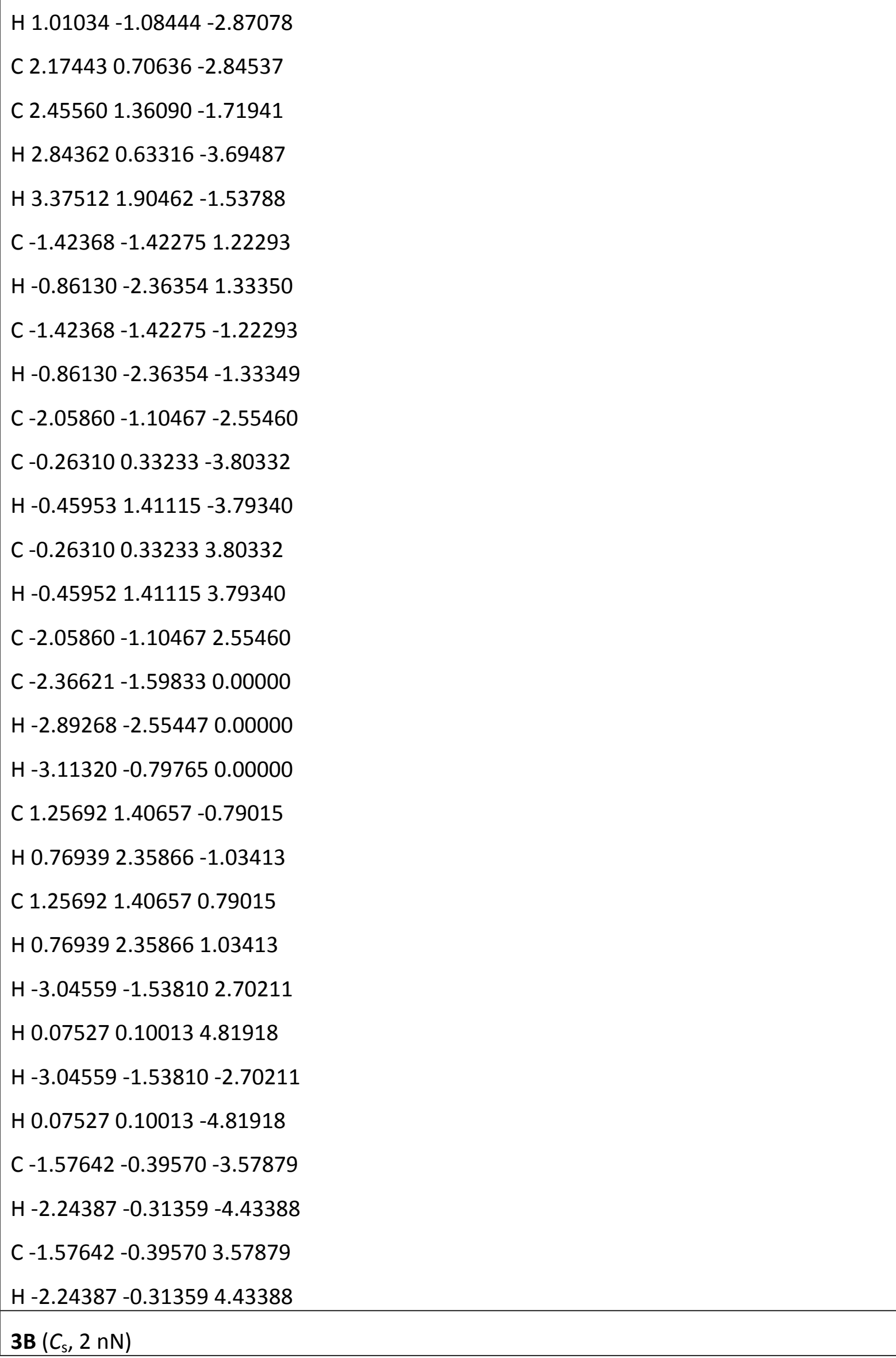




$$
\begin{aligned}
& E=-812.19682958 \\
& H=-811.82831919 \\
& G=-811.88446532 \\
& n_{\text {imag }}=0
\end{aligned}
$$

C 0.764350 .001152 .89903

H 0.85223 -1.06315 3.15646

C 2.103400 .677853 .10980

H 2.515980 .742914 .14778

C 2.597431 .151671 .95451

H 3.545261 .667741 .85603

C 0.550660 .152791 .42073

C $-0.37020-0.489280 .72565$

C $-0.37020-0.48928-0.72564$

C $0.550660 .15281-1.42073$

C $0.764350 .00117-2.89903$

H 0.85226 -1.06312 -3.15646

C $2.103380 .67792-3.10980$

C $2.597401 .15174-1.95451$

H 2.51595 0.74300 -4.14778

H $3.545221 .66784-1.85604$

C -1.46582 -1.38760 1.23040

H -1.05741 -2.39656 1.39446

C $-1.46581-1.38762-1.23040$

H -1.05738 -2.39657 -1.39443

C - $2.15241-0.94838-2.49714$

C - $0.415750 .55565-3.69975$

H -0.54216 1.62147 -3.47375

C - 0.415740 .555663 .69974

H -0.54211 1.621493 .47374

C - $2.15243-0.948332 .49713$ 


$$
\begin{aligned}
& \text { C -2.40357 -1.45649 } 0.00000 \\
& \text { H -3.04057 -2.34304 } 0.00000 \\
& \text { H -3.05313 -0.57498 -0.00001 } \\
& \text { C } 1.532811 .13616-0.82508 \\
& \text { H } 1.073032 .11778-0.98663 \\
& \text { C } 1.532821 .136140 .82509 \\
& \text { H } 1.073062 .117770 .98667 \\
& \text { H -3.15500 -1.35567 2.61317 } \\
& \text { H -0.18434 } 0.505444 .76963 \\
& \text { H -3.15497 -1.35575 -2.61319 } \\
& \text { H -0.18435 0.50543 -4.76964 } \\
& \text { C -1.73101 -0.15400 -3.48009 } \\
& \text { H -2.44897 } 0.01996-4.27829 \\
& \text { C -1.73102 -0.15394 } 3.48007 \\
& \text { H -2.44898 } 0.020054 .27826
\end{aligned}
$$$$
\text { 3'A }\left(C_{\mathrm{s}}, 0 \mathrm{nN}\right)
$$$$
E=-812.20484804
$$$$
H=-811.83466142
$$$$
G=-811.89104984
$$$$
\mathrm{n}_{\text {imag }}=0
$$

C $0.85618-0.005442 .62456$

H 1.101761 .040612 .39210

C $2.17257-0.742392 .68605$

H $2.90056-0.567253 .47011$

C $2.36526-1.528221 .63231$

H 3.26501 -2.10201 1.44477

C $0.22291-0.595231 .39196$

C -0.82865 -0.116190.73838

C -0.82865 -0.11619-0.73838

C $0.22291-0.59523-1.39196$ 


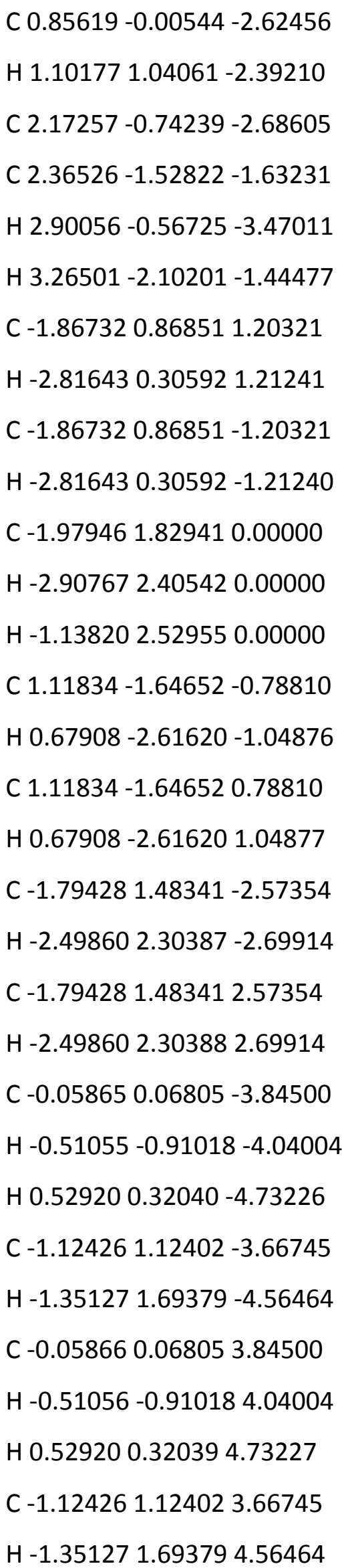




$$
\begin{aligned}
& 3^{\prime} \mathrm{A}\left(C_{\mathrm{s}}, 2 \mathrm{nN}\right) \\
& E=-812.1827303 \\
& H=-811.81427335 \\
& G=-811.87030177 \\
& \mathrm{n}_{\text {imag }}=0
\end{aligned}
$$

C $0.84757-0.034052 .83635$

H 1.195171 .008402 .84694

C $2.06981-0.892343 .07854$

H $2.48120-0.969054 .11649$

C $2.49752-1.448681 .93613$

H 3.38433 -2.06466 1.84362

C $0.45462-0.377841 .41996$

C - 0.577300 .103120 .73566

C - $0.577810 .10245-0.73574$

C $0.45351-0.37933-1.42021$

C $0.84671-0.03496-2.83631$

H $1.193841 .00764-2.84606$

C $2.06965-0.89223-3.07852$

C $2.49702-1.44913-1.93622$

H $2.48261-0.96665$-4.11602

H $3.38513-2.06314-1.84306$

C - 1.760380 .921151 .20025

H -2.608860.21585 1.21147

C - $1.760370 .92126-1.20037$

H -2.60921 $0.21641-1.21187$

C - 2.014311 .848260 .00000

H -3.01947 2.27630 -0.00002

H -1.289132.668720.00011

C $1.42600-1.37713-0.82534$

H $0.93582-2.34358-0.98026$ 


$$
\begin{aligned}
& \text { C } 1.42635-1.376600 .82543 \\
& \text { H } 0.93551-2.342590 .98124 \\
& \text { C -1.76264 } 1.56277-2.55867 \\
& \text { H - } 2.371002 .46154-2.63025 \\
& \text { C - } 1.763011 .562502 .55856 \\
& \text { H - } 2.371142 .461442 .63006 \\
& \text { C }-0.30924-0.08633-3.84015 \\
& \text { H -0.86871 -1.01951 -3.71545 } \\
& \text { H } 0.09161-0.09521-4.85714 \\
& \text { C -1.21391 } 1.11174-3.68213 \\
& \text { H -1.40137 } 1.68712-4.58419 \\
& \text { C -0.30875 -0.08598 } 3.83979 \\
& \text { H -0.86770 -1.01942 } 3.71466 \\
& \text { H } 0.09177-0.094974 .85691 \\
& \text { C -1.21399 1.111673.68192 } \\
& \text { H -1.40106 } 1.687324 .58388
\end{aligned}
$$$$
\text { 3'B }\left(C_{s}, 0 \mathrm{nN}\right)
$$$$
E=-812.22931797
$$$$
H=-811.85955621
$$$$
G=-811.91504951
$$$$
\mathrm{n}_{\text {imag }}=0
$$

C 0.825010 .001102 .88291

H 1.071480 .913303 .44930

C $2.09601-0.818742 .74059$

H 2.47210 -1.45893 3.53004

C $2.70278-0.591991 .58031$

H 3.65237 -1.01803 1.28053

C 0.596080 .352921 .43426

C - 0.524810 .282160 .73465

C $-0.524810 .28216-0.73465$ 


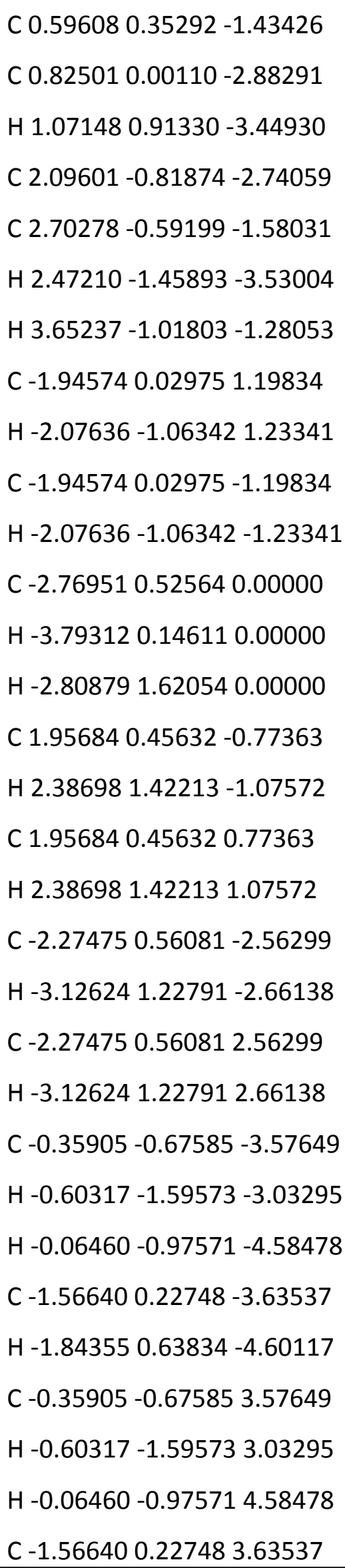




\section{H - 1.843550 .638344 .60117}

3'B $\left(C_{5}, 2 \mathrm{nN}\right)$

$E=-812.20901188$

$H=-811.84064526$

$G=-811.89611409$

$\mathrm{n}_{\text {imag }}=0$

C $0.79912-0.000062 .93256$

H 1.086100 .995333 .31031

C $2.00920-0.905613 .04947$

H $2.30483-1.257294 .06877$

C $2.61823-1.041401 .86182$

H 3.53605 -1.59132 1.69055

C 0.625430 .089431 .43609

C -0.49577 0.16810 0.73599

C -0.49577 0.16810 -0.73599

C $0.625430 .08943-1.43609$

C $0.79912-0.00006-2.93256$

H $1.086100 .99533-3.31031$

C $2.00920-0.90561-3.04947$

C $2.61823-1.04140-1.86182$

H $2.30483-1.25729-4.06877$

H 3.53605 -1.59132 -1.69055

C -1.93573 0.24941 1.20775

H - $2.30120-0.786481 .28770$

C -1.93573 0.24941 -1.20775

H - 2.30120 -0.78648 -1.28770

C -2.640170.880440.00000

H -3.71860 0.709250.00000

H -2.46770 1.962570 .00000

C $1.99445-0.09380-0.80322$ 


$$
\begin{aligned}
& \text { H } 2.533340 .84019-1.01660 \\
& \text { C } 1.99445-0.093800 .80322 \\
& \text { H } 2.533340 .840191 .01660 \\
& \text { C - } 2.178750 .91697-2.53136 \\
& \text { H -2.94451 } 1.68719-2.56346 \\
& \text { C -2.178750.916972.53136 } \\
& \text { H -2.94451 } 1.687192 .56346 \\
& \text { C }-0.44400-0.44235-3.70663 \\
& \text { H -0.79570 -1.39359 -3.29138 } \\
& \text { H -0.17124 -0.63559-4.74686 } \\
& \text { C -1.53613 } 0.59351-3.64568 \\
& \text { H -1.78215 } 1.11908-4.56327 \\
& \text { C - } 0.44400-0.442353 .70663 \\
& \text { H -0.79570 -1.39359 } 3.29138 \\
& \text { H -0.17124 -0.63559 4.74686 } \\
& \text { C - } 1.536130 .593513 .64568 \\
& \text { H -1.78215 1.11908 4.56327 }
\end{aligned}
$$

4A $\left(C_{\mathrm{s}}, 0 \mathrm{nN}\right)$

$E=-994.87082469$

$H=-994.51064169$

$G=-994.57092061$

$\mathrm{n}_{\text {imag }}=0$

C $-0.90433-0.403902 .75869$

H -1.161360.24200 3.60836

C -1.93662 -1.50131 2.62298

H -2.03559 -2.28481 3.36457

C - $2.68386-1.374671 .53627$

H -3.49469 -2.03759 1.26187

C -1.04707 0.29700 1.42646

C - 0.096030 .918560 .74132 


$$
\begin{aligned}
& \text { C }-0.095960 .91846-0.74127 \\
& \text { C }-1.047030 .29698-1.42644 \\
& \text { C -0.90434 }-0.40393-2.75869 \\
& \text { H -1.16153 } 0.24195-3.60832 \\
& \text { C -1.93649 -1.50144 -2.62281 } \\
& \text { C - } 2.68413-1.37440-1.53643 \\
& \text { H }-2.03514-2.28525-3.36412
\end{aligned}
$$$$
\text { H -3.49495 -2.03731 -1.26199 }
$$$$
\text { C } 1.250271 .437541 .18907
$$$$
\text { H } 1.145212 .531681 .19160
$$$$
\text { C } 1.250371 .43738-1.18895
$$$$
\text { H } 1.145292 .53151 \text {-1.19161 }
$$$$
\text { C } 0.50255-0.97403-2.97311
$$$$
\text { H } 0.80903-1.49894-2.06342
$$$$
\text { C } 0.50260-0.974002 .97284
$$$$
\text { H } 0.80909-1.498452 .06288
$$$$
\text { C } 2.159501 .092540 .00012
$$$$
\text { H } 3.094681 .655080 .00012
$$$$
\text { H } 2.391830 .023870 .00021
$$$$
\text { C }-2.35211-0.11138-0.77904
$$$$
\text { H -3.09851 } 0.63740-1.07293
$$$$
\text { C -2.35206-0.111510.77902 }
$$$$
\text { H -3.098570.63709 } 1.07307
$$$$
\text { H } 0.50137 \text {-1.69319 } 3.79129
$$$$
\text { H } 0.50114 \text {-1.69288 -3.79187 }
$$$$
\text { N } 1.753771 .10796-2.50177
$$$$
\text { H } 2.448041 .74011-2.86788
$$$$
\text { N } 1.753561 .108182 .50195
$$$$
\text { H } 2.448121 .740092 .86792
$$$$
\text { C } 1.537370 .06038-3.34666
$$$$
\text { C } 1.537270 .060443 .34667
$$ 
O $2.15314-0.01085-4.39216$
O $2.15291-0.010844 .39224$

$4 \mathrm{~A}\left(C_{\mathrm{s}}, 2 \mathrm{nN}\right)$

$E=-994.84575556$

$H=-994.48718799$

$G=-994.54739427$

$\mathrm{n}_{\text {imag }}=0$

C $-0.93712-0.272332 .90131$

H -1.16265 0.603083 .52521

C -2.03747 -1.30315 3.03702

H -2.24482 -1.70985 4.05681

C -2.64969-1.52319 1.86661

H -3.47027 -2.21423 1.71617

C -0.97905 0.073331 .43460

C -0.017370 .658270 .73673$

C -0.01740 0.65831-0.73680

C -0.97914 $0.07343-1.43468$

C - $0.93733-0.27224-2.90141$

H -1.16291 0.60312 -3.52536

C -2.03768 -1.30309 -3.03700

C -2.64983-1.52311-1.86655

H -2.24510 - 1.70985 -4.05675

H -3.47038 -2.21415 -1.71600

C 1.250721 .335061 .19777

H 1.010602 .408421 .23989

C $1.250671 .33520-1.19780$

H 1.010532 .40856 -1.23981

C $0.41505-0.85448-3.32730$

H 0.70505 -1.62169-2.60345

C $0.41522-0.854643 .32724$ 


$$
\begin{aligned}
& \text { H } 0.70527-1.621892 .60344 \\
& \text { C } 2.187371 .13097-0.00003 \\
& \text { H } 3.036621 .817360 .00002 \\
& \text { H } 2.565280 .10520-0.00010 \\
& \text { C -2.24990 -0.47013 -0.81002 } \\
& \text { H -2.99807 } 0.30827-1.00472 \\
& \text { C -2.24985 }-0.470190 .81005 \\
& \text { H -2.99798 } 0.308211 .00483 \\
& \text { H } 0.33079-1.336934 .30095 \\
& \text { H } 0.33072-1.33679-4.30101 \\
& \text { N } 1.849091 .00016-2.47217 \\
& \text { H } 2.623331 .59159-2.73375 \\
& \text { N } 1.849230 .999992 .47213 \\
& \text { H } 2.623241 .591662 .73385 \\
& \text { C } 1.553110 .12921-3.47873 \\
& \text { C } 1.553250 .129053 .47871 \\
& \text { O } 2.227840 .13954-4.49094 \\
& \text { O } 2.227810 .139494 .49103 \\
& \hline
\end{aligned}
$$$$
\text { 4B }\left(C_{\mathrm{s}}, 0 \mathrm{nN}\right)
$$$$
E=-994.84465741
$$$$
H=-994.48529341
$$$$
G=-994.54610448
$$$$
\mathrm{n}_{\text {imag }}=0
$$

C -1.077810.181242.77178

H -1.04597 1.279992 .81132

C $-2.53008-0.248972 .85500$

H -3.15640 -0.03510 3.71268

C -2.95499-0.84108 1.73912

H -3.96787 -1.18912 1.57719

C - $0.73864-0.270391 .39380$ 


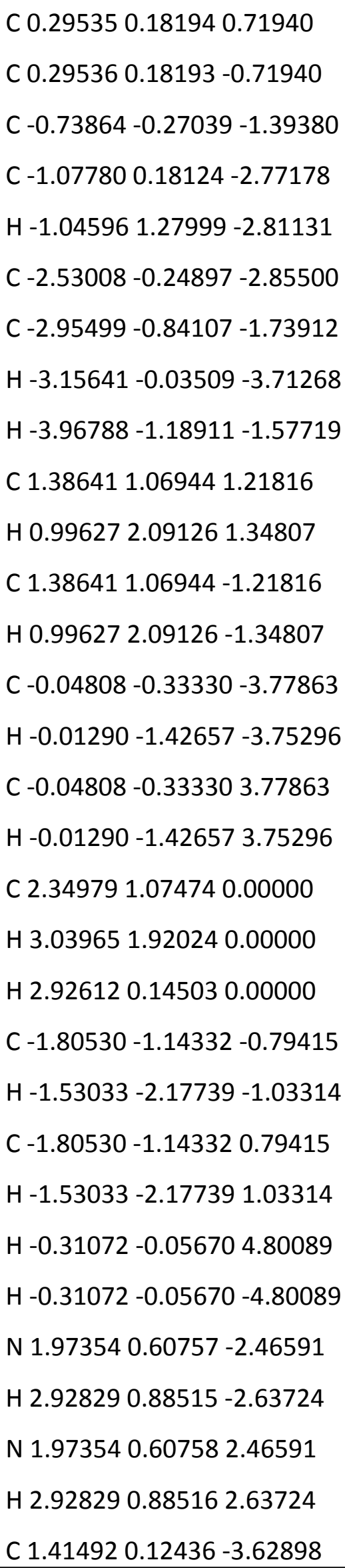




$$
\begin{aligned}
& \text { C } 1.414920 .124363 .62898 \\
& \text { O } 2.134060 .00028-4.60104 \\
& \text { O } 2.134060 .000294 .60104
\end{aligned}
$$$$
\text { 4B }\left(C_{\mathrm{s}}, 2 \mathrm{nN}\right)
$$$$
E=-994.82815592
$$$$
H=-994.47018511
$$$$
G=-994.53078558
$$$$
\mathrm{n}_{\text {imag }}=0
$$

C - 1.045610 .042762 .88610 H -1.10723 1.115653 .11584

C $-2.40419-0.588793 .12078$

H -2.80731 -0.63202 4.16309

C -2.92972 -1.05092 1.97440

H -3.89716 -1.53126 1.89165

C -0.84811-0.15732 1.41655

C 0.120040 .408160 .72241

C $0.120030 .40816-0.72241$

C -0.84809-0.15739-1.41653

C - $-1.045620 .04263-2.88610$

H -1.10744 $1.11550-3.11585$

C -2.40409-0.58918 -3.12079

C -2.92959-1.05133 -1.97440

H -2.80715 -0.63255 -4.16311

H -3.89694 -1.53184 -1.89167

C 1.226321 .281251 .22991

H 0.831282 .287621 .43452

C $1.226241 .28136-1.22988$

H $0.831152 .28773-1.43436$

C $0.12563-0.53618-3.68123$

H 0.26752 -1.58838 -3.41446 


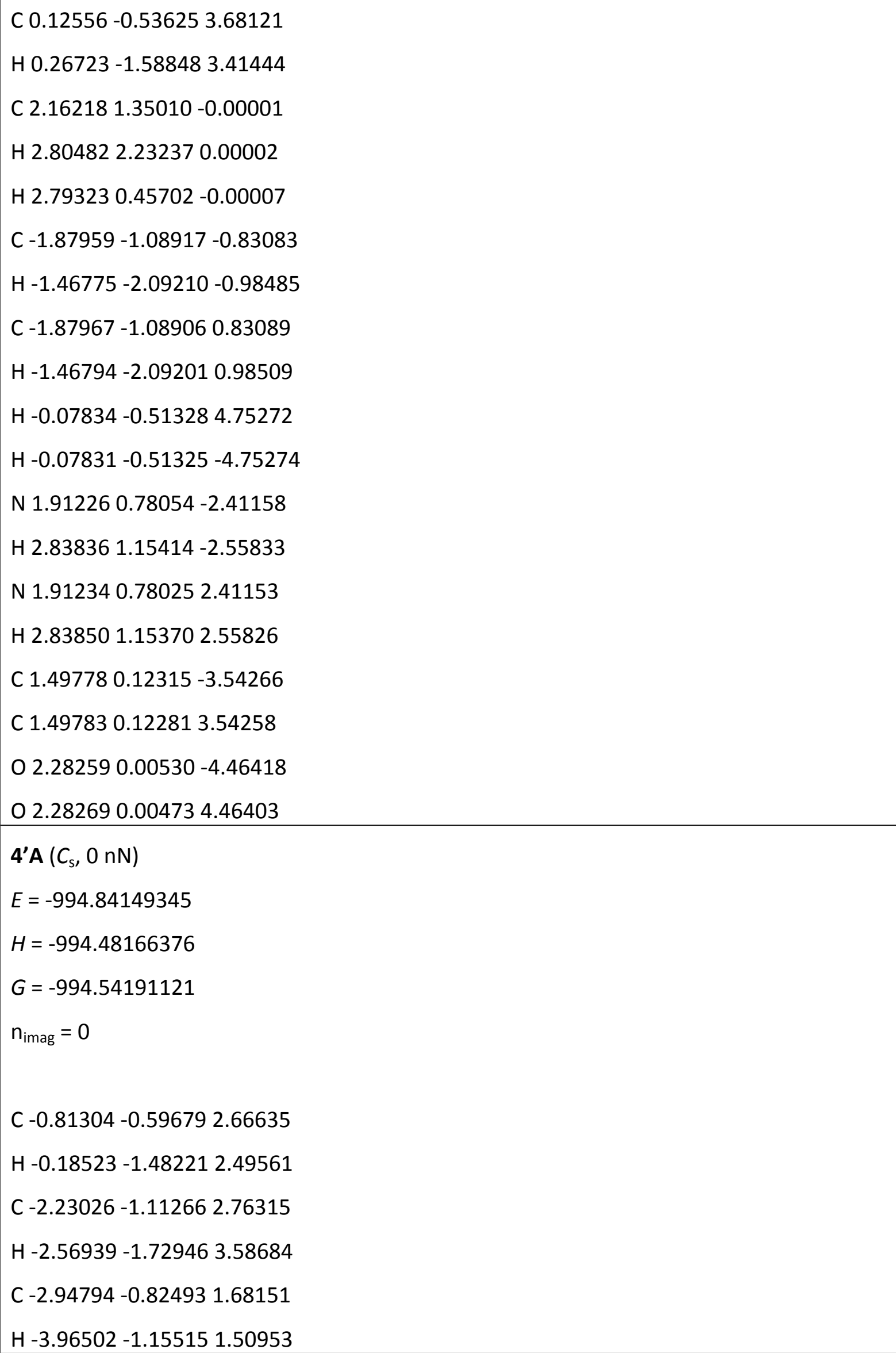



C -0.855150 .184611 .38587$
C 0.189580 .672870 .73536
C $0.189580 .67287-0.73536$
C $-0.855150 .18461-1.38587$
C - $0.81305-0.59678-2.66635$
H -0.18524 -1.48221 -2.49561
C -2.23026-1.11265 -2.76315
C -2.94795 -0.82493 -1.68151
H -2.56939 -1.72945 -3.58685
H -3.96502 -1.15514 -1.50954
C 1.605330 .837171 .21068
H 1.784321 .919291 .31144
C $1.605330 .83717-1.21068$
H $1.784321 .91929-1.31144$
C 2.419540 .341710 .00000
H 3.449970 .704920 .00000
H $2.42831-0.751630 .00000$
C -2.23619 0.16723 -0.78891
H -2.68313 $1.13627-1.03831$
C -2.236190.167230.78891
H -2.683131.136261.03831
H $2.986630 .12412-2.58180$
H 2.986630 .124132 .58180
C $-0.146080 .17240-3.80437$
H -0.48936 1.21084-3.82380
H -0.36112 -0.26274 -4.78016
C - 0.146080 .172393 .80437
H -0.48936 1.210833 .82380
H -0.36112 -0.26274 4.78016
N $1.986300 .17579-2.45080$
N 1.986300 .175792 .45080 


$$
\begin{aligned}
& \text { C } 1.374370 .14404-3.68827 \\
& \text { C } 1.374370 .144053 .68827 \\
& \text { O } 2.05892-0.00145-4.67997 \\
& \text { O } 2.05892-0.001424 .67997 \\
& \hline
\end{aligned}
$$$$
\text { 4'A }\left(C_{\mathrm{s}}, 2 \mathrm{nN}\right)
$$$$
E=-994.82153363
$$$$
H=-994.46336612
$$$$
G=-994.52319657
$$$$
\mathrm{n}_{\mathrm{imag}}=0
$$

C $-0.83723-0.498112 .83781$

H -0.29570 -1.45415 2.86681

C -2.28811 -0.83590 3.11044

H -2.60521 -1.07899 4.15521

C -2.99999 -0.82130 1.97307

H -4.04694 -1.08900 1.89843

C $-0.86090-0.013711 .41379$

C 0.180500 .437860 .73058

C $0.180510 .43781-0.73057$

C -0.86080 -0.01397-1.41377

C -0.83701 -0.49846 -2.83774

H -0.29509 -1.45428 -2.86673

C -2.28777 -0.83683 -3.11029

C -2.99967-0.82217 -1.97293

H -2.60479 -1.08026 -4.15501

H -4.04654 -1.09016 -1.89825

C 1.574530 .756961 .20612

H 1.638521 .852841 .29955

C $1.574480 .75713-1.20609$

H 1.638331 .85304 -1.29929

C $2.435450 .34678-0.00004$ 


$$
\begin{aligned}
& \text { H } 3.421930 .81595-0.00002 \\
& \text { H } 2.55592-0.74063-0.00012 \\
& \text { C -2.25265 -0.08854 -0.83241 } \\
& \text { H -2.64913 } 0.92088-0.97563 \\
& \text { C -2.25272 -0.088220.83236 } \\
& \text { H -2.64903 } 0.921320 .97517 \\
& \text { H } 3.00878-0.00376-2.52536 \\
& \text { H } 3.00869-0.004782 .52508 \\
& \text { C - } 0.076110 .44769-3.77124 \\
& \text { H -0.30692 } 1.49006 \text {-3.53097 } \\
& \text { H -0.33515 0.27887 -4.81594 } \\
& \text { C - } 0.075900 .447713 .77130 \\
& \text { H -0.30629 } 1.490203 .53113 \\
& \text { H -0.33493 0.27890 4.81600 } \\
& \text { N } 2.013290 .13941-2.44361 \\
& \text { N } 2.013260 .138882 .44347 \\
& \text { C } 1.424750 .20750-3.68371 \\
& \text { C } 1.424840 .206873 .68364 \\
& \text { O } 2.08486-0.00434-4.67973 \\
& \text { O } 2.08479-0.006274 .67949
\end{aligned}
$$

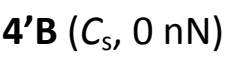$$
E=-994.87387264
$$$$
H=-994.5141963
$$$$
G=-994.574019
$$$$
\mathrm{n}_{\text {imag }}=0
$$

C $-0.96774-0.342492 .87829$

H -0.82654 -1.26059 3.46821

C -2.46076 -0.09321 2.74053

H -3.053890.35305 3.52951

C -2.93598-0.55599 1.58835 


$$
\begin{aligned}
& \text { H -3.97908 -0.54397 } 1.29775 \\
& \text { C -0.63894 -0.59540 } 1.43266 \\
& \text { C } 0.34207-0.059920 .72965 \\
& \text { C } 0.34207-0.05992-0.72965 \\
& \text { C }-0.63895-0.59538-1.43267 \\
& \text { C -0.96775 -0.34246 - } 2.87829 \\
& \text { H -0.82660 -1.26057 -3.46821 } \\
& \text { C - } 2.46076-0.09312-2.74051 \\
& \text { C }-2.93598-0.55591-1.58832 \\
& \text { H -3.05389 0.35318 -3.52946 } \\
& \text { H -3.97907 -0.54386 -1.29771 } \\
& \text { C } 1.508670 .784361 .18919 \\
& \text { H } 1.175781 .831621 .22467 \\
& \text { C } 1.508680 .78434-1.18920 \\
& \text { H } 1.175801 .83160 \text {-1.22471 } \\
& \text { C } 2.476120 .675250 .00000 \\
& \text { H } 3.245681 .448210 .00000 \\
& \text { H } 2.95422-0.310420 .00001 \\
& \text { C }-1.84453-1.23526-0.77627 \\
& \text { H -1.86078 -2.29148 -1.07808 } \\
& \text { C - }-1.84452-1.235290 .77626 \\
& \text { H -1.86074 -2.29152 } 1.07803 \\
& \text { H } 3.007550 .10931-2.54656 \\
& \text { H } 3.007550 .109392 .54658 \\
& \text { C - } 0.138060 .76513-3.53210 \\
& \text { H -0.25907 } 1.69540-2.97010 \\
& \text { H -0.47507 } 0.93741-4.55335 \\
& \text { C -0.13809 0.76513 } 3.53209 \\
& \text { H -0.25913 1.695392.97007 } \\
& \text { H -0.475110.93741 } 4.55334 \\
& \text { N } 2.056170 .43019-2.47551
\end{aligned}
$$




$$
\begin{aligned}
& \text { N } 2.056160 .430242 .47551 \\
& \text { C } 1.331020 .37172-3.62984 \\
& \text { C } 1.331010 .371783 .62984 \\
& \text { O } 1.828000 .00245-4.67339 \\
& \text { O } 1.828010 .002584 .67341
\end{aligned}
$$

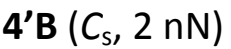$$
E=-994.85307675
$$$$
H=-994.49492861
$$$$
G=-994.55461171
$$$$
\mathrm{n}_{\text {imag }}=0
$$

C -0.88995 -0.44700 2.92738

H -0.56368 -1.41975 3.32878

C -2.39944 -0.37135 3.06080

H -2.83099 -0.24089 4.08333

C -2.99421 -0.61005 1.88121

H -4.06474 -0.66927 1.72636

C -0.72506 -0.44854 1.43245

C 0.231510 .131380 .73045

C $0.231510 .13138-0.73044$

C $-0.72503-0.44859-1.43243$

C $-0.88991-0.44708-2.92737$

H -0.56351 -1.41980 -3.32876

C - $2.39939-0.37159-3.06083$

C $-2.99418-0.61027-1.88126$

H -2.83093 -0.24124-4.08339

H -4.06471 -0.66958 -1.72643

C 1.454000 .885601 .20222

H 1.185271 .948331 .29477

C $1.453970 .88564-1.20220$

H $1.185211 .94837-1.29469$ 


$$
\begin{aligned}
& \text { C } 2.399900 .754520 .00000 \\
& \text { H } 3.197811 .498930 .00000 \\
& \text { H } 2.84399-0.24675 \text {-0.00003 } \\
& \text { C -1.96393-1.05987 -0.80746 } \\
& \text { H -1.89672 -2.13575 -1.01786 } \\
& \text { C -1.96396-1.05980 0.80748 } \\
& \text { H -1.89682 -2.13567 1.01798 } \\
& \text { H } 3.021730 .22778-2.47559 \\
& \text { H } 3.021740 .227532 .47553 \\
& \text { C -0.09409 0.64201-3.65038 } \\
& \text { H -0.29989 } 1.61237-3.18845 \\
& \text { H -0.38513 } 0.69512-4.69866 \\
& \text { C -0.094020.64199 } 3.65040 \\
& \text { H -0.29973 } 1.612393 .18850 \\
& \text { H -0.385040.695114.69869 } \\
& \text { N 2.03526 } 0.42638-2.44176 \\
& \text { N } 2.035290 .426242 .44174 \\
& \text { C } 1.396480 .34188-3.64330 \\
& \text { C } 1.396510 .341713 .64329 \\
& \text { O } 1.988270 .00511-4.64832 \\
& \text { O } 1.988270 .004704 .64825
\end{aligned}
$$$$
5 \mathrm{~A}\left(C_{\mathrm{s}}, 0 \mathrm{nN}\right)
$$$$
E=-814.70317332
$$$$
H=-814.28451929
$$$$
G=-814.34218023
$$$$
\mathrm{n}_{\text {imag }}=0
$$

C 0.875660 .001712 .88158

H 1.12140 -0.91034 3.45202

C 2.139450 .831272 .75072

H 2.502641 .456763 .55787 


\section{2.732570 .664751 .57684}

H 3.663541 .126771 .27286

C $0.64112-0.381721 .43504$

C $-0.47857-0.575000 .74414$

C -0.47863 $-0.57480-0.74411$

C $0.64102-0.38114-1.43504$

C $0.875990 .00226-2.88157$

H 1.12402 -0.90963 -3.45129

C $2.138250 .83397-2.74946$

C $2.732470 .66545-1.57642$

H 2.500581 .46098 -3.55581

H $3.663301 .12756-1.27216$

C -1.90077 -0.75767 1.23892

H -1.99687 -1.81817 1.51117

C -1.90098 -0.75644-1.23866

H -1.99764 -1.81662 -1.51194

C -2.37791 0.07439-2.42567

H -2.41651 1.12666 -2.12044

C $-0.235040 .72572-3.63186$

H -0.40186 1.70289-3.16518

C -0.235390.72735 3.62964

H -0.40297 1.702673 .15932

C - 2.378580 .072032 .42645

H -2.42028 1.124032 .12078

C - $2.76278-0.484610 .00032$

H -3.69057 -1.06054 0.00007

H -3.044390.574440.00093

C $2.00465-0.38496-0.76938$

H 2.46556 -1.34007 -1.06260

C $2.00469-0.385240 .76933$

H 2.46565 -1.340461.06213 


$$
\begin{aligned}
& \text { H -3.41215 -0.22472 2.63459 } \\
& \text { H } 0.128570 .930854 .64191 \\
& \text { H -3.41236 -0.21978 -2.63294 } \\
& \text { H } 0.128870 .92529-4.64498 \\
& \text { C -1.56047 -0.02416 -3.70745 } \\
& \text { H -2.15351 } 0.39578-4.52474 \\
& \text { H -1.38419 -1.07498 -3.96749 } \\
& \text { C -1.56008 -0.02347 3.70784 } \\
& \text { H -2.152940.39765 4.52467 } \\
& \text { H -1.38253 -1.07353 } 3.97006 \\
& 5 \mathrm{~A}\left(C_{\mathrm{s}}, 2 \mathrm{nN}\right) \\
& E=-814.68373071 \\
& H=-814.26660829 \\
& G=-814.32356609 \\
& \mathrm{n}_{\text {imag }}=0
\end{aligned}
$$

C 0.846820 .005462 .94124

H $1.12466-0.979573 .35500$

C 2.045680 .926373 .04743

H 2.33473 1.27971 4.06806

C 2.621601 .117671 .85393

H 3.506681 .716161 .67399

C $0.67730-0.145521 .44263$

C -0.39657 -0.49276 0.74076

C -0.39660 -0.49256-0.74062

C $0.67714-0.14497-1.44249$

C $0.846670 .00565-2.94112$

H 1.12426 -0.97957 -3.35459

C $2.045790 .92618-3.04752$

C $2.621921 .11730-1.85407$

H $2.334671 .27962-4.06816$ 


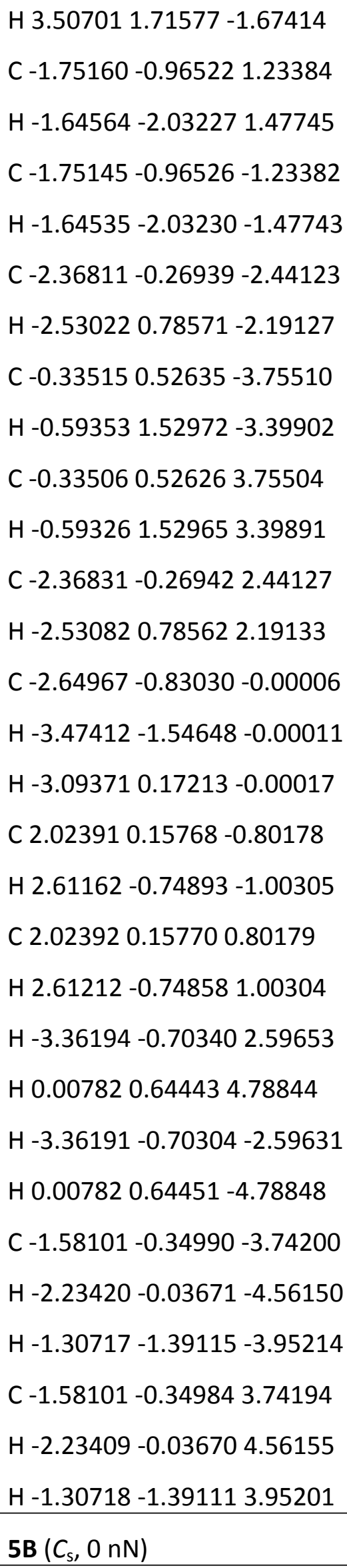




$$
\begin{aligned}
& E=-814.69160243 \\
& H=-814.27324674 \\
& G=-814.33047354 \\
& n_{\text {imag }}=0
\end{aligned}
$$

C 0.900190 .000022 .77811

H 1.12047 -1.07844 2.80543

C 2.224180 .733362 .81997

H 2.904000 .659933 .66121

C 2.480601 .401511 .69775

H 3.388701 .960911 .50663

C 0.417270 .353901 .40226

C -0.52090 -0.286620.72630

C $-0.52090-0.28662-0.72630$

C $0.417270 .35390-1.40226$

C $0.900190 .00002-2.77811$

H $1.12047-1.07844-2.80543$

C $2.224180 .73336-2.81997$

C $2.480601 .40151-1.69775$

H 2.90400 0.65992 -3.66121

H $3.388701 .96090-1.50663$

C -1.50748 -1.30010 1.23307

H -0.99077 -2.25226 1.42662

C - $-1.50748-1.30010-1.23307$

H -0.99077 -2.25226-1.42662

C - $2.19220-0.84733-2.52542$

H -2.63473 $0.14031-2.35240$

C - $0.165350 .23746-3.85260$

H -0.56719 1.25120 -3.75524

C - 0.165350 .237463 .85260

H -0.56719 1.251203 .75524 


$$
\begin{aligned}
& \text { C - } 2.19220-0.847332 .52542 \\
& \text { H -2.63473 } 0.140312 .35240 \\
& \text { C - }-2.42642-1.481880 .00000 \\
& \text { H - } 2.95573-2.436610 .00000 \\
& \text { H -3.18112 -0.68616 } 0.00000 \\
& \text { C } 1.269821 .43536-0.78808 \\
& \text { H } 0.777712 .38444-1.03480
\end{aligned}
$$

5B $\left(C_{\mathrm{s}}, 2 \mathrm{nN}\right)$

$E=-814.67426295$

$H=-814.25742595$

$G=-814.31444971$

$\mathrm{n}_{\mathrm{imag}}=0$

C $0.83648-0.000732 .89053$

H 1.01398 -1.07259 3.06885

C 2.147980 .728423 .08720

H 2.570060 .796174 .12134

C 2.607291 .231841 .93175

H 3.535601 .780571 .82357 


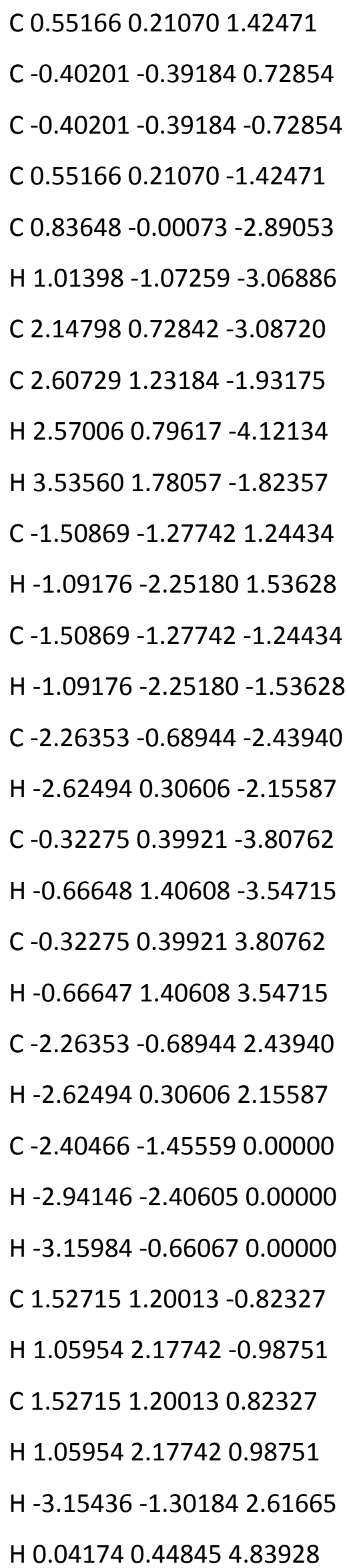




$$
\begin{aligned}
& \text { H -3.15436 -1.30184 -2.61665 } \\
& \text { H } 0.041740 .44845-4.83928 \\
& \text { C -1.49175 -0.58992 -3.75903 } \\
& \text { H -2.20417 -0.31694 -4.54313 } \\
& \text { H -1.11653 -1.58511 -4.02833 } \\
& \text { C -1.49175 -0.58992 } 3.75903 \\
& \text { H -2.20417 -0.31694 } 4.54313 \\
& \text { H -1.11654 -1.585114.02833 } \\
& \text { 5'A }\left(C_{\mathrm{s}}, 0 \mathrm{nN}\right) \\
& E=-814.67999218 \\
& H=-814.26092359 \\
& G=-814.31848839 \\
& \mathrm{n}_{\text {imag }}=0
\end{aligned}
$$

C 0.853410 .000172 .66551

H 1.046271 .065852 .48862

C $2.20300-0.675822 .67250$

H $2.94221-0.487353 .44291$

C $2.39463-1.451801 .61151$

H 3.30648 -1.99915 1.40404

C $0.23564-0.554751 .40180$

C $-0.79026-0.035200 .73617$

C - $0.79026-0.03520-0.73617$

C $0.23564-0.55475-1.40180$

C $0.853410 .00017-2.66551$

H $1.046271 .06585-2.48862$

C $2.20300-0.67582-2.67250$

C $2.39463-1.45180-1.61151$

H 2.94221 -0.48735 -3.44291

H 3.30648 -1.99915 -1.40404

C - 1.801850 .980181 .20869 


$$
\begin{aligned}
& \text { H -2.760320.44171 } 1.27592 \\
& \text { C -1.80185 0.98018-1.20869 } \\
& \text { H -2.76032 } 0.44171-1.27592 \\
& \text { C - } 1.913761 .928180 .00000 \\
& \text { H - } 2.836022 .513960 .00000 \\
& \text { H -1.06823 2.626280.00000 } \\
& \text { C } 1.13713-1.59704-0.78744 \\
& \text { H } 0.71843-2.57499-1.05269 \\
& \text { C } 1.13713-1.597040 .78744 \\
& \text { H } 0.71843 \text {-2.57499 } 1.05269 \\
& \text { C }-0.01614-0.06615-3.91817 \\
& \text { H -0.11607 -1.10265 -4.25209 } \\
& \text { H } 0.497250 .47142-4.72164 \\
& \text { C -0.01614-0.06615 } 3.91817 \\
& \text { H -0.11607 -1.10265 4.25209 } \\
& \text { H } 0.497250 .471424 .72164 \\
& \text { C - } 1.558581 .59699-2.58688 \\
& \text { H -0.68706 2.25811 -2.56328 } \\
& \text { H -2.41154 2.24079 -2.82055 } \\
& \text { C - } 1.422810 .53815-3.69831 \\
& \text { H -1.76329 } 0.96618-4.64421 \\
& \text { H -2.12414 -0.26978 -3.46892 } \\
& \text { C - } 1.558581 .596992 .58688 \\
& \text { H -2.41154 2.24079 } 2.82055 \\
& \text { H -0.68706 2.258112.56328 } \\
& \text { C - } 1.422810 .538153 .69831 \\
& \text { H -2.12414 -0.26978 } 3.46892 \\
& \text { H - } 1.763290 .966184 .64421 \\
& \text { 5'A }\left(C_{\mathrm{s}}, 2 \mathrm{nN}\right) \\
& E=-814.6566891 \\
& H=-814.23950953
\end{aligned}
$$




$$
\begin{aligned}
& G=-814.2967798 \\
& n_{\text {imag }}=0
\end{aligned}
$$

C 0.770650 .005972 .87342

H 0.865351 .094352 .99122

C $2.15446-0.563613 .09790$

H $2.57080-0.569604 .13683$

C $2.69757-0.989341 .94764$

H 3.69619 -1.39931 1.85179

C $0.49822-0.317171 .42116$

C - $0.59414-0.007130 .73233$

C -0.59409-0.00718 -0.73252

C $0.49796-0.31857-1.42127$

C $0.770690 .00349-2.87377$

H $0.868361 .09156-2.99188$

C $2.15283-0.56994-3.09871$

C $2.69594-0.99536-1.94836$

H $2.56829-0.57822-4.13797$

H $3.69369-1.40750$-1.85281

C -1.87971 0.618331 .22038

H -2.60707 -0.19616 1.35931

C - $1.878940 .61970-1.22072$

H -2.60673 -0.19412-1.36135

C -2.32998 1.441310 .00015

H -3.40229 $1.64986-0.00010$

H -1.802012.402930.00091

C $1.63816-1.11792-0.82676$

H $1.33507-2.15929-0.97679$

C $1.63899-1.116060 .82725$

H 1.33756 -2.15753 0.97986

C $-0.37369-0.44239-3.78747$ 


$$
\begin{aligned}
& \text { H -0.71329 -1.42910 -3.46105 } \\
& \text { H -0.01231 -0.56391 -4.81328 } \\
& \text { C }-0.37230-0.443003 .78735 \\
& \text { H -0.70911 -1.43066 } 3.46089 \\
& \text { H -0.01050 -0.56356 4.81312 } \\
& \text { C -1.77299 } 1.39755-2.52697 \\
& \text { H -0.97288 2.13781 -2.42310 } \\
& \text { H -2.69251 } 1.97457-2.66956 \\
& \text { C -1.55216 0.55617 -3.79330 } \\
& \text { H -1.40989 } 1.25748 \text {-4.62124 } \\
& \text { H -2.47291 } 0.01023-4.02043 \\
& \text { C -1.77562 } 1.394622 .52773 \\
& \text { H -2.69632 } 1.969672 .67067 \\
& \text { H -0.97694 2.13660 } 2.42513 \\
& \text { C -1.55356 0.55225 } 3.79324 \\
& \text { H -2.472980.00354 } 4.01911 \\
& \text { H -1.41384 1.25304 4.62204 }
\end{aligned}
$$$$
\text { 5'B }\left(C_{s}, 0 \mathrm{nN}\right)
$$$$
E=-814.70172803
$$$$
H=-814.2830518
$$$$
G=-814.3401009
$$$$
\mathrm{n}_{\text {imag }}=0
$$

C 0.829690 .002772 .87637

H 0.957040 .904253 .49804

C $2.18183-0.678482 .74383$

H 2.60713 -1.28316 3.53656

C $2.77753-0.400401 .58937$

H $3.76418-0.741001 .29974$

C 0.601730 .378761 .43308

C - 0.514680 .258160 .73271 


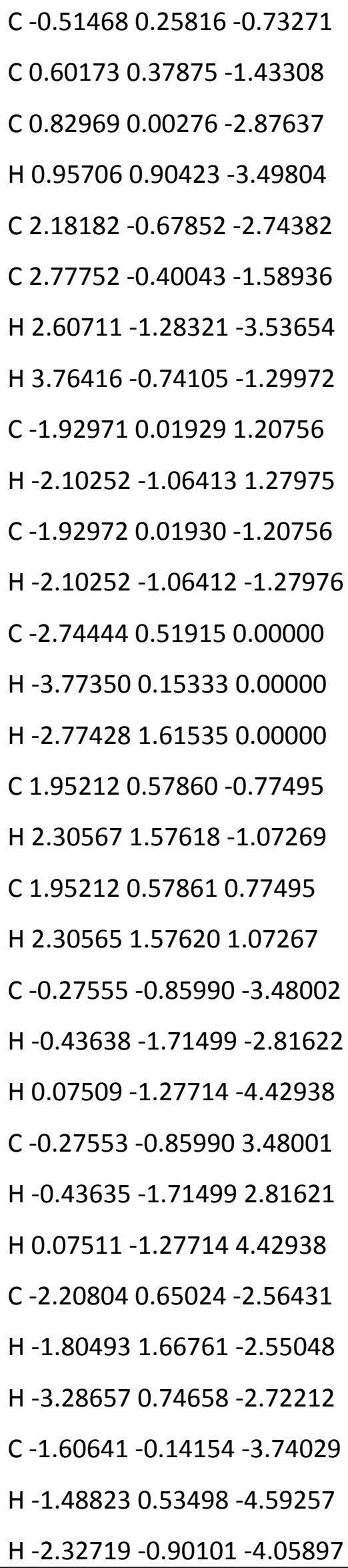




$$
\begin{aligned}
& C-2.208050 .650222 .56431 \\
& H-3.286570 .746552 .72212 \\
& \text { H -1.80494 } 1.667602 .55049 \\
& \text { C -1.60640 }-0.141563 .74029 \\
& \text { H - } 2.32718-0.901044 .05896 \\
& \text { H - } 1.488240 .534954 .59258
\end{aligned}
$$$$
\text { 5'B }\left(C_{\mathrm{s}}, 2 \mathrm{nN}\right)
$$$$
E=-814.68135752
$$$$
H=-814.26411848
$$$$
G=-814.32087129
$$$$
\mathrm{n}_{\text {imag }}=0
$$

C 0.783580 .003852 .93516 H 0.913161 .005053 .37996

C $2.10083-0.738173 .05618$

H 2.42855 -1.05550 4.07712

C $2.73118-0.808771 .87417$

H 3.70576 -1.25484 1.71457

C 0.640190 .128171 .43611

C - 0.481760 .146380 .73304

C - $0.481760 .14637-0.73307$

C $0.640180 .12802-1.43613$

C $0.783590 .00357-2.93517$

H $0.913501 .00471-3.38002$

C $2.10061-0.73887-3.05605$

C $2.73097-0.80937-1.87403$

H 2.42826 -1.05648 -4.07694

H $3.70544-1.25565$-1.71434

C -1.91353 0.22029 1.21742

H - $2.29968-0.801161 .34519$

C - $1.913510 .22045-1.21748$ 


$$
\begin{aligned}
& \text { H -2.29973 -0.80095 -1.34543 } \\
& \text { C - } 2.623600 .835340 .00000 \\
& \text { H -3.70048 } 0.65326-0.00002 \\
& \text { H -2.46756 } 1.921170 .00008 \\
& \text { C } 2.020650 .06346-0.80706 \\
& \text { H } 2.470721 .04461 \text {-1.01203 } \\
& \text { C } 2.020670 .063670 .80702 \\
& \text { H } 2.470601 .044941 .01172 \\
& \text { C -0.39764-0.68314 -3.62017 } \\
& \text { H -0.63307 -1.58865 -3.05272 } \\
& \text { H -0.09161 -1.02054 -4.61559 } \\
& \text { C -0.39745 -0.68321 } 3.62014 \\
& \text { H -0.63259 -1.58879 } 3.05267 \\
& \text { H -0.09134 -1.02053 4.61555 } \\
& \text { C -2.07397 } 0.98071-2.52793 \\
& \text { H -1.48531 } 1.90120-2.45464 \\
& \text { H -3.11585 } 1.29289-2.65019 \\
& \text { C -1.65144 } 0.18604-3.77082 \\
& \text { H -1.50405 } 0.88953-4.59643 \\
& \text { H -2.47583 -0.46657-4.07512 } \\
& \text { C - } 2.074150 .980342 .52798 \\
& \text { H -3.11610 } 1.292302 .65025 \\
& \text { H -1.48567 } 1.900952 .45484 \\
& \text { C -1.651510.18559 } 3.77079 \\
& \text { H -2.47574 -0.46729 4.07493 } \\
& \text { H -1.50439 0.88901 4.59650 }
\end{aligned}
$$

